\title{
Hydrodynamic Analysis and Optimization of a Hinged Type Wave Energy Converter
}

\author{
by \\ (C) Yuzhu Li
}

A Thesis submitted to the School of Graduate Studies in partial fulfillment of the requirements for the degree of

\author{
Master of Engineering
}

Faculty of Engineering and Applied Science

Memorial University of Newfoundland

May 2016

St. John's

Newfoundland 


\section{Abstract}

SeaWEED(Sea Wave Energy Extraction Device) is a multi-body floating wave energy converter (WEC) with hinged joints developed by Grey Island Energy Inc.(GIE) in Canada. Initial conceptual studies have been carried out to evaluate the performance of the first generation device by testing an 1:16 scale model in a wave basin. The experimental results were compared with the numerical solutions. Based on the experimental studies, improvements were made and a second generation model with a new geometry of the hull and a new connection structure was developed. This thesis is mainly focused on the numerical analysis and optimization of the second generation SeaWEED model. In the numerical studies, the hydraulic power take-off (PTO) system was simulated by a linear spring damper system coupled with the motion of the hinged bodies. The vertical hinge motion was computed at a series of wave periods using WAMIT. Optimization was focused on the PTO damping and the geometrical parameters in terms of the draft and the length of the truss structure between hinged bodies by using the response surface method. The optimization was conducted in regular waves and in irregular sea states. An optimal combination of length, draft and PTO damping was recommended for an intended operation location. 


\section{Acknowledgements}

I would like to express my special thanks of gratitude to my supervisor Dr. Heather Peng, who has patiently supported and supervised me during the master's program: Your kindness, encouragement, insight and dedication continue inspiring and motivating me in my education and research work. You always make me feel warm while studying abroad.

I would like to thank Dr. Wei Qiu, for his professional and technical teaching in my master's course learning. He helped me establish the theoretical knowledge and programming skills in numerical methodologies of hydrodynamics and trained me to become more professional in official communicating and academic writing. Thanks to Dr. Qiu for introducing the research topic on wave energy conversion to me and providing me the precious opportunity of an internship in ABS. Dr. Qiu and Dr. Peng are not only supervisors in my academic career but also life mentors who show me a perfect example of being dedicated and virtuous, having integrity and staying humble.

A special thanks goes to Brian Lundrigan, one of the founders of Grey Island Energy Incorporation, who came up with the great idea of SeaWEED wave energy converter. Without his ideas and fundamental studies on SeaWEED, I could not have the opportunity to work with the interesting topic regarding renewable energy. Brian explained me many of his great and innovative ideas within different fields, which are always a 
reminder for me to keep fresh, curious and creative in my life and research career. Furthermore I am grateful to my colleagues, especially my senior fellows Junshi Wang and Quan Zhou, who provided help to me through many difficult problems in my research experience. Thanks to all the other colleagues in our group; we studied together, worked together; and I am glad to see that we all made big progress during the study in the Advanced Marine Hydrodynamics Laboratory.

I would like to give a special thanks to Yuqi Ge: No matter how late I worked in the office on campus, you always came to pick me up and keep me safe on the way home. I owe huge thanks to my parents, who are the enlightenment to me and always give me the best thing that they can offer. They taught me to keep kind-hearted and optimistic, passionate and perseverant; these qualities I learned from them keep me forging ahead in my life.

Last but not least, I would like to express my appreciation to the NSERC CREATE Training Program for the financial support for my master's research program. 


\section{Table of Contents}

Abstract $\quad$ ii

Acknowledgments $\quad$ iii

Table of Contents vii

List of Tables viii

List of Figures $\quad$ xii

Nomenclature $\quad$ xii

1 Introduction 1

1.1 A Review of Wave Energy Conversion . . . . . . . . . . . . . . . . 1

1.1.1 Historical Perspective . . . . . . . . . . . . . . . . 1

1.1.2 Classification of Devices . . . . . . . . . . . . . 3

1.1.2.1 Oscillating Water Columns ........... 3

1.1.2.2 Oscillating Bodies ............. 5

1.1.2.3 Overtopping Devices . . . . . . . . . . . 7

1.2 A Review of Simulation-Based Design Methods . . . . . . . . . . 7

1.2.1 Optimization Objectives and Constraints . . . . . . . . . 9

1.2.2 Surrogate Modeling Methodology for Simulation-Based Design 10 
1.2.2.1 Design of Sampling Plans . . . . . . . . . . . . 11

1.2.2.2 The Choice of a Surrogate Model . . . . . . . . . . 14

1.2.2.3 Search the Optima Based on the Surrogate Model . . 16

1.3 Thesis Overview . . . . . . . . . . . . . . . . . . . . . . . . . . 17

2 SeaWEED Wave Energy Converter 20

2.1 An Introduction of SeaWEED . . . . . . . . . . . . . . . 20

2.2 Power Take-Off Mechanism of SeaWEED . . . . . . . . . . . . . . . 23

2.3 Competitive Advantages $\ldots \ldots \ldots \ldots \ldots$

3 Theoretical Background and Mathematical Formulation $\quad 27$

3.1 Motion of Hinged Bodies in Waves _. . . . . . . . . . . 27

3.1.1 Two General Approaches for Multi-Body System . . . . . . . . 27

3.1.2 Newman's Approach for Hinged Arrays . . . . . . . . . . . 28

3.1 .3 Mass Matrix of a Hinged Array . . . . . . . . . . . . . . . 31

3.1.4 Boundary Value Problem of Hinged Arrays . . . . . . . . . 33

3.2 Numerical Modeling of Power Take-Off system . . . . . . . . . . . . 39

3.2.1 Power Absorption in Regular Waves . . . . . . . . . . . . . . 39

3.2.2 Power Absorption in Irregular Sea States . . . . . . . . . . . 40

3.2.3 Power Absorption at a Certain Location . . . . . . . . . . . 42

3.2 .4 Power Absorption of SeaWEED . . . . . . . . . . . . . . 42

4 Model Test and Frequency Domain Simulation 45

4.1 Model Test of the First Generation Seaweed . . . . . . . . . 45

4.1 .1 Overview of the Model Test . . . . . . . . . . . . . . . 45

4.1.2 Comparison between Experimental and Numerical Results . . 47

4.1 .3 Improvements . . . . . . . . . . . . . . . . . . . . . . 48

4.2 Frequency Domain Simulation on the Second Generation of SeaWEED 50 
4.2 .1 Convergence Study . . . . . . . . . . . . . . . . . . . . . 52

4.2.2 Motion of the Second Generation of SeaWEED in Waves . . 54

5 Numerical Optimization of the Second Generation SeaWEED Model 58

5.1 Design of the Optimization Experiments . . . . . . . . . . 58

5.2 Numerical Optimization in Regular Waves . . . . . . . . . . . . . 60

5.2 .1 Optimization of PTO Parameters . . . . . . . . . . . . . 60

5.2.2 Optimization of the Geometrical Parameters . . . . . . . . . 62

5.3 Numerical Optimization at an Intended Site . . . . . . . . . 68

5.4 Comparison of the Power Absorption Performance . . . . . . . . . 74

5.4.1 Comparison between the Original and Optimized SeaWEED Models . . . . . . . . . . . . . . . . . . . . . . . 74

5.4.2 Comparison between the First and the Second Generation of SeaWEED models . . . . . . . . . . . . . 76

6 Conclusions and Limitations $\quad 78$

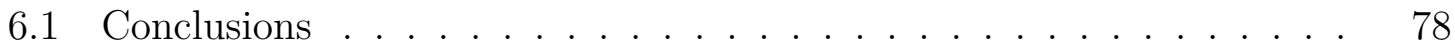

6.2 Limitations . . . . . . . . . . . . . . . . . . . . . . 80 


\section{List of Tables}

4.1 Parameters of the first generation SeaWEED model. . . . . . . . . . . 46

4.2 Initially designed principal parameters of SeaWEED in full scale. . . 51

4.3 Lengths of three segments for the initially designed SeaWEED, from stern to bow. . . . . . . . . . . . . . . . . . . . 52

4.4 Convergence study on the panel numbers. . . . . . . . . . . . 53

5.1 Overview of the optimization design in regular waves. . . . . . . . 63

5.2 Optimization solutions at each wave period. . . . . . . . . 68

5.3 Optimization overview for an intended site. . . . . . . . . . . 71

5.4 Verification studies on the polynomial model. . . . . . . . . . . 73

5.5 Optimal solution, predicted annual power and computed annual power absorption. ............................. 74

5.6 Parameters of the models been compared. . . . . . . . . . . . . 75 


\section{List of Figures}

1.1 Classification of wave energy converters (Falcão, 2010). . . . . . . . . 4

1.2 Pico Plant Azores (Falcão, 2000). . . . . . . . . . . . . . . . . 4

1.3 Prototype of Spar Buoy (NAREC, 2012). . . . . . . . . . . . . 5

1.4 A 3D simulation of the $F O^{3}$ wave energy converter (Taghipour et al.,

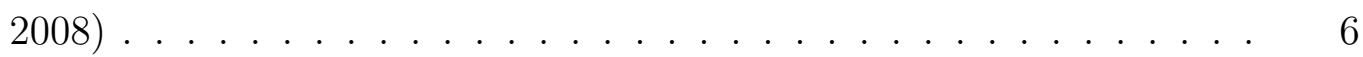

1.5 Prototype of Pelamis $($ Yemm, 2008). . . . . . . . . . . . . . . 7

1.6 Wave overtopping of the Wave Dragon prototype leads to power generation(Hansens et al., 2000). . . . . . . . . . . . . . 8

2.1 The first generation of SeaWEED system. . . . . . . . . . . . 20

2.2 Illustration of the PTO and the complete first generation SeaWEED system. . . . . . . . . . . . . . . . . . . . 21

2.3 Fully assembled second generation of SeaWEED system. . . . . . . . 22

2.4 Four modular sections of SeaWEED. . . . . . . . . . . . . . . 22

2.5 SeaWEED power take-off system. . . . . . . . . . . . 23

2.6 Profile views of the SeaWEED hydraulic power take-off system. . . . 24

2.7 Schematic of Pelamis selectable-tunable joint response (Yemm et al.,

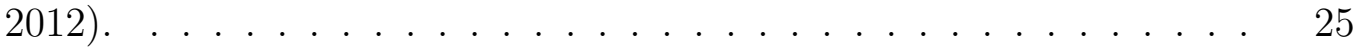

2.8 Pelamis P2 hydraulic PTO joint (Yemm et al., 2012). . . . . . . . . 25 
3.1 An array of hinged modules. . . . . . . . . . . . . . . . . . . 29

3.2 Illustration of general hinge modes. . . . . . . . . . . . . . . . . . . . 29

3.3 Illustration of heave and pitch modes. . . . . . . . . . . . . . . 30

3.4 General tent function. . . . . . . . . . . . . . . . . . 31

3.5 Hinge modes of SeaWEED illustrated in angular motions. . . . . . . . 43

4.1 The 1:16 model of the first generation SeaWEED in wave tank. . . . 46

4.2 The 1:16 model of the first generation SeaWEED in waves. . . . . . . 47

4.3 Comparison of the absorbed power between model test and numerical simulation for the first generation of SeaWEED model at a wave height of 3 meters. . . . . . . . . . . . . . . . . . . . . . 49

4.4 Comparison of the absorbed power between model test and numerical simulation for the first generation of SeaWEED model at a wave height of 4 meters. . . . . . . . . . . . . . . . .

4.5 Comparison of the absorbed power between model test and numerical simulation for the first generation of SeaWEED model at a wave height of 5 meters. . . . . . . . . . . . . . . . . 50

4.6 Dimension of Initial Designed SeaWEED wave energy converter. . . . 51

4.7 Convergence study on the panel numbers to model SeaWEED in WAMIT. 53

4.8 Free hinge motion of SeaWEED . . . . . . . . . . . . . . 55

4.9 Restrained hinge motion of SeaWEED with a $7 \times 10^{5} \mathrm{~N} \cdot \mathrm{s} / \mathrm{m}$ PTO damping. . . . . . . . . . . . . . . . . . . . . . 55

4.10 Restrained hinge motion of SeaWEED with a $7 \times 10^{5} \mathrm{~N} \cdot \mathrm{s} / \mathrm{m}$ PTO damping within 5.5 to 12 seconds. . . . . . . . . . . . . . . 56

4.11 Power capture width of the primarily designed second generation of SeaWEED with a $7 \times 10^{5} \mathrm{~N} \cdot \mathrm{s} / \mathrm{m}$ PTO damping within 5.5 to 12 seconds. 57 
5.1 Response curve of the power capture(absorption) width integration with different damping values of the SeaWEED model with a 30-meter length of intermediate truss and a 1.5-meter draft. . . . . . . . . . .

5.2 Power capture width comparison between original designed damping and optimal damping. . . . . . . . . . . . . . . .

5.3 RAO curves at two hinge joints of the initial model with an optimal PTO damping. . . . . . . . . . . . . . . . . .

5.4 Response surface of the power capture width integration in regular waves with a wave period at 6 seconds. . . . . . . . . . . .

5.5 Response surface of the power capture width integration in regular waves with a wave period at 7 seconds. . . . . . . . . . . . .

5.6 Response surface of the power capture width integration in regular waves with a wave period at 8 seconds. . . . . . . . . . . .

5.7 Response surface of the power capture width integration in regular waves with a wave period at 9 seconds. . . . . . . . . . . . .

5.8 Response surface of the power capture width integration in regular waves with a wave period at 10 seconds. . . . . . . . . . . .

5.9 Response surface of the power capture width integration in regular waves with a wave period at 11 seconds. . . . . . . . . . . . . . 67

5.10 Response surface of the power capture width integration in regular waves with a wave period at 12 seconds. . . . . . . . . . . . . . . . 67

5.11 Optimal truss length at each wave period. . . . . . . . . . . 69

5.12 Optimal draft at each wave period. . . . . . . . . . . . . 69

5.13 Optimal PTO damping at each wave period. . . . . . . . . . . . 70 
5.14 Wave climate table for a sea location off Cork Harbour in Ireland; the datasheet comprises wave data collected from the SmartBay buoy moored in Cork Harbour (SmartBay, 2015). . . . . . . . . . . . . . 71

5.15 Response surface of the annual power absorption at an intended location. 73

5.16 Comparison of the power capture widths between three of the second generation SeaWEED models. . . . . . . . . . . . . . . . 75

5.17 Comparison of the absorbed power between three of the second generation SeaWEED models at a 3-meter wave height. . . . . . . . . . 76

5.18 Comparison of the absorbed power between the first and the second generation SeaWEED models at a 3 -meter wave height. . . . . . . . . 


\title{
Nomenclature
}

\author{
$a_{i j} \quad$ Added-mass term \\ $A_{i} \quad$ Amplitude of incident monochromatic waves \\ $b_{i j} \quad$ hydrodynamic damping term \\ $c_{i j} \quad$ Restoring coefficient term \\ $c_{g} \quad$ Group velocity in deep water \\ $C_{\text {width }} \quad$ Power capture width: width of the wave front containing the \\ same available power as the useful power captured by the device \\ in the same wave climate. \\ d Translational PTO damping \\ $d_{\theta} \quad$ Rotary PTO damping \\ $f(x) \quad$ Shape function of a hinged array \\ $F_{i} \quad$ Hydrodynamic force acting on the body \\ $F_{R_{i}} \quad$ Radiation force of i-th mode
}




\begin{tabular}{|c|c|}
\hline$F_{c_{i}}$ & Hydrostatic force of i-th mode \\
\hline$F_{P}$ & Force that related to power take-off \\
\hline$g$ & Gravity of Earth \\
\hline$h$ & Water depth \\
\hline$H$ & Incident wave height in regular waves \\
\hline$H_{s}$ & Significant wave height of irregular seas \\
\hline$k$ & Wavenumber \\
\hline$K$ & Infinite-depth wave number \\
\hline$L$ & Length of the interconnecting truss structure \\
\hline$m(x)$ & Mass distribution of the device along $\mathrm{x}$-axis \\
\hline$M_{i j}$ & Mass matrix of a hinged array \\
\hline$n_{j}$ & Normal component of displacement on the surface \\
\hline$n_{x}$ & Normal vector along the $\mathrm{x}$ direction \\
\hline$n_{y}$ & Normal vector along the y direction \\
\hline$n_{z}$ & Normal vector along the $\mathrm{z}$ direction \\
\hline$N$ & Total number of hinged bodies \\
\hline$p$ & Pressure field in the fluid domain \\
\hline
\end{tabular}




\begin{tabular}{|c|c|}
\hline$p_{a}$ & Atmosphere pressure \\
\hline$P_{a b s}$ & Average power absorption in irregular seas \\
\hline$P_{w}$ & $\begin{array}{l}\text { Wave power of incident wave that can be extracted per meter } \\
\text { of crest }\end{array}$ \\
\hline $\bar{P}$ & Time average power absorption in regular waves \\
\hline$S$ & The collection of boundary surfaces \\
\hline$S_{b}$ & Submerged body surface \\
\hline$S_{A}$ & Wave spectrum \\
\hline$S_{x i}$ & Response spectrum \\
\hline$T$ & Draft of the device \\
\hline$T_{p}$ & Peak period of irregular seas \\
\hline$X_{i}$ & Wave exciting force \\
\hline$(x, y, z)$ & 3-dimensional coordinates in Cartesian system \\
\hline$\eta_{i}$ & Probability factor of a sea state \\
\hline$\gamma$ & Peakedness parameter for JONSWAP spectrum \\
\hline$\Phi$ & Velocity potential in time domain \\
\hline$\varphi$ & Complex velocity potential related to $\Phi$ \\
\hline
\end{tabular}




$\begin{array}{ll}\varphi_{D} & \text { Diffraction wave velocity potential in } \vec{x} \\ \varphi_{I} & \text { Incident wave velocity potential in } \vec{x} \\ \varphi_{R} & \text { Radiation wave velocity potential in } \vec{x} \\ \omega & \text { Wave frequency } \\ \omega_{p} & \text { Modal frequency corresponding to the highest peak of the spec- } \\ & \text { trum } \\ \xi_{i} & \text { Amplitude of response motion of the corresponding mode } \\ & \text { Density of fluid }\end{array}$




\section{Chapter 1}

\section{Introduction}

\subsection{A Review of Wave Energy Conversion}

\subsubsection{Historical Perspective}

During the past decades, increasing global energy consumption has accompanied increasing awareness of growing environmental problems and resource crises. Thus, many scientists and researchers have turned their eyes to the extraction of green and renewable energies, such as solar, wind and ocean energy. Ocean wave energy is a concentrated form of wind energy that is originally transferred from solar energy.

The wave power density is measured by power per length of wave crest, $[\mathrm{W} / \mathrm{m}]$; while wind and solar energy are measured in the unit of $\left[\mathrm{W} / \mathrm{m}^{2}\right]$ (Waters, 2008). Compared with solar and wind energy, ocean waves contain a higher intensity of power. For example in Sweden with a mild wave climate, the average solar radiation in Sweden is $0.12 \mathrm{~kW} / \mathrm{m}^{2}$, the average wind power flow density is $0.23 \mathrm{~kW} / \mathrm{m}^{2}$ near the coasts of Sweden (Bergström, 2007), and the average wave power is approximately $5.2 \mathrm{~kW} / \mathrm{m}^{2}$ on the west coast (Waters et al., 2009). Global wave power worldwide was estimated to be in the order of $1 \mathrm{TW}$ by Falnes et al. (2002). The vast amount of wave power 
on the ocean surface has led many to consider how to make use of it.

Although initial research on wave energy dates back to 1799 (Ross, 1995), it was not until the oil crisis in the 1970s that the topic began drawing worldwide attention. (Cruz, 2007) mentioned that in the 1960s, pioneer work in wave energy conversion was conducted by Japanese navy who built a marker buoy that used waves to power its lamp. After the oil crisis in 1973, Salter (1974) proposed the use of wave power and presented a design concept of a cam-shaped oscillating wave energy converter (WEC), known today as the Salter's Duck. This became a landmark that spurred worldwide research in WECs. Budar and Falnes (1975) defined the damped oscillator system as a 'point absorber' as its horizontal length is much shorter than the wavelength. Evans (1976) reviewed the theoretical principles of wave energy conversion and analytically derived the motion of the point absorber in waves.

Wave energy conversion is a hydrodynamic process with complex diffraction and radiation problems in which a system of mathematical hydrodynamic theories needed to be established. Wehausen and Laitone (1960) published a comprehensive review of Surface Waves, providing an important theoretical resource for the hydrodynamics of water waves. In the latter half of the 1970 s, research of wave energy conversion was mainly focus on wave theories and hydrodynamics (Falcão, 2010). Le Mehaute (1976) gave a general review of hydrodynamics and wave theories in his book. Newman (1977) presented an analytical system of marine hydrodynamic theories in terms of wave effects, motion of bodies in waves and hydrodynamic forces, based on Wehausen (1971)'s derivation of ship motion and hydrodynamic coefficients such as added-mass and damping. In the book of Mei (1989), theories of hydrodynamics, wave energy absorption and basic principles of wave energy extraction were discussed. Falnes (2002) developed a complete mathematical system for wave energy converters of oscillating bodies and the principles of optimum control in order to maximize the energy 
absorption of the device.

\subsubsection{Classification of Devices}

A variety of WEC designs have been proposed over the last two decades. Clément et al. (2002) gave a detailed review of the development and current status of wave energy conversion in a number of coastal nations in Europe including the United Kingdom, Ireland, Sweden, Denmark, Portugal, and Norway. Until 2015, there are more than one hundred projects of wave energy converters and more than one thousand patents worldwide (Day et al., 2015). Extensive numerical and experimental studies have been carried out to evaluate the performance of the WECs. Generally, the various designs of WECs can be classified into three types according to their working principles: oscillating water columns (OWCs), oscillating bodies, and wave overtopping devices. Falcão (2010) presented a well-known diagram of the classification of WECs, as shown in Figure 1.1 below.

\subsubsection{Oscillating Water Columns}

Oscillating water columns (OWCs) can be either fixed or floating. They have a partly submerged structure where an internal free surface exists in the inner chamber; air is trapped above the internal free surface. The oscillation of the free surface forces the air flow to propel an air turbine that drives the electrical generator. In Portugal, the shoreline of the OWC started construction in Pico of the Azores in 1986 (Falcão, 2000), which is a fixed structure built upon the shore, as shown in Figure 1.2. An example of a floating OWC is the Spar Buoy which consists of a longer submerged tail and a floater that heaves vertically in waves. As shown in Figure 1.3, a 1:16 scale model of Spar Buoy with a 1-meter diameter and a 3-meter draft was built and tested by the National Renewable Energy Centre (NAREC) in the UK. 

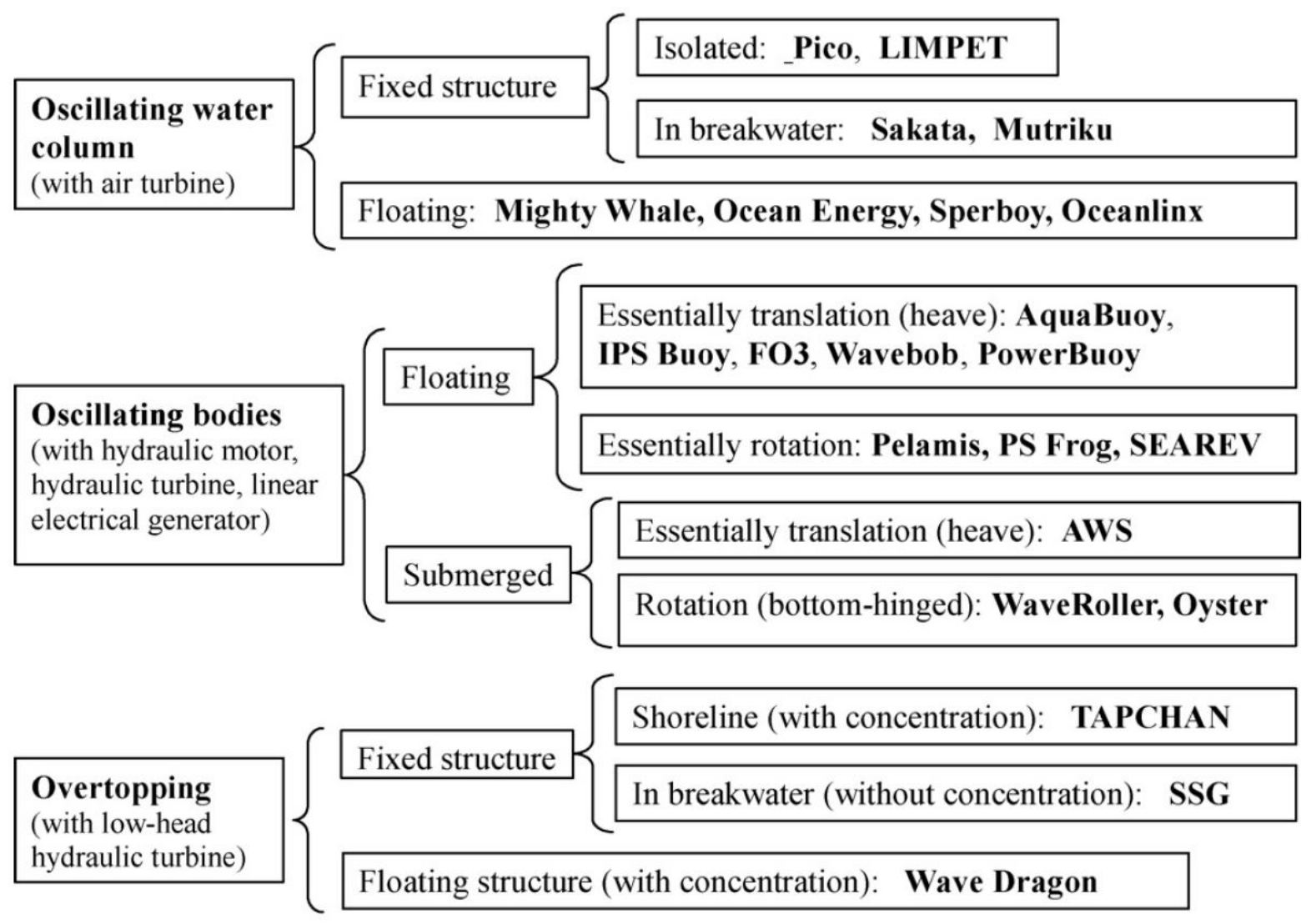

Figure 1.1: Classification of wave energy converters (Falcão, 2010).

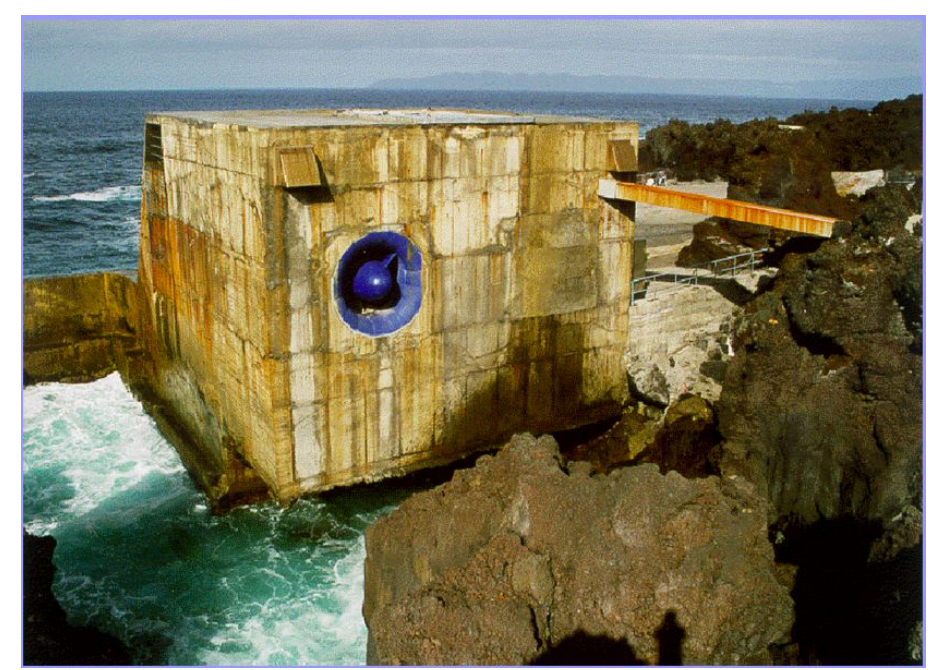

Figure 1.2: Pico Plant Azores (Falcão, 2000). 


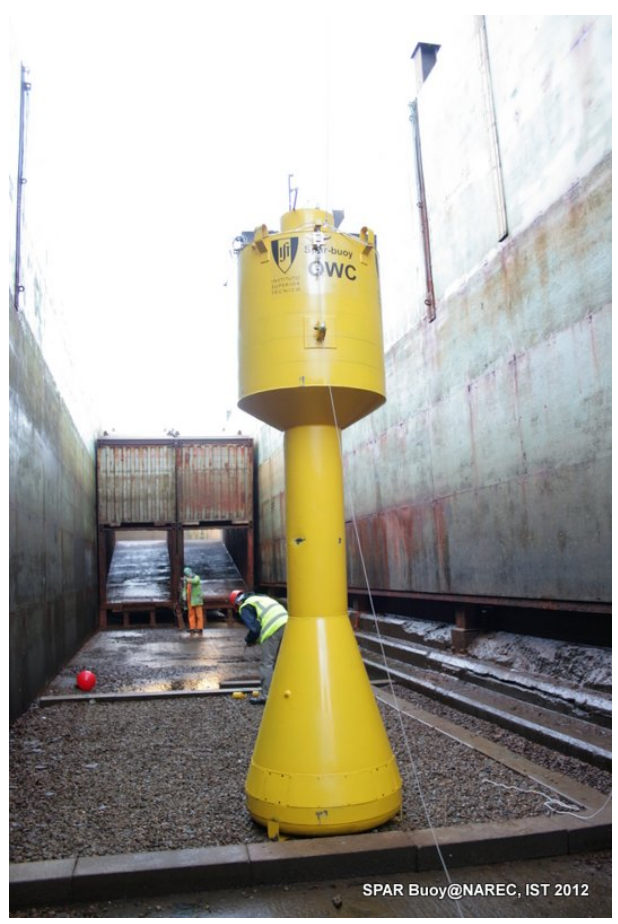

Figure 1.3: Prototype of Spar Buoy (NAREC, 2012).

\subsubsection{Oscillating Bodies}

Oscillating bodies are often based on the hydraulic power take-off system (PTO), which converts mechanical motion power into electrical power. This type of device can be a single-body that moves relative to the sea-bed or multi-bodies with relative motions between each body. The mechanical translational or angular motions in terms of heave or pitch drive the hydraulic PTO system to extract the power. Oscillating bodies can be further categorized as either floating devices or submerged devices. Floating devices include point absorbers and attenuators. Salter's Duck (1974), developed in the UK, is a typical point absorber oscillating body wave energy converter, which is still regarded as being one of the most efficient. The Wavebob, developed in Ireland by Weber et al. (2009), is an axisymmetric two-body heaving absorber. In Sweden, a two-body point absorber IPS buoy was invented by Noren (1981). A concept of point absorber WECs called $F O^{3}$ was developed by Fred. Olsen 
Ltd. (Taghipour et al., 2008). It consists of a number of heaving floaters attached to a floating platform. The vertical heave motions are converted into angular movements by the hydraulic system that drive the hydraulic motors. A 3D model simulation of the $\mathrm{FO}^{3}$ WEC is shown in Figure 1.4.

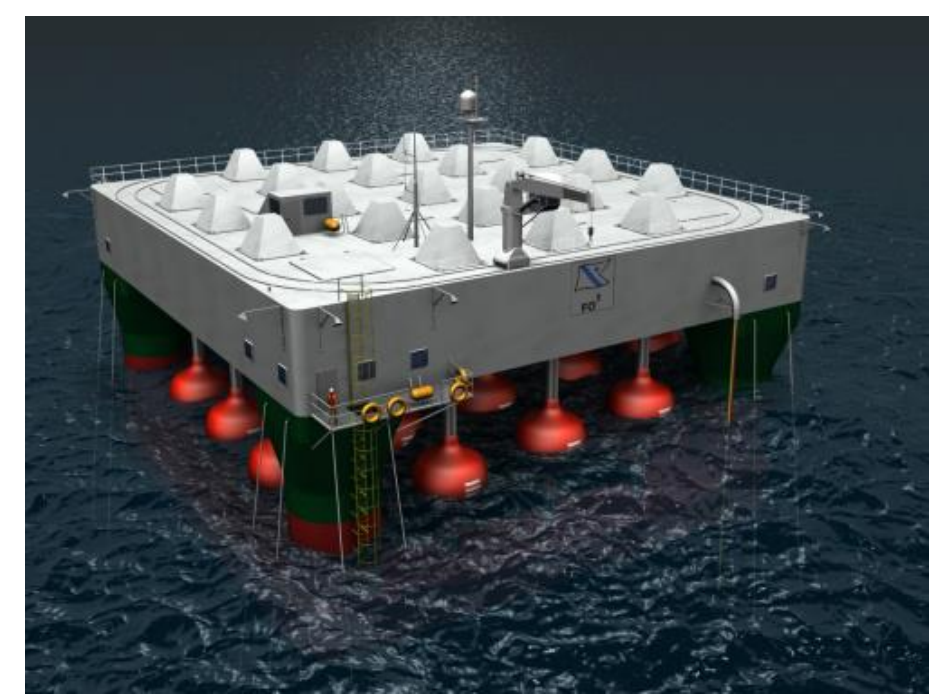

Figure 1.4: A 3D simulation of the $\mathrm{FO}^{3}$ wave energy converter (Taghipour et al., 2008)

An attenuator type WEC typically has a slender body and is installed parallel to the wave propagation direction. In Denmark, a half scale attenuator type WEC Wave Star was deployed by Bjerrum (2008), consisting of a number of floaters on movable arms. Another well-known example of an attenuator type oscillating body WEC is Pelamis, developed by Pizer et al. (2000)). In 2008, a wave farm consisting of $750 \mathrm{~kW}$ Pelamis devices was tested in northern Portugal. In 2010, another Pelamis wave farm was tested in Orkney, Scotland. Figure 1.5 shows a prototype of Pelamis by Yemm (2008). 


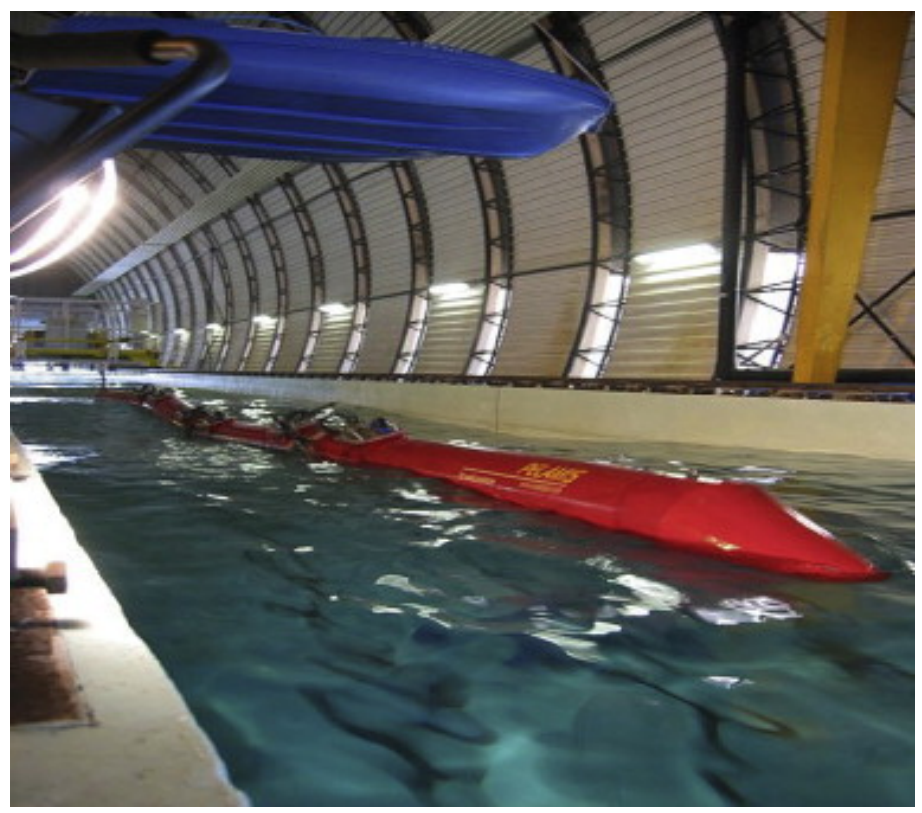

Figure 1.5: Prototype of Pelamis (Yemm, 2008).

\subsubsection{Overtopping Devices}

Overtopping devices capture wave power by converting potential energy into electrical energy. The water waves come into the reservoir above the sea level and return to the sea through conventional low head hydro-turbines. Wave Dragon (Hansens et al., 2000 ) is a well-known overtopping wave energy converter. A 1:4.5 scale prototype of Wave Dragon was deployed in Denmark in 2003, as shown in Figure 1.6. The Sea Slot-cone Generator (SSG) is another overtopping type WEC, consisting of several reservoirs (Margheritini et al., 2009).

\subsection{A Review of Simulation-Based Design Meth- ods}

The development of WECs can benefit from the advancement of simulation tools as the manufacture of prototypes requires much more time and cost. Due to the advance- 


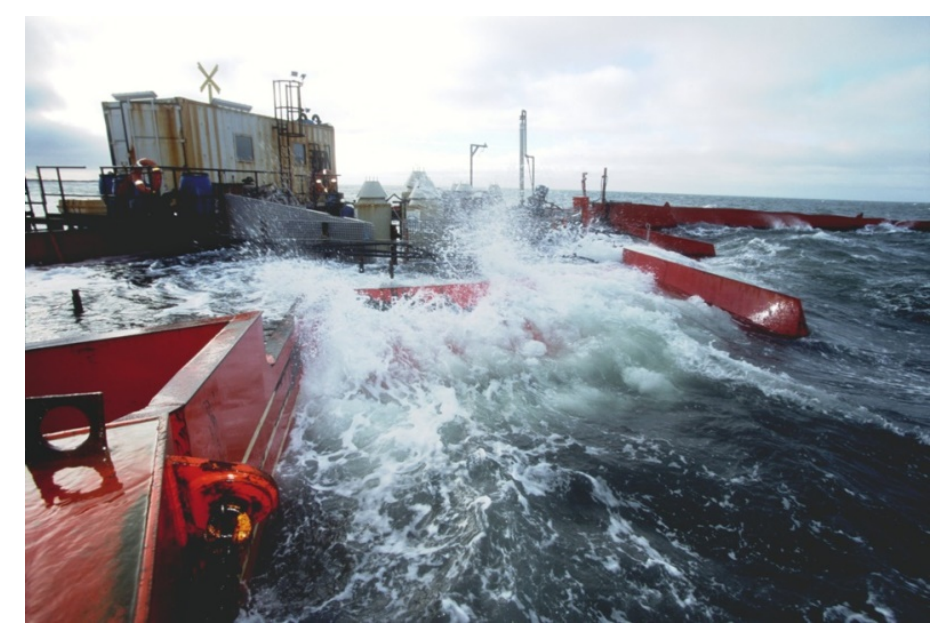

Figure 1.6: Wave overtopping of the Wave Dragon prototype leads to power generation(Hansens et al., 2000).

ment in computational power and tools, computer simulations have been increasingly applied to evaluate and optimize complex hydrodynamic problems. Compared to the design of physical experiments, simulation-based design (SBD) has many beneficial features, thus the design methodology is normally different from the design of a physical one (Jones and Johnson, 2009). For example, most of the simulation-based experiments are deterministic, meaning that they do not have the random errors from the physical world, i.e., running with the same input and constraints will result in the identical response; thus the replication, randomization and blockings are not applicable in the SBDs. In addition, an SBD is able to consider a large number of variables at the same time, which is not feasible in the real world.

Tahara et al. (2011) presented the general expression of simulation-based optimization problems. A $M$ dimensional real space with design variables $\mathbf{x}=\left(x_{1}, x_{2}, \ldots, x_{M}\right)^{T}$. The $N$ objectives of the optimization are $\mathbf{F}=\left(F_{1}, F_{2}, \ldots, F_{N}\right)^{T}$. The optimization 
problem is formulated as

$$
\begin{aligned}
& \min \text { or } \max \mathbf{f}(\mathbf{x}), \mathbf{x} \in X \subseteq \mathcal{R}^{M} \\
& \begin{cases}h_{j}(\mathbf{x})=0, & j=1, \ldots, p \\
g_{j}(\mathbf{x}) \leq 0, & j=1, \ldots, q \\
x_{i}^{l} \leq x_{i} \leq x_{i}^{u} & \end{cases}
\end{aligned}
$$

where $\mathbf{f}$ is a $\mathrm{N}$-dimensional vector of objective functions, $\mathbf{x}$ is a vector of design variables, $h_{j}$ and $g_{j}$ are the equality and inequality constraints to the variables respectively, and the superscripts $l$ and $u$ refer to the lower and upper bounds of the variables.

\subsubsection{Optimization Objectives and Constraints}

The objective of the design is to minimize or maximize the objective function or functions. For the optimization of WECs, the objective can be maximizing the yearly energy production while minimizing the production cost (Babarit et al., 2005).

For PTO optimization, it is usually a single-objective problem for which the maximum power absorption performance is the goal. For geometrical optimization, there is sometimes a compromise between the superior power absorption and economical costs. Thus it is usually a multi-objective problem, for which both the power absorption performance and the cost of building the device are taken in to consideration.

Kurniawan and Moan (2013) stated that for geometry design of a WEC, especially wave absorbers, the minimum of submerged surface area, submerged volume, as well as the surface curvature will lead to the lowest construction cost as well as construction difficulty. Babarit et al. (2005) conducted a geometric optimization of the SEAREV by focusing two objectives: to maximize the absorbed power at a given site and to minimize the total mass in order to minimize the cost. To simplify the multi-objective 
problem, some researchers consider two types of ratios as simple criteria for optimizing wave absorbers: the ratio of the submerged surface area to the absorbed power and the ratio of the reaction force to the absorbed power (French et al., 1996).

During optimization, constraints must be satisfied while achieving the objectives. For the optimization of WECs, there are several general constraints including the slamming constraints and force constraints. For example, for wave absorbers, Kurniawan and Moan (2013) constrained the displacement of the body, which was set as a ratio of the wave amplitude. For slender hinged-body WECs, the hinged joints normally have a restrained angle so that the angular motions of the bodies are constrained. Also, the generator normally has limited capacity that a upper bound of absorbed power will be set.

\subsubsection{Surrogate Modeling Methodology for Simulation-Based Design}

As it is mentioned before that simulation-based experiments have the merit of faster implementation that can largely reduce the time and expense of making physical prototypes. However, the complexity of the problem, long running time and high computation cost can become obstacles for simulation-based experiments. Meanwhile, unknown of gradients of the model often becomes another barrier. Kushner and Clark (2012) discussed an approach called stochastic counterpart (SC) method, which is known as the concept of 'surrogate models' or 'meta-models' approach. The idea of the surrogate modeling approach is to spread samples $\mathbf{x}$ among the design space of interest, based on which to construct a mathematical surrogate model $A(x)$ to represent the relation between factors and responses, which approximates the real objective function $F(x)$. Then, the optimization procedures are implemented by studying the surrogate model $A(x)$ with higher efficiency and lower expense. The model $A(x)$ is determined 
by minimizing the discrepancy between $A(x)$ and the objective function $F(x)$ over the sampling points $\mathbf{x}$. The major benefit of using surrogate models is that they are cheap and efficient.

However, surrogate modeling is heuristic; it does not ensure the exact correct answer for the original system. Rubinstein (1997) found that when the sample size $N$ goes to infinity, the surrogate model converges to the true model. Thus, one has to make compromise between the accuracy of the model and the efficiency in calculation, since an accurate model may need a larger sample size which results in longer time to compute. In general, using surrogate models requires additional computer simulation experiments to verify the results and performing physical experiments to validate the model and the optimized solutions (Johnson et al., 2008). Surrogate modeling approaches for the simulation-based experiments generally involve four steps:

- Design a sampling plan within the design space;

- Use a surrogate model to fit the relation between inputs and the response(s);

- Verification and validation of the surrogate model;

- Finding the optima based on the surrogate model.

\subsubsection{Design of Sampling Plans}

There are numerous approaches to design a sampling plan. A straightforward design approach is full factorial design (Ryan and Morgan, 2007). A full factorial design consists of two or more factors, each with discrete levels; and the experimental cases take on all possible combinations of these levels across all such factors. Full factorial design can take most information into consideration that the effect of each factor on the responses and the interaction between factors and responses can be more clearly 
studied. However, when the number of combinations is very high that the full factorial design is not logistically feasible, a more efficient and design needs to be conducted. Nowadays, for computer simulation experiments, a very popular and standard design approach is the space-filling design. Space-filling design is to spread the input samples over the design space as uniformly as possible Pronzato and Müller (2012). A distinctive feature of space-filling design is that, in the design space of interest, every sample point is unique; and the uniqueness of each sample point is kept even when the space dimension is curtailed. This feature makes the space-filling design approach comprehensive and compendious, since an additional sample point which contains repetitive feature provides no more requisite information.

Pronzato and Müller (2012) investigated the previous literature and pointed that when experiment is deterministic with no randomness, such as most of the simulationbased experiments, space-filling design is an appropriate choice; when the experiment is stochastic, like the real-world experiments, or some stochastic computer simulations containing randomness or noises, such as Discrete Event Simulation (DES) (Johnson et al., 2011), it is recommended to use response surface designs (RSM) such as central composite design (CCD), factorial design (FD), or optimal designs.

Under the category of space-filling design, a host of methods were proposed over the last several decades. Currently, one of the most popular methods is Latin hypercube design (LHD). The Latin hypercube sampling approaches was proposed by McKay et al. (1979), in which the sampling points are distributed far from each other, yielding the maximin distance and without replication in each projection. In a large number of literatures, it is often coupled with the use of Gaussian process (GP) models to fit the surrogate model surface.

In early 1980s, a uniform design (UD) approach is created and developed by Fang (1980) with the seeking of a uniform distribution of the sample points in the design 
space. Fang et al. (2000) discussed UD theory in detail and gave the instructions of the UD applications. The concrete steps of performing a UD is summarized by Fang and $\operatorname{Lin}(2003)$.

- Choose factors and experimental domain as well as determine suitable number of levels for each factor;

- Choose a suitable UD to accommodate the number of factors and levels;

- From the UD table, randomize the run order if necessary and conduct the experiments;

- Find a suitable model to fit the data. E.g., regression analysis, neural networks, wavelets, multivariate splines, etc.;

- Knowledge discovery from the built model. Optimize response if required.

Fang and Lin (2003) suggested that the UD can be followed by regression models for fitting the model surface when the relationship between the responses and factors is nonlinear or when the design levels are equal or more than two.

Islam and Lye (2009) performed a second-order computer experimental design to investigate the relationship between the thrust coefficient and the five parameters. They did a comparison between using two classical response surface designs in terms of central composite design (CCD), Box-Behnken designs (BBD) and a space-filling design UD with using a second-order centered quadratic regression model to fit the surface of response. It is showed in their paper that the UD performed the best in this certain problem with fewer runs than CCD and better accuracy among the three approaches. They discovered that when dealing with four or more factors and when the true model is highly nonlinear, uniform design gives an efficient and robust 
performance. The choice of the sample size of UD should be based on the seeking of minimal runs and the complexity of the model.

Recently, a new space-filling design approach called fast flexible filling (FFF) designs is introduced by Lekivetz and Jones (2014); it works for both rectangular and nonrectangular regions of design space. In their paper, they made a comparison between minimax design, uniform design and an orthogonal maximin Latin hypercube design (OLHD) (Joseph et al., 2008); it is showed that the FFF design used minimal inputs and gave out a model with high accuracy.

In fact, no one design type is generally better than the others (Johnson et al., 2011). The accuracy of the surrogate model is largely related to the sample size and the complexity of the objective function. To choose an appropriate design, one needs to consider the feature of the problem to be solved and also the coupled model fitting methods.

\subsubsection{The Choice of a Surrogate Model}

Accompanying with the design of a sampling plan, a surrogate model needs to be chosen to fit the relationship between the responses and the variable samplings over the design space. There are many strategies for the model fitting for deterministic computer experiments, such as Gaussian process models (also known as kriging), polynomial models (also known as response surface models), radial basis function models, multivariate adaptive regression splines (MARS) and neural networks. The choice of a model fitting technique is typically based on the current knowledge on how a specific system operates (Chen et al., 2002).

Polynomial models, or response surface models, were first developed by Box and Wilson (1951). In their paper, they used first and second order polynomials to approximate the underlying models. The main idea of this approach is to use a sequence 
of experimental sub-regions to obtain an optimal response. Polynomial models are ideal ways for uncertainty analysis; they are easy to estimate and apply even when little is known about the problem. However, for the problems which are highly nonlinear, multimodal, or multidimensional, polynomial models may not be suitable to apply. Also, polynomial models normally offer limited information about where to add samples to improve the model (Forrester et al., 2008). Gaussian process (GP) regression model, so-called 'Kriging', has its origins in geostatistics (Matheron, 1963). Sacks et al. (1989) introduced the application of Kriging to computer experiments, and this approach is now widely used for obtaining the metamodels (Santner et al., 2013). The basic idea of kriging is using an interpolating predictor that the predicted surface can interpolate the observed responses. This interpolation seems ideal for the deterministic computer experiments; however, drawbacks still exist in using kriging: One of the problems is that the model fitting is slower than the polynomial models. Also, assumptions of the correlation function are difficult to verify (Chen et al., 2002); once the correlation parameters are misspecified, the kriging model will not be robust. Joseph et al. (2008) proposed a modified form of Kriging, called blind Kriging. The idea of blind Kriging is to use an unknown mean model instead of the constant mean of the ordinary Kriging. The application examples in their paper showed that a blind Kriging model is easier to interpret and more robust.

Friedman (1991) introduced multivariate adaptive regression splines (MARS), using non-parametric regression technique. Dyn et al. (1986) described radial basis functions; under certain assumptions, they are able to build global approximation surfaces to interpolate smooth data. Lippmann (1987) introduced the approach of neural network. Simpson et al. (2001) considered the use of neural network in computer experiments and they commented that 'Neural networks are best for approximating deterministic functions in regression-type applications'. Neural networks have been applied 
with a large quantity of parameters, however, 'the requisite gathering of training data and calculation of model parameters can be extremely computationally expensive' (Simpson et al., 2001). There many other alternative model fitting techniques. Wang and Shan (2007) presented a list of commonly used surrogate model choices; and they discussed their application to engineering design and optimizations.

\subsubsection{Search the Optima Based on the Surrogate Model}

After the surrogate model is established, optimization tools are applied to search the optimal solution(s) of the system. Existing optimization techniques are commonly classified into, for example, global searchers as compared to local optimizers (Tekin and Sabuncuoglu, 2004); or gradient-free optimization approaches as compared to gradient-based approaches (Rubinstein, 1997). For unimodal smooth functions, local optimizers are very efficient. However, for multimodal functions, when there are many valleys or multiple local optima, local optimizers often provide less than satisfactory results (Forrester et al., 2008).

Under the category of local optimizers, they are divided into two groups: gradientbased local optimizers, and gradient-free local optimizers. Those gradient-based local optimizers, which use slope information to explore the best path towards the local optimum, include the Newton method, quasiNewton methods (Wedderburn, 1974) and conjugate gradient optimizers (Hestenes and Stiefel, 1952). For those local optimizers without knowing the gradient information are also called direct search. These include the Simplex method, the complex method (Box, 1965) and the pattern search (Hooke and Jeeves, 1961).

Compared to local optimizers, global searchers are aimed to find the global optima, including genetic algorithms (Golberg, 1989), simulated annealing (Kirkpatrick et al., 1983), particle swarm optimization (PSO) (Eberhart and Kennedy, 1995), etc. 
Current trend has been towards gradient-free global searchers, for the gradient information is not always available or accurate due to noises. In recent years, hybrid algorithms with a combination of global and local have been proposed: Campana et al. (2009) proposed a two phase global-local approach based on the PSO.

\subsection{Thesis Overview}

The SeaWEED wave energy converter, which was invented by Grey Island Energy Incorporation in Newfoundland, Canada is currently under development in its second generation. It is an oscillating body type wave energy converter with multiple bodies. The converter has three hinged parts consisting of four modules that are connected by rigid truss structures. The electric power is generated by the vertical hinge motions at two hinged joints where the power take-off (PTO) system is located. After the model tests for the first generation of SeaWEED, improvements have been made and it led to the design of the second generation, which was numerically evaluated and optimized in this thesis.

For the optimization objective of SeaWEED, it is an early stage of the design that a detailed cost function for the device is not feasible. Thus, a single objective was set as to maximize the power absorption performance of the device. The optimization of the device focused both on the PTO damping and the geometrical parameters in terms of the draft and the length of the interconnecting truss structures.

During the optimization process, a critical problem is that the optimal PTO parameter is related to the added-mass and hydrodynamic damping, as derived by Evans (1976), which is further related to the underwater geometry of the device. In other words, the variable of PTO damping is not independent of the geometrical parameters. Therefore, they are not able to be set as optimization variables at the same 
time.

For our problem, two-step iterative optimization was implemented:

- At the very beginning, a sampling plan of truss length L and draft T combinations was determined. Each combination of $\mathrm{L}$ and $\mathrm{T}$ defines a geometry of the model. In the first step of optimization, the variable is the PTO damping and it is optimized for each geometry.

- In the second step, the variables are truss length $\mathrm{L}$ and draft $\mathrm{T}$. The corresponding optimal damping value is iterated in each geometry. Therefore, for a certain wave condition, the truss length and draft are optimized along with the corresponding PTO damping value.

Both full factorial design and uniform design were used in this thesis. The capacity of the computer allows full factorial experiments with three parameters and five levels for each parameter. Full factorial design gives more information on how the variables effect the responses and the interaction between each other. Uniform design was used based on the knowledge to the problem and the model which is more efficient. For each design, the polynomial regression analysis was utilized to fit the response surface in the software of Design-Expert. Additional simulation experiments were conducted to verify the surrogate model. The optimal solutions were searched by using a global optimization method which is known as Derringer's desirability function developed by Derringer and Suich and described in the book of Response Surface Methodology by Myers et al. (2009).

An overview of each chapter is presented as following:

Chapter 1 presents a review of the development of wave energy converters and simulation-based optimization design methodologies.

Chapter 2 introduces the SeaWEED WEC in its first and second generations. The 
special designed PTO mechanism of SeaWEED is illustrated.

Chapter 3 derives the mathematical formulations of the boundary value problem of floating hinged arrays and presents the mathematical description of the power take-off system. The power absorption formulations of the hinged type WEC in regular waves and in irregular sea states are derived.

Chapter 4 describes the 1:16 scale model test and the frequency domain simulation. The experimental results are compared with the numerical solutions to validate the numerical approach. Also, a convergence study is presented to verify the the numerical approach and to determine an appropriate panel number for the numerical modeling of the second generation of SeaWEED.

Chapter 5 discusses the optimization of the second generation SeaWEED in terms of the PTO optimization and the geometrical optimization. The full factorial design and uniform design were applied and the surrogate model was fitted by polynomial regression analysis. The optimal PTO damping and optimal truss length and draft were determined in regular waves in order to maximize the absorbed power. The optimal parameters and maximized power absorption in an intended location are computed.

Chapter 6 concludes the work that has been done and discusses the limitations of current work. 


\section{Chapter 2}

\section{SeaWEED Wave Energy Converter}

\subsection{An Introduction of SeaWEED}

SeaWEED (Sea Wave Energy Extraction Device) is a multi-body attenuating WEC, extracting power by allowing relative pitch movements between adjacent bodies. The first generation of SeaWEED is shown in Figure2.1.

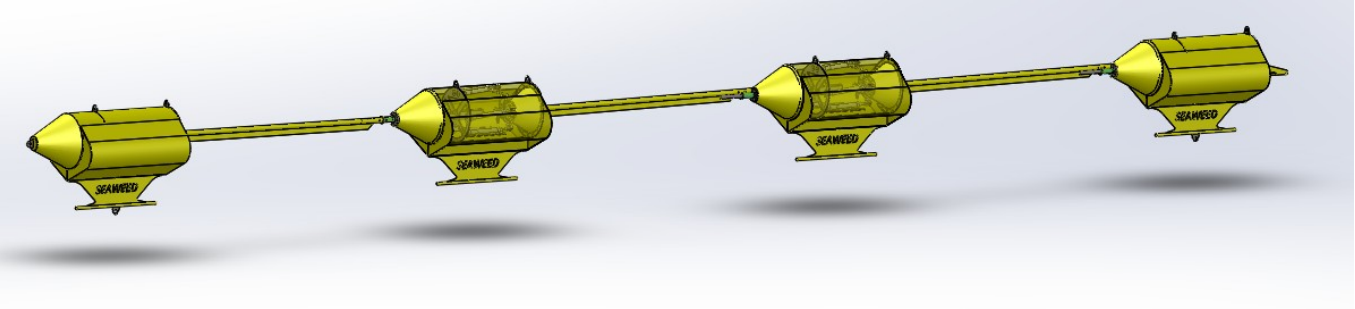

Figure 2.1: The first generation of SeaWEED system.

A complete SeaWEED system is a four module array consisting of a non-energy producing nose module in the front, followed by two energy producing modules, with another non-energy producing module at the rear. Two hydraulic PTO systems are positioned at the stern of two producing modules. The four modules are at the same length and are connected by the tie-rods. Figure 2.2 illustrates the SeaWEED PTO 
and its placement in the SeaWEED system.

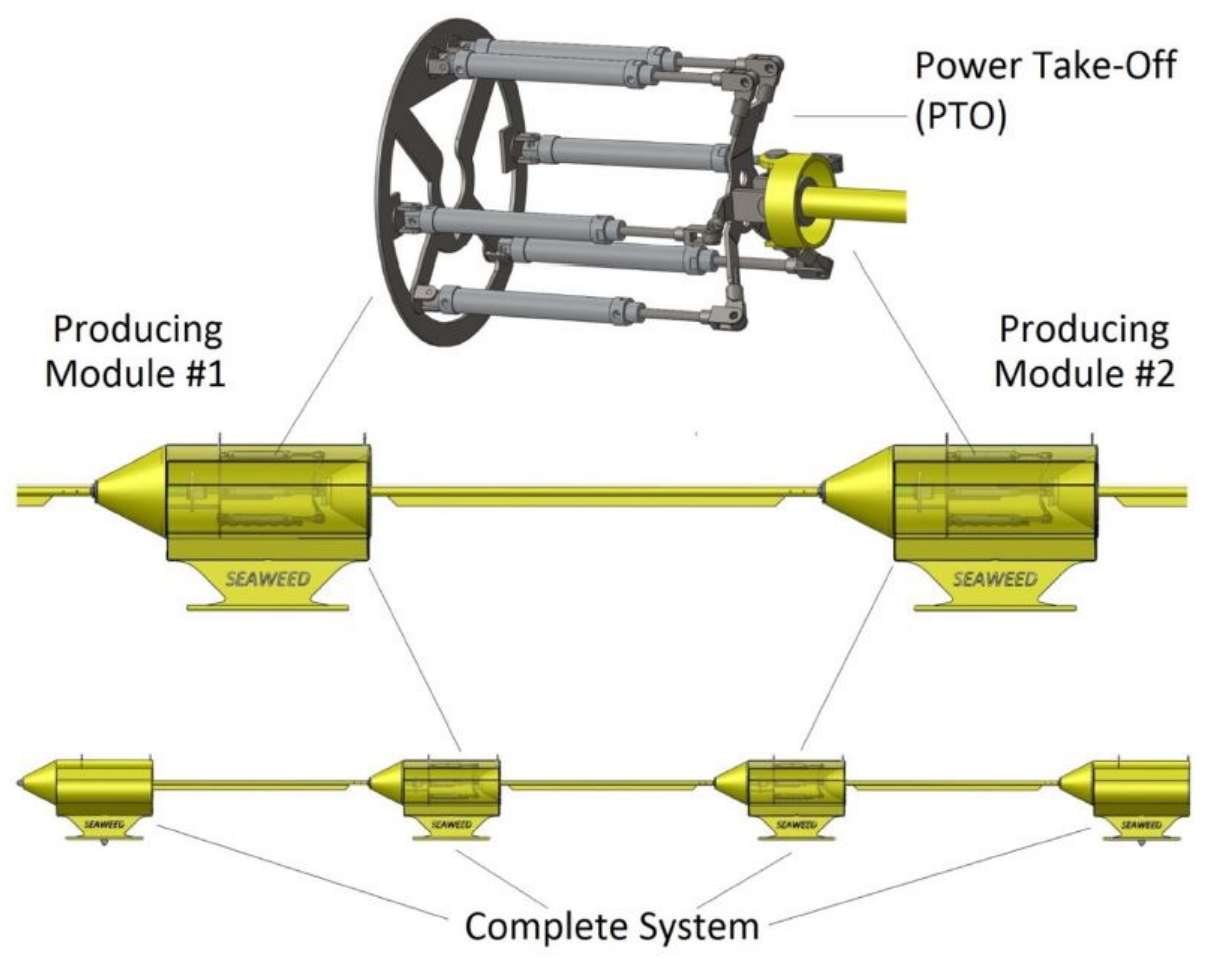

Figure 2.2: Illustration of the PTO and the complete first generation SeaWEED system.

After the model tests for the first generation of SeaWEED, improvements have been made and it led to the design of the second generation, which was numerically evaluated and optimized in this thesis. Note that the PTO mechanism remains the same as that in the first generation. A fully assembled second generation of SeaWEED device is presented in Figure 2.3. The geometry of the hull modules was modified by using flat panel to reduce fabrication cost. Also the connecting parts were enhanced by using truss structure. As shown in Figure 2.4, the second generation of SeaWEED device consists of four modules that are connected by rigid truss structures. SeaWEED is designed to operate in a water depth of approximately 50 meters depending on the connecting member length and the sea spectrum of the area of interest. The SeaWEED system will utilize onboard electrical generator and standard subsea 


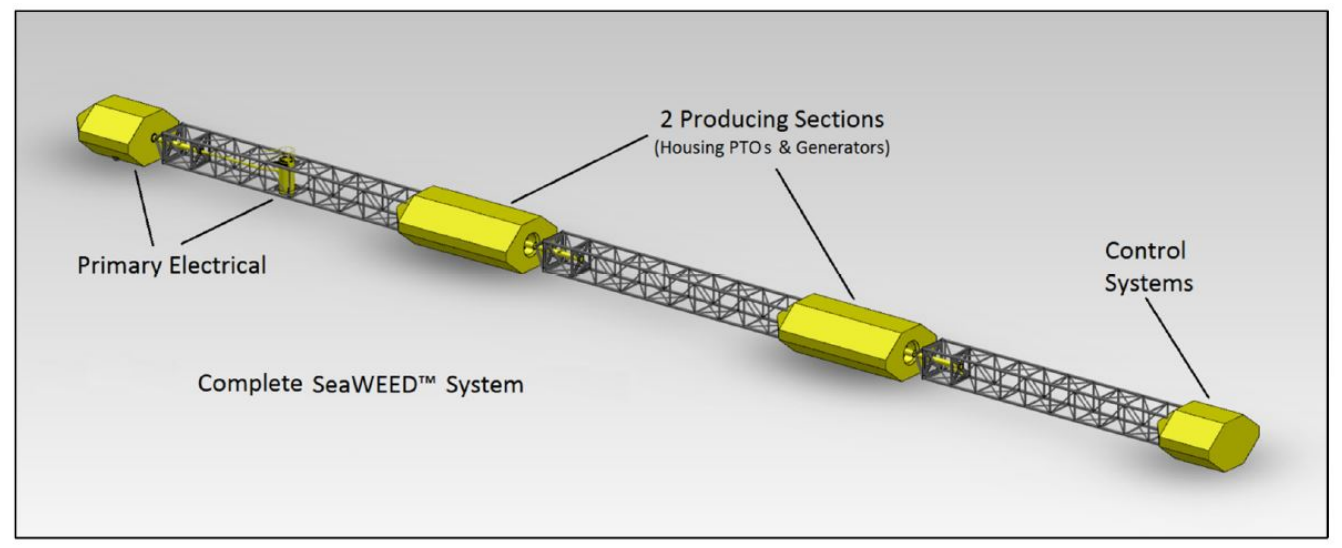

Figure 2.3: Fully assembled second generation of SeaWEED system.

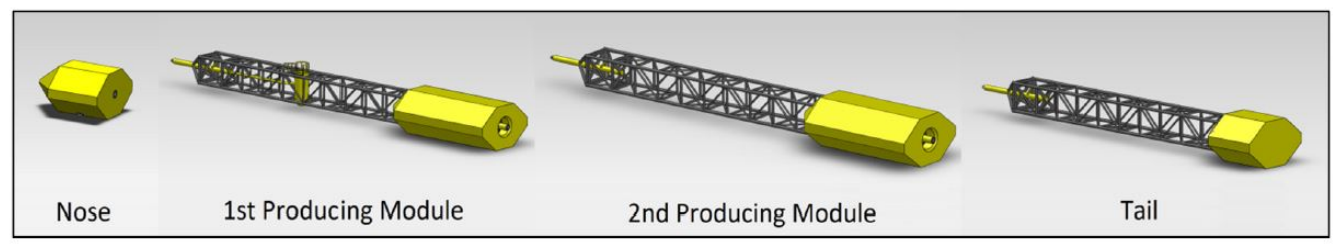

Figure 2.4: Four modular sections of SeaWEED.

electrical cables to generate and transmit the energy to shore. The cost of this subsea electrical cable can be significant compared to the cost of a single SeaWEED system. However, only one cable to shore is required for a SeaWEED farm, which becomes cost effective as additional SeaWEED devices are installed. An offshore farm of SeaWEED WECs can be utilized to provide electricity to coastal communities; it can also be utilized by Oil and Gas Companies to power autonomous offshore oil rigs or subsea infrastructure. In the real operating environment, SeaWEED will experience several types of motion which are heave and pitch, roll, and yaw. The electrical power is only converted from the vertical pitch motion, which is called hinge motion in this thesis. 


\subsection{Power Take-Off Mechanism of SeaWEED}

The hydraulic PTO components of SeaWEED are completely internalized in the producing modules, utilizing a water tight multi-axis joint in the stern of each module a single bellows surrounding the joint as added protection. This design will not only protect all PTO components from the harsh marine environment but also prevent the environment contamination by the leak of the system's hydraulics. Figure 2.5 illustrates the power take-off system of SeaWEED.

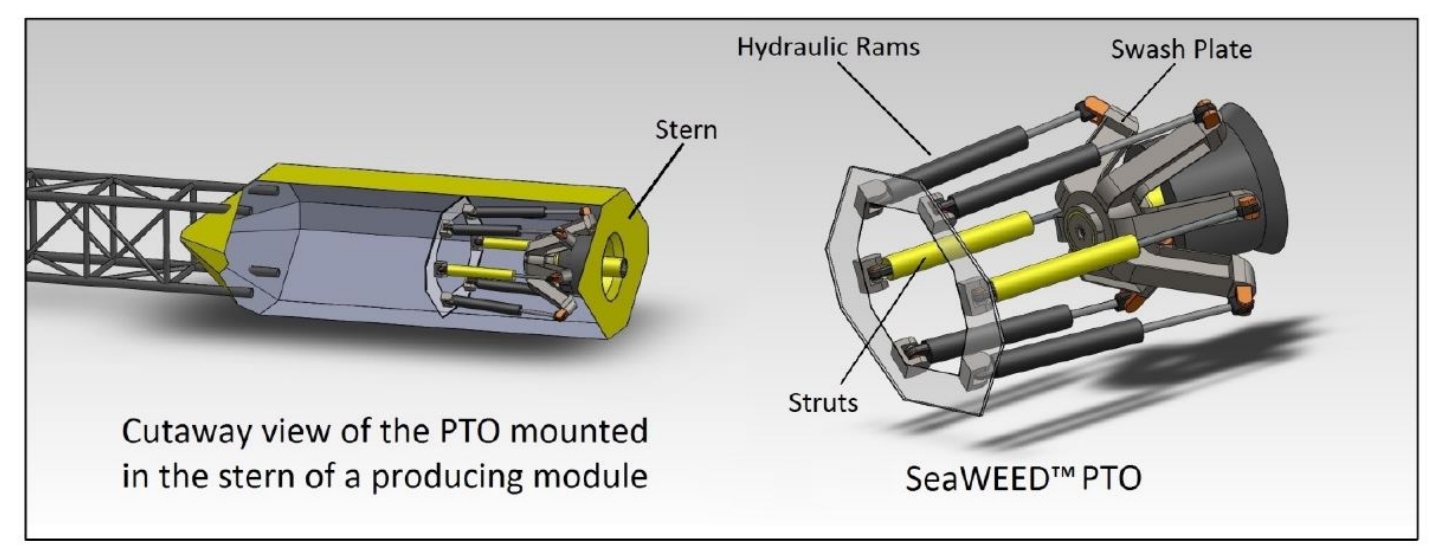

Figure 2.5: SeaWEED power take-off system.

The SeaWEED PTO system is driven by movements between interconnected modules. A multi-axis joint is located in the stern of a producing module. The relative motion between interconnected modules drives a swash plate located internally to tilt in various planes and thus compress or extend the hydraulic rams. The hydraulic rams work in conjunction with struts to harness energy and contribute to system movements. This energy is captured by the upper and lower pairs of hydraulic rams (in black) as illustrated in Figure 2.6.

The design of attenuator devices normally allows flexibility in all directions in order to relieve stresses that may lead to joint fatigue. For floating attenuators in waves, the vertical motion such as heave and pitch is restorable since gravity provides the 


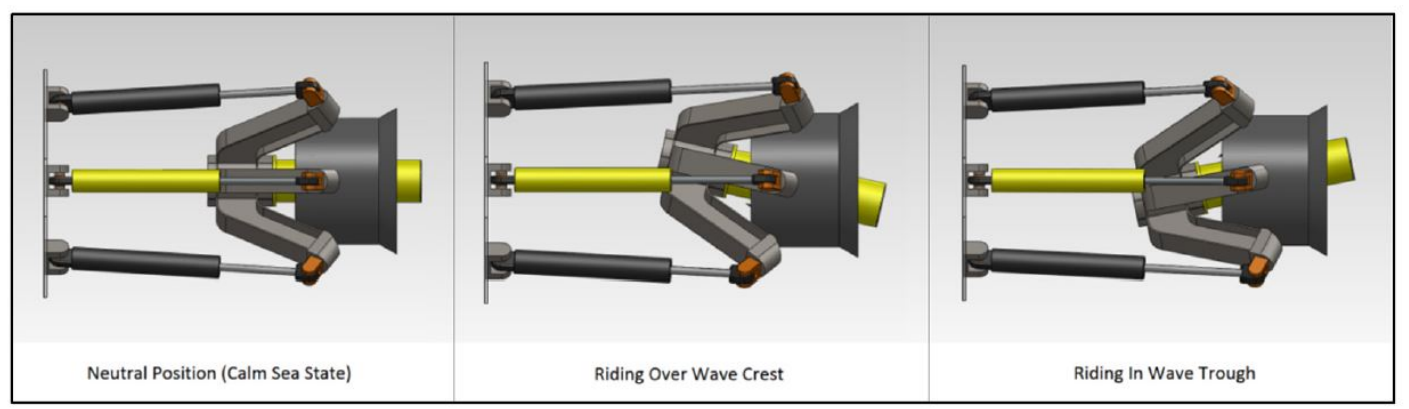

Figure 2.6: Profile views of the SeaWEED hydraulic power take-off system.

restoring force. However, for horizontal motion as yaw, there is no significant restoring force to bring the device back to its neutral position. Therefore, some form of artificial force is needed to realign the system horizontally once a wave has passed. Pelamis Yemm et al. (2012) accomplished the restoring of both pitch and yaw by inclining the system to the water surface with a roll bias angle, as shown in Figure 2.7. For the first generation of Pelamis, P1, the joint did not allow for a gentle restoration of the system to a neutrally aligned position, causing fatigue in the joints and resulted in failure over time. As a result of the P1's failure, the PTO joint section was redesigned. The second generation of Pelamis, P2, utilized a single multi-axis joint system, which was repositioned closer to the centre of the module, allowing for better system flexibility and altered the position of the four hydraulic power capture rams to compensate for system realignment, as shown in Figure 2.8. The redesign of P2 successfully reduced the joint fatigue compared to P1, however, it has also significantly added to the P2's cost and reduced the amount of energy capture.

For such multi-body attenuating WECs, the power capture in yaw direction may not be economical considering the cost and risk. Therefore, SeaWEED is to strictly capture the purest form of ocean energy in the vertical motion, allowing free roll motion and fluidic damped yaw motion. 


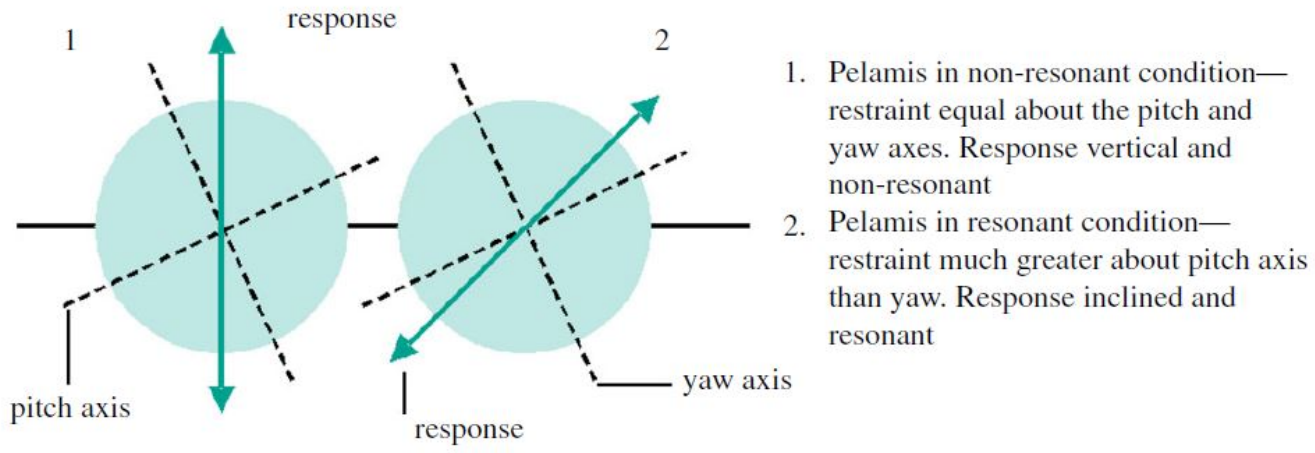

Figure 2.7: Schematic of Pelamis selectable-tunable joint response (Yemm et al., 2012).

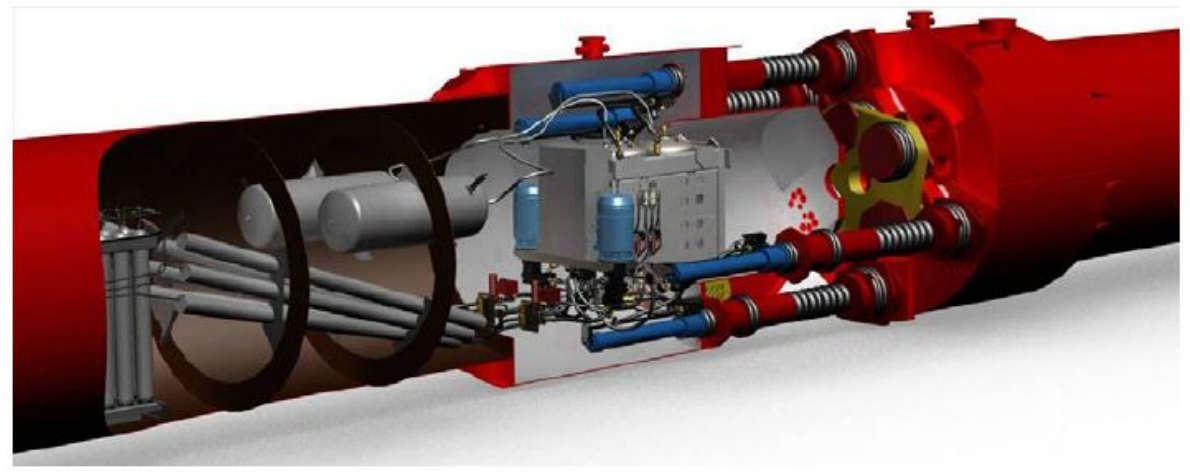

Figure 2.8: Pelamis P2 hydraulic PTO joint (Yemm et al., 2012). 


\subsection{Competitive Advantages}

The design of SeaWEED is aimed to be economical and easy to manufacture, install and maintain. For different wave climates, the best structure length might be varied. To manufacture the device for different locations, the overall length is able to be tuned by the connecting member length. SeaWEED is constructed in modules, and the efficient manufacturing and installation can be performed in any small or medium sized dockyard. SeaWEED also has a storm mode system that allows the device to semi-submerge and ride out adverse storm conditions, yet ensuring the safety and an optimal energy output. 


\section{Chapter 3}

\section{Theoretical Background and Mathematical Formulation}

\subsection{Motion of Hinged Bodies in Waves}

\subsubsection{Two General Approaches for Multi-Body System}

For multi-body system with constrained joints in waves, there are two types of approaches that can be adopted based on the potential flow theory. The first type of approach is regarded as a 'two-step' approach (Sun et al., 2011). For the first step, the diffraction problem without constraints is solved while the bodies are allowed to oscillate freely in all of their rigid body degrees of freedom (DOF). The total number of DOF is six times the number of bodies. After the wave excitation forces and the hydrodynamic coefficients are solved, in the second step, the method of connection between the modules is considered and the motion equations with constraints are solved. A detailed derivation and application of this 'two-step' approach was presented by Langley (1984). This approach is highly flexible when solving a multi-body system with complex constraints. 
The second approach to analyze the multi-body system is to directly solve the cou-

pled diffraction and radiation problems with consideration of the connection and constraints, as discussed by Newman (1994) and Lee and Newman (2000). This method is more efficient as it considers fewer DOFs than the first one so that fewer radiation problems need to be solved. For the multi-body system of SeaWEED WEC with hinged joints, the second approach is applied.

\subsubsection{Newman's Approach for Hinged Arrays}

Motion of a hinged array with identical rigid modules and uniform mass distribution was discussed by Newman (1997). Here, we will focus on general hinged arrays with rigid modules in different lengths and non-uniform mass distribution.

Assume there are $N$ rigid bodies connected together by hinged joints ideally without friction. Each body is one module in the hinged array. The conventional Cartesian coordinate system is applied to the hinged array, with $x=0$ at the midpoint of the array, $y=0$ at the vertical centerplane, z-axis upwards, and $z=0$ at the undisturbed water surface.

As shown in Figure 3.1, $N$ bodies are connected by $N-1$ hinge joints. Two ends and the hinge joints from stern to bow are defined as $x_{0}, x_{1}, \ldots x_{n}, \ldots, x_{N}$. The hinge joints are located at $x_{1}, x_{2}, \ldots, x_{n}, \ldots, x_{N-1}$. The length of each module between two adjacent joints can be different.

A hinge mode is defined as the elevation of the corresponding hinge joint while other joints remain at $z=0$. For $N$ hinged modules, there are $N-1$ hinge modes. Figure 3.1 shows the definition of general hinge modes by giving an example of an array with four hinged modules.

It is seen from Figure 3.2 that each hinge mode is only to do with two adjacent bodies. In head-sea states, conservative vertical motions in terms of heave and pitch are taken 


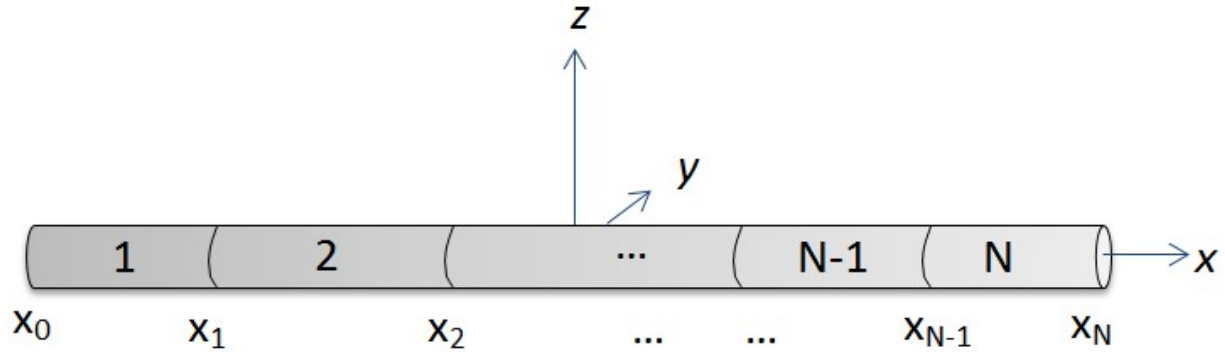

Figure 3.1: An array of hinged modules.

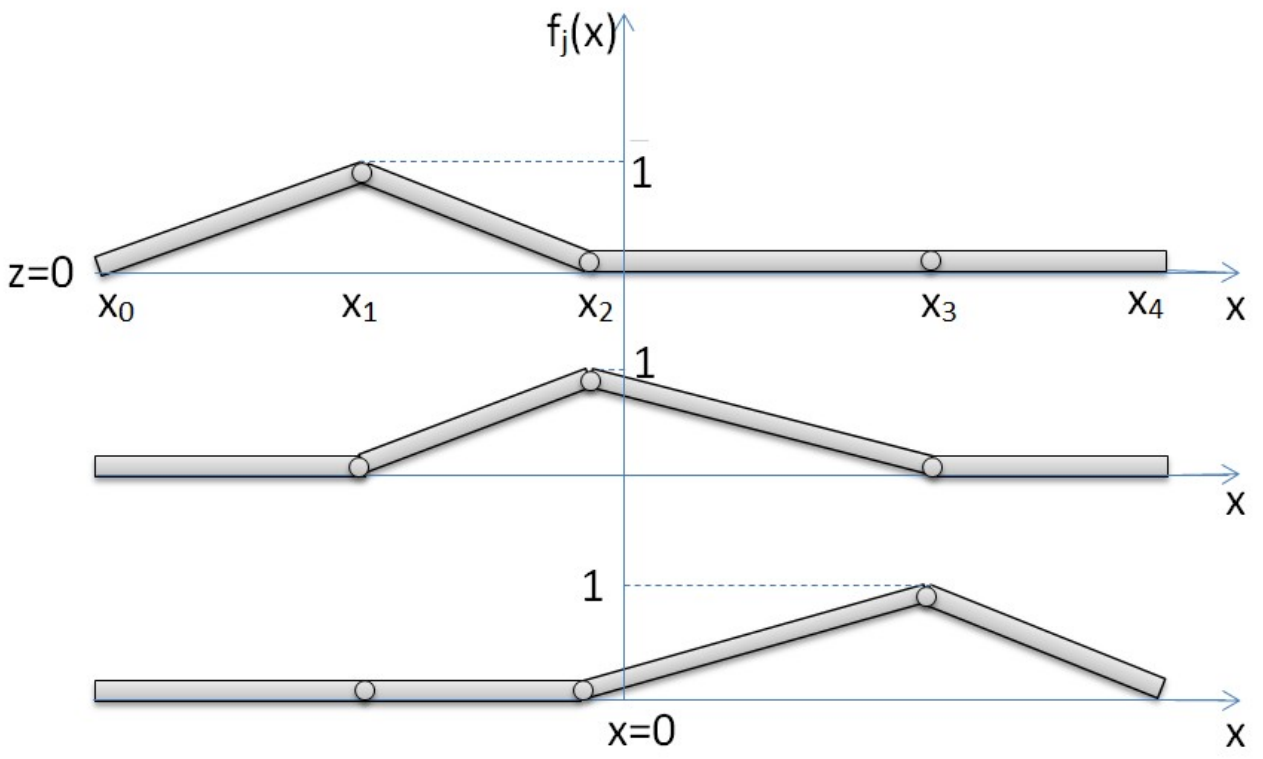

Figure 3.2: Illustration of general hinge modes. 
into consideration as they might be coupling with the hinge modes. The heave and pitch modes are illustrated in Figure 3.3.
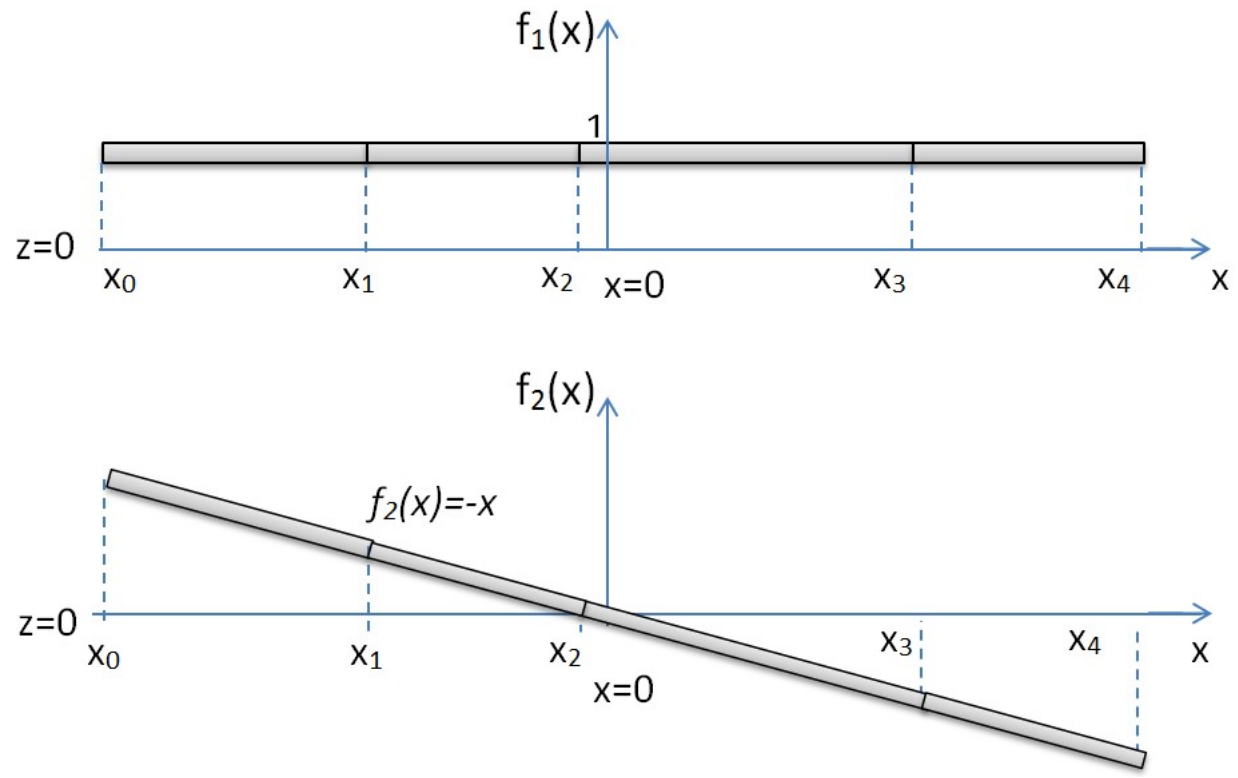

Figure 3.3: Illustration of heave and pitch modes.

For a hinged array with $N$ modules, there are $2+(N-1)$ modes of interest in headsea states. The first two modes are defined as heave and pitch while the next $N-1$ modes are the hinge modes. Mathematically, each of the heave, pitch and all the hinge modes can be represented by the shape function: $f_{j}(x), j=1, \ldots, N+1$. $f_{j}(x)$ describes the shape of mode with unit amplitude. The formulated expression of the vertical modes is as following:

Heave, when $j=1$, is expressed as:

$$
f_{1}(x)=1
$$

Pitch, when $j=2$, is expressed as:

$$
f_{2}(x)=-x
$$


Hinge mode at $x_{n}, n=1,2,3, \ldots, N-1$ is expressed by the tent function, as shown in Figure 3.4. When $j=n+2, n=1,2,3, \ldots, N-1$ hinge modes are expressed as

$$
\begin{gathered}
f_{n+2}(x)=\frac{1}{x_{n}-x_{n-1}}\left(x-x_{n-1}\right), x_{n-1} \leq x<x_{n} \\
f_{n+2}(x)=\frac{1}{x_{n}-x_{n+1}}\left(x-x_{n+1}\right), x_{n} \leq x<x_{n+1} \\
f_{n+2}(x)=0, x<x_{n-1} \text { or } x \geq x_{n+1}
\end{gathered}
$$

where $n+2$ is the index of the hinge mode within $[3, N+1]$.

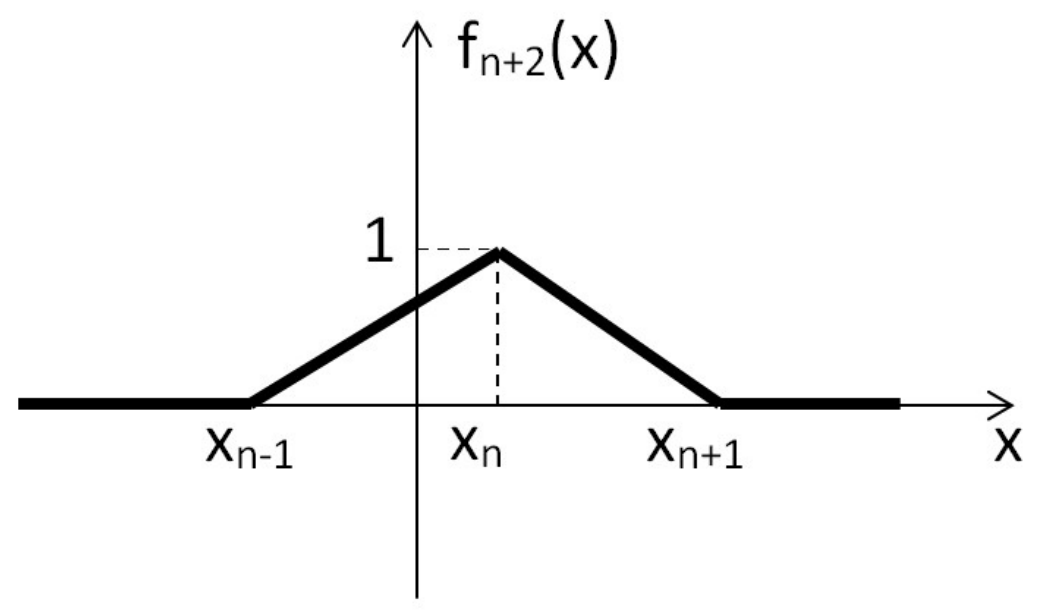

Figure 3.4: General tent function.

\subsubsection{Mass Matrix of a Hinged Array}

Newman (1997) defined the mass matrix $M_{i j}$ to represent the internal mass distribution of the hinged array. He derived the shape functions of hinge modes which are symmetric or anti-symmetric about the mid-ship section, and formulated the nondimensional mass matrix for the hinged array. Here, the derivation of $M_{i j}$ focused on general asymmetric hinge modes. Each general mode $f_{j}(x)$ is defined by the tent 
function, as shown in Figure 3.4, and the dimensional mass matrix $M_{i j}$ is calculated by

$$
M_{i j}=\int_{L} m(x) f_{i}(x) f_{j}(x) d x
$$

where $L$ is the total length of the array, $i$ and $j$ are indices of each mode including heave, pitch and hinge modes. $i=1,2, \ldots, N+1, j=1,2, \ldots N+1, N$ is the number of the bodies in the array. $M_{i j}$ is an orthogonal matrix.

The terms of the mass matrix $M_{i j}$ are formulated as following,

$$
\begin{gathered}
M_{11}=\int_{x_{0}}^{x_{N+1}} m(x) d x \\
M_{22}=\int_{x_{0}}^{x_{N+1}} m(x) x^{2} d x \\
M_{n+2, n+2}=\int_{x_{n-1}}^{x_{n}} m(x)\left(\frac{x-x_{n-1}}{x_{n}-x_{n-1}}\right)^{2}+\int_{x_{n}}^{x_{n+1}} m(x)\left(\frac{x_{n+1}-x}{x_{n+1}-x_{n}}\right)^{2} d x \\
M_{n+2,(n+1)+2}=\int_{x_{n}}^{x_{n+1}} m(x)\left(\frac{x_{n+1}-x}{x_{n+1}-x_{n}}\right)\left(\frac{x-x_{n}}{x_{n+1}-x_{n}}\right) d x=M_{(n+1)+2, n+2} \\
M_{2, n+2}=\int_{x_{n-1}}^{x_{n}} m(x)(-x) \frac{x-x_{n-1}}{x_{n}-x_{n-1}} d x+\int_{x_{n}}^{x_{n+1}} m(x)(-x) \frac{x_{n+1}-x}{x_{n+1}-x_{n}} d x=M_{n+2,2} \\
M_{1, n+2}=\int_{x_{n-1}}^{x_{n}} m(x) \frac{x-x_{x_{0}}^{x_{N+1}}}{x_{n}-x_{n-1}} d x+\int_{x_{n}}^{x_{n+1}} m(x) \frac{x_{n+1}-x}{x_{n+1}-x_{n}} d x=M_{n+2,1}
\end{gathered}
$$


where $n=1,2, \ldots, \mathrm{N}-1$.

$M_{i j}$ is symmetric. $M_{11}$ and $M_{22}$ are the heave and pitch terms on the diagonal of the matrix. $M_{n+2, n+2}$ is the term of each hinge mode on the diagonal. $M_{n+2,(n+1)+2}$ is the off-diagonal coupled term between two adjacent hinge modes. $M_{12}, M_{1, n+2}, M_{2, n+2}$ and their symmetrical terms are the off-diagonal coupled terms between conventional modes and hinge modes. The rest of the terms are zero. Knowing the mass distribution $m(x)$ of the array, the mass matrix can be obtained by doing segmental integral of equation 3.6.

\subsubsection{Boundary Value Problem of Hinged Arrays}

For floating WECs with hinged bodies, it is the relative motion between each body that drives the power take-off (PTO) system to convert mechanical power into electricity. To evaluate the relative hinge motions, the boundary value problem for the hinged array needs to be solved.

The following theory is based on the assumptions that:

- The boundary conditions are linearized, i.e., linear body surface condition, linear free surface condition and linear bottom condition.

- The hydrodynamic force components are linearized, i.e., linear radiation force, linear diffraction force, linear Froude-Krylov force and linear restoring force.

- The viscosity of the fluid is assumed to be neglected so that the potential flow theory is adopted.

In potential theory, the fluid velocity is described by the gradient of the velocity potential $\Phi$, which is governed by Laplace's equation in the fluid domain.

$$
\nabla^{2} \Phi=0
$$


The harmonic time dependence allows the definition of a complex velocity potential $\varphi$, related to $\Phi$ by

$$
\Phi=\operatorname{Re}\left(\varphi e^{i \omega t}\right)
$$

where Re denotes the real part, $\omega$ is the frequency of the incident wave and $t$ is the time. The boundary value problem will be expressed in terms of the complex velocity potential $\varphi$ while the product of all complex quantities are with the factor $e^{i \omega t}$. The linearized free surface boundary condition is

$$
\frac{\partial \varphi}{\partial z}-K \varphi=0 \quad \text { on } z=0
$$

where $K=\omega^{2} / g$ is the infinite-depth wave number and $g$ is the acceleration of gravity. The bottom condition is

$$
\frac{\partial \varphi}{\partial z}=0 \quad \text { on } z=-h
$$

where $h$ is the water depth.

On the lateral boundaries, radiation conditions are statisfied.

The linearization of the problem permits the decomposition of the velocity potential $\varphi$ into three components,

$$
\varphi=\varphi_{I}+\varphi_{R}+\varphi_{D}
$$

where $\varphi_{I}$ is the incident wave potential, $\varphi_{R}$ is the radiation potential and $\varphi_{D}$ is the diffraction potential.

For incident wave potential, it is calculated by

$$
\varphi_{I}=\frac{i g A}{\omega} \frac{\cosh [k(z+h)]}{\cosh k h} e^{-i k(x \cos \beta+y \sin \beta)}
$$

where $\omega$ is the wave frequency, $g$ is the acceleration of gravity, $A$ is wave amplitude, 
$\beta$ is the angle of the incident wave related to the $\mathrm{x}$-axis of the Cartesian coordinate system, $k$ is the wavenumber which is calculated from

$$
\frac{\omega^{2}}{g}=k \tanh k h
$$

where $h$ is the water depth. For deep water cases, the incident wave potential can be written as

$$
\varphi_{I}=\frac{i g A}{\omega} e^{k z-i k(x \cos \beta+y \sin \beta)}
$$

where the wavenumber $k=K$.

The radiation potential is defined as

$$
\varphi_{R}=i \omega \sum_{j=1}^{J} \xi_{j} \varphi_{j}
$$

where $\xi_{j}$ represents the complex motion amplitude of mode $j$, and $\varphi_{j}$ is the corresponding unit-amplitude radiation potential of mode $j, j=1,2,3, \ldots, J$. J represents the number of modes including six conservative modes and extra hinge modes. In WAMIT, with consideration of six conventional modes: surge, sway, heave, roll, pitch, yaw, the hinge modes are defined to be from mode 7 to mode $J$.

The boundary conditions of the radiation problem are as following: In the fluid domain, the Laplace equation should be satisfied

$$
\nabla^{2} \varphi_{j}=0
$$

On the free surface where $z=0$, the boundary condition satisfies

$$
\frac{\partial \varphi_{j}}{\partial z}-K \varphi_{j}=0
$$


On the body surface, the boundary condition satisfies

$$
\frac{\partial \varphi_{j}}{\partial n}=n_{j}
$$

where $n_{j}$ is the normal component of the displacement on the body surface of mode $j .\left(n_{1}, n_{2}, n_{3}\right)=\mathbf{n},\left(n_{4}, n_{5}, n_{6}\right)=\mathbf{x} \times \mathbf{n}$, and for vertical hinge mode $n_{j}=f_{j} n_{z}$. For diffraction problem, the corresponding boundary conditions are as following. In the fluid domain

$$
\nabla^{2} \varphi_{D}=0
$$

On the free surface where $z=0$

$$
\frac{\partial \varphi_{D}}{\partial z}-K \varphi_{D}=0
$$

On the body surface

$$
\frac{\partial \varphi_{D}}{\partial n}=-\frac{\partial \varphi_{I}}{\partial n}
$$

The corresponding forces to each mode is calculated by

$$
F_{i}=\iint_{S_{b}}^{\prime} p n_{i} d S
$$

where $p$ is the fluid pressure, $S_{b}$ is the body boundary surface. According to Bernoulli equation, the pressure is calculated from

$$
p=-\frac{\rho v^{2}}{2}-\rho g z-\rho \frac{\partial \varphi}{\partial t}+p_{a}
$$

where the $p_{a}$ term is neglected since the integration over the wetted surface is zero. The first term evaluates a second-order pressure. The second term evaluates the 
hydrostatic pressure and the third term evaluates the hydrodynamic pressure in waves. After linearization, the first-order pressure can be represented by

$$
p=-\rho \frac{\partial \varphi}{\partial t}-\rho g z=-\rho i \omega \varphi-\rho g z
$$

where $z$ is the vertical axis of Cartesian coordinates. Substitute equation 3.31 to equation 3.29 ,

$$
F_{i}=-\rho \iint_{S_{b}}^{\prime}(i \omega \varphi+g z) n_{i} d S
$$

The added-mass and damping matrices are defined by the unit-amplitude radiation potential related to the first term of equation 3.32 ,

$$
\omega^{2} a_{i j}-i \omega b_{i j}=-i \omega \rho \iint_{S} \varphi_{j} n_{i} d S
$$

where $a_{i j}$ is the added-mass matrix and $b_{i j}$ is the hydrodynamic damping matrix. Both $i$ and $j$ are in the values from $1,2, \ldots 6$ and $7,8 \ldots J$, where $J$ is the number of modes including extra hinge modes.

The wave-exciting force is calculated from

$$
X_{i}=-i \omega \rho \iint_{S_{b}}\left(\varphi_{I}+\varphi_{D}\right) n_{i} d S
$$

For hydrostatic force, it can be calculated from restoring matrix $c_{i j}$ which is defined by the second term of equation 3.32 ,

$$
c_{i j}=\rho g \iint_{S_{b}} z n_{j} d S
$$

For a hinge mode, we defined its shape function by $f_{i}(x)$ which represents the vertical displacement along $\mathrm{x}$-axis of mode $i$. Thus the vertical displacement $z$ in equation 
3.35 can be replaced by $f_{i} . n_{j}$ is defined as the normal component of displacement on the surface. For hinge mode, only vertical displacement exits, thus

$$
n_{j}=f_{j} n_{z}
$$

Therefore, for hinge modes, the hydrostatic restoring matrix is represented by integrating the shape function along the length

$$
c_{i j}=\rho g \iint_{S_{b}} f_{i}(x) f_{j}(x) n_{z} d S
$$

Therefore, a linear system of motion equations is given in the following form:

$$
\sum_{j=1}^{J} \xi_{j}\left[-\omega^{2}\left(M_{i j}+a_{i j}\right)+i \omega b_{i j}+c_{i j}\right]=X_{i}
$$

where both $i$ and $j$ are in the values of $1,2, \ldots, 6,7,8 \ldots J$.

Solving the linear equation system of equation 3.38 will get the motion amplitude $\xi_{j}$ for each mode (Newman, 1994).

In the thesis, the motion of the hinged device was solved by using WAMIT, a commercial software based on potential flow theory. WAMIT defines six conservative modes which are surge, sway, heave, roll, pitch and yaw. The extra hinge modes are defined from 7 to $N+5$, assuming there are $N$ hinged parts. The mass matrix of the hinged array needs to be input by users. In the head-sea condition, mode 3 (heave), mode 5 (pitch) and the hinge modes from 7 to $N+5$ are of interest. 


\subsection{Numerical Modeling of Power Take-Off system}

\subsubsection{Power Absorption in Regular Waves}

The mechanism of power extraction can be modelled by a linear spring and damper system (Evans, 1976). A linear external damping coefficient is applied to enable power absorption and an external spring force is to tune the device to the incoming wave conditions. The system is represented by

$$
m \ddot{\xi}_{i}+d_{i} \dot{\xi}_{i}+k_{i} \xi_{i}=F_{i}
$$

where $d_{i}$ is the PTO damping for the $i^{t h}$ mode, $k_{i}$ is the PTO stiffness for the $i^{t h}$ mode. Note that in the design of SeaWEED PTO system, there is no PTO stiffness in the hydraulic rams thus $k_{i}=0 . m$ is the mass parameter of the structure; $\xi_{i}$ is the motion amplitude of mode $i$; and $F_{i}$ is the hydrodynamic force of mode $i$ defined by equation 3.29. The hydrodynamic forces corresponding to each mode of motion consist of radiation component(added mass and hydrodynamic damping), wave-exciting component and hydrostatic component.

$$
F_{i}=F_{r a d, i}+X_{i}+F_{r e s, i}
$$

where $F_{r a d, i}$ and $F_{r e s, i}$ are the $i^{\text {th }}$ mode of the radiation force and restoring force; $X_{i}$ is the corresponding wave exciting force including the diffraction force and the incident wave force (Froude-Krylov force).

The PTO force is contributed by the term with PTO damping,

$$
F_{P}=d_{i} \dot{\xi}_{i}
$$


where $i$ is the mode that contributes to the power absorption.

The time-averaged absorbed power per unit length by the device is calculated by

$$
\bar{P}=\frac{1}{T} \int_{T} F_{P} \cdot \dot{\xi}_{i} d t=\frac{1}{T} \int_{T} d_{i} \dot{\xi}_{i} \dot{\xi}_{i} d t=\frac{1}{2} \omega^{2} d_{i}\left|\xi_{i}\right|^{2}
$$

The total available wave power of incident wave per meter of crest in regular waves is (Thorpe, 1999)

$$
P_{w}=E_{w} \cdot C_{g}=\frac{\rho g^{2} A^{2}}{4 \omega}
$$

where $E_{w}=\frac{1}{2} \rho g A^{2}$ is the wave energy, $A$ is the wave amplitude, and $C_{g}=\frac{g}{2 \omega}$ is group velocity in deep water. The unit of $P_{w}$ is Watts $/ \mathrm{m}$.

The power absorption performance of the device can be evaluated by the power capture width, according to the following equation

$$
C_{w i d t h}=\frac{\bar{P}}{P_{w}}=\frac{2 \omega^{3}}{\rho g^{2}} d_{i}\left|\frac{\xi_{i}}{A}\right|^{2}=\frac{2 \omega^{3}}{\rho g^{2}} d_{i}|\mathrm{RAO}|^{2}
$$

where $\omega$ is the frequency, $\rho$ is the water density, $g$ is the gravitational acceleration, $d_{i}$ is system damping coefficient corresponding to mode $i, \xi_{i}$ is the motion amplitude of the $i^{t h}$ hinge joint in waves. $\frac{\xi_{i}}{A}$ is defined as the response amplitude operator (RAO). The unit of $C_{w i d t h}$ is the meter.

\subsubsection{Power Absorption in Irregular Sea States}

A sea state can be characterized by two parameters: the significant wave height $H_{s}$ and the mean wave period $T_{p}$. The JONSWAP spectrum derives from the Joint North Sea Wave Project (Hasselmann et al., 1973) and constitutes a modification to the Pierson-Moskowitz spectrum to account for the regions that have geographical 
boundaries that limit the fetch in the wave generating area, e.g., the North Sea.

$$
\begin{gathered}
S_{A}(\omega)=\frac{5 H_{s}^{2} \omega_{p}^{4}}{16 \omega^{5}} \exp \left[-\frac{5}{4}\left(\frac{\omega_{p}}{\omega}\right)^{4}\right] \gamma^{a}(1-0.287 \ln \gamma) \\
a=\exp \left[-\frac{\left(\omega-\omega_{p}\right)^{2}}{2 \sigma^{2} \omega_{p}^{2}}\right] \\
\sigma=\left\{\begin{array}{cc}
0.07 & \text { when } \omega \leq \omega_{p} \\
0.09 & \text { when } \quad \omega>\omega_{p}
\end{array}\right.
\end{gathered}
$$

where $\omega_{p}=2 \pi / T_{p}$ is the modal frequency corresponding to the highest peak of the spectrum, in radians/sec, $H_{s}$ is the significant wave height in meters, $\gamma$ is the peakedness parameter from $1-7$, with a mean value of 3.3 .

In irregular sea states, the amplitude of each component of the irregular wave is formulated by

$$
A(\omega)=\sqrt{2 S_{A}(\omega) d \omega}
$$

where $S_{A}$ is the wave spectrum, $A$ is the wave amplitude. Correspondingly, the response amplitude is represented by the response spectrum

$$
\xi(\omega)=\sqrt{2 S_{\xi}(\omega) d \omega}
$$

The relation between response spectrum and wave spectrum is

$$
S_{\xi}(\omega)=S_{A}(\omega)|\operatorname{RAO}(\omega)|^{2}
$$

Substitute equation 3.49 and 3.50 into equation 3.42, then the average power absorp- 
tion in an irregular sea state is

$$
P_{a b s}=\int_{0}^{\infty} d_{i} \omega^{2} S_{A}(\omega)|\operatorname{RAO}(\omega)|^{2} d \omega
$$

The available wave power of a irregular sea state is computed by substituting $A^{2}=$ $2 S_{A}(\omega)$ into equation 3.43 that the overall available power for each sea state is

$$
P_{\text {avai }}(\omega)=L \int_{0}^{\infty} \rho g c_{g}(\omega) S_{A}(\omega) d \omega
$$

where $L$ is the length of the structure, $c_{g}$ is the group velocity that in deep water $c_{g}=\frac{g}{2 \omega}, S_{\xi}(\omega)$ is the wave spectral density. The power absorbed proportion is

$$
\text { prop. }=\frac{P_{a b s}}{P_{a v a i}}
$$

\subsubsection{Power Absorption at a Certain Location}

In a chosen operation site, according to the annual wave climate, the total absorbed power is the summation of the average power of every single sea state weighted by their probability of occurrence:

$$
P_{\text {annual }}=\sum_{k=1}^{N} \eta_{k} P_{a b s_{k}}
$$

where $P_{\text {annual }}$ is the total annual absorbed power, $\eta_{k}$ is the probability factor of a sea state, $P_{a b s_{k}}$ is the absorbed power for the $k^{t h}$ sea state.

\subsubsection{Power Absorption of SeaWEED}

In Figure 3.5, (b) and (c) illustrate two hinge modes of SeaWEED with the elevation at PTO joint 1 and PTO joint 2. The numbering of the joints is from stern to bow. 


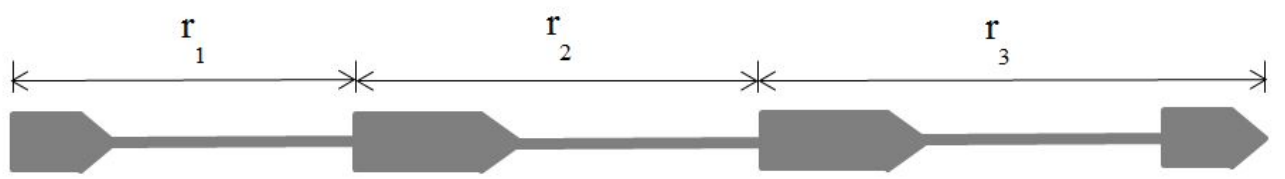

(a)

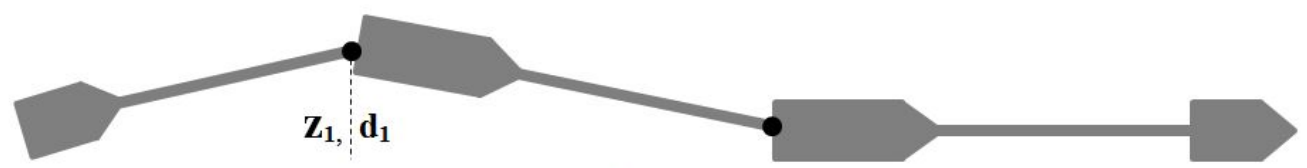

(b)

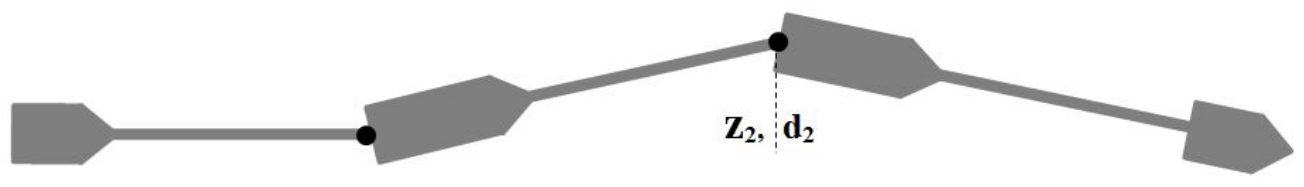

(c)

Figure 3.5: Hinge modes of SeaWEED illustrated in angular motions.

The vertical elevation amplitudes at each joint are $z_{1}$ and $z_{2}$ respectively. $r_{1}, r_{2}$ and $r_{3}$ are lengths of the three hinged segments of SeaWEED.

The motion of SeaWEED WEC in waves is computed by WAMIT. The hinge motion in WAMIT is considered as the vertical elevation at the hinge joint. Thus, the PTO damping parameters at the hinge joints are translational, in the unit of $\mathrm{N} \cdot \mathrm{s} / \mathrm{m}$.

Therefore, the average power absorption equation containing translational damping terms $d_{1}$ and $d_{2}$ is calculated as

$$
\Sigma \bar{P}=\frac{1}{2} \omega^{2}\left(d_{1} z_{1}^{2}+d_{2} z_{2}^{2}\right)
$$

In irregular seas, according to equation 3.51 the average power absorption of a Sea- 
WEED device can be calculated by

$$
\Sigma P_{a b s}=\int_{0}^{\infty} \omega^{2} S_{A}(\omega)\left(d_{1} z_{1}^{2}+d_{2} z_{2}^{2}\right) / A^{2} d \omega
$$

where $S_{A}(\omega)$ is the wave spectrum density at each frequency component, $A$ is the wave amplitude. In the numerical study, PTO damping parameters are set to be equal at two joints. 


\section{Chapter 4}

\section{Model Test and Frequency Domain}

\section{Simulation}

\subsection{Model Test of the First Generation Seaweed}

\subsubsection{Overview of the Model Test}

The one-sixteen scale model test of the first generation SeaWEED model was conducted by the Grey Island Energy (GIE) Incorporation. The total assembled device consists of four hulls and two PTO joints.

The first generation SeaWEED model was tested in Offshore Engineering Basin (OEB) at NRC-IOT located in St. John's, NL. The OEB is a large tank with dimensions of $75 \mathrm{~m} \times 32 \mathrm{~m} \times 3.2 \mathrm{~m}$. The model test followed the Marine Renewable Energy Guide for Tank Testing provided by European Marine Energy Centre (EMEC) (Holmes, 2009). For the experiments conducted, the following instruments were calibrated and utilized: linear transducer and pressure sensor onboard the third module along with a pressure sensor and flow meter on the test bed. A prototype of the hydraulic PTO system was built. Water flows from the SeaWEED model through hydraulic lines where pressure 
is built up to a desired level in an accumulator. The power was calculated by the product of hydraulic pressure and flow rate.

The 1:16 model was tested with a head sea direction in monochromatic waves. After all the systems and measuring components were installed during the tank test, the draft was over the midsection of the body, as shown in Figure 4.1, which was higher than planned. The parameters of the 1:16 model and full scale model are shown in Table 4.1.

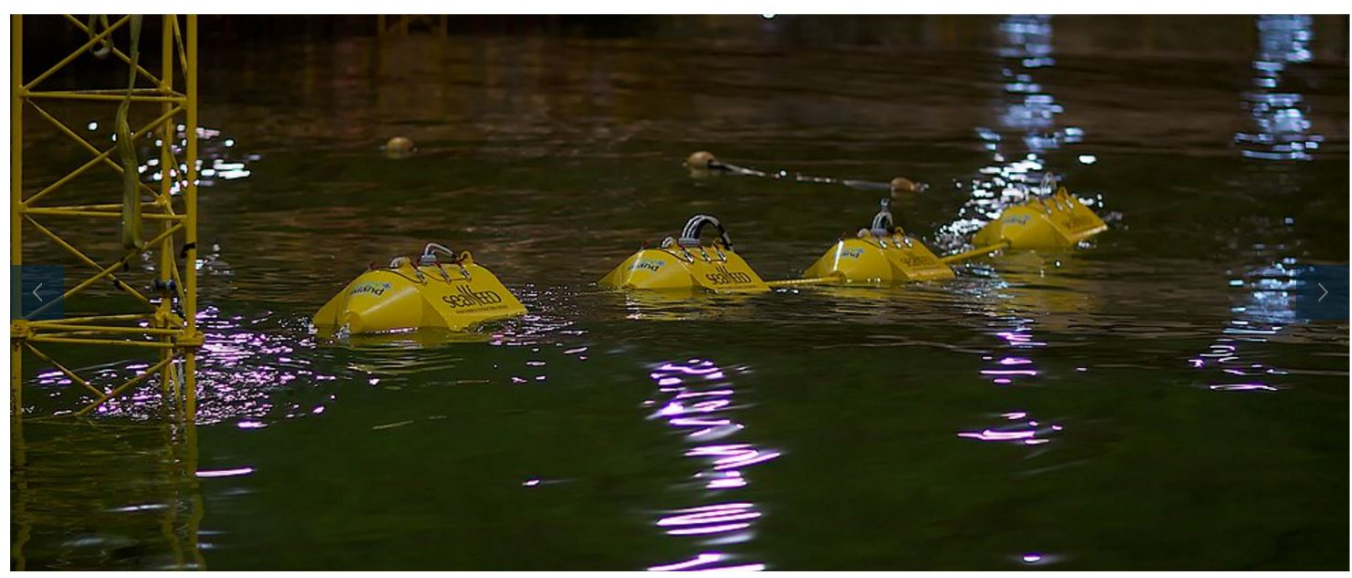

Figure 4.1: The 1:16 model of the first generation SeaWEED in wave tank.

Table 4.1: Parameters of the first generation SeaWEED model.

\begin{tabular}{ccc}
\hline Parameters & 1:16 model scale $(\mathrm{m})$ & Full scale $(\mathrm{m})$ \\
\hline Total length & 9.04 & 145.0 \\
Module length & 1.0 & 16.0 \\
Tie-rod length & 1.68 & 27.0 \\
Beam & 0.55 & 8.80 \\
Height & 0.43 & 6.88 \\
Planned draft & 0.16 & 2.5 \\
Model test draft & 0.28 & 4.48 \\
\hline
\end{tabular}




\subsubsection{Comparison between Experimental and Numerical Re- sults}

The one-sixteenth scale model test was conducted for four wave periods, which are $6 \mathrm{~s}, 8 \mathrm{~s}, 9 \mathrm{~s}$ and $10 \mathrm{~s}$ in full scale. The results of the model test were compared with the numerical solutions in frequency domain. The experimental power absorption was obtained from the pressure and the flow rate in the hydraulic system. Since no generator was equipped for this round of testing, there is no upper limit of the generator capacity, i.e., the power absorption results were not constrained with a upper bound.

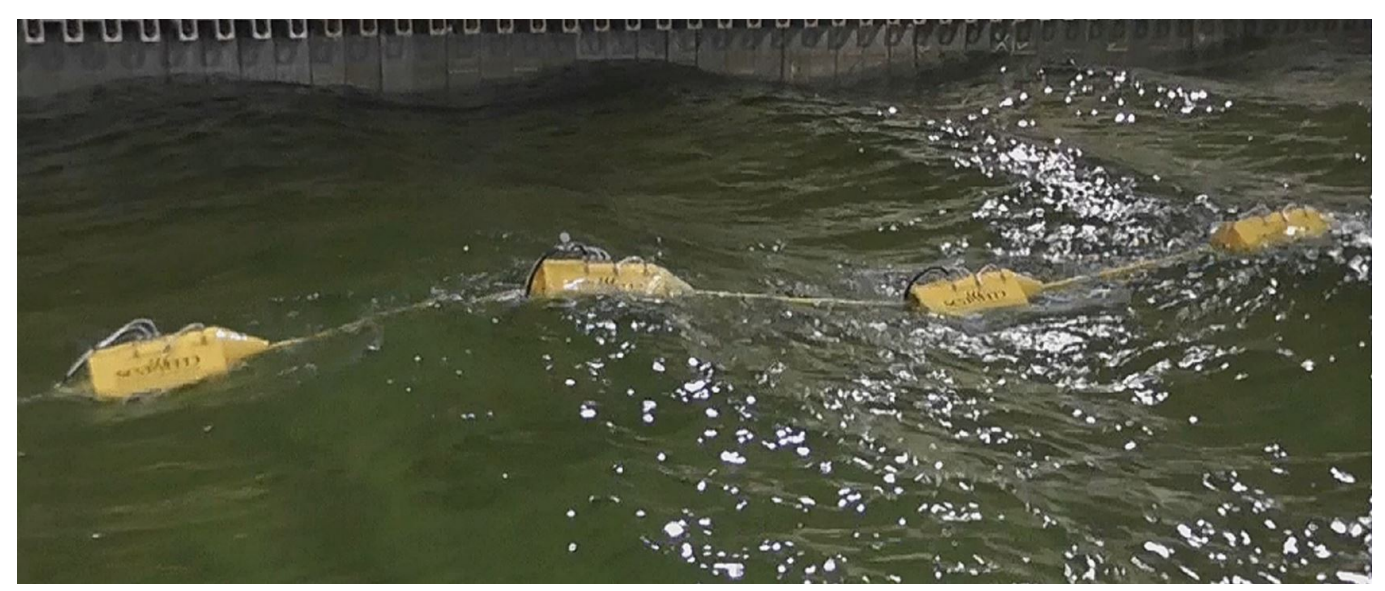

Figure 4.2: The 1:16 model of the first generation SeaWEED in waves.

As shown in Figure 4.2, during the model test, the incoming waves go through the hinged array and the model responses with joint elevation. In numerical simulation, the SeaWEED device was considered as three rigid bodies connected by two hinged joints that allow their relative motions. The waves go through the body leading the vertical displacements at the joints. As only the relative motions between two adjacent bodies are intended for power generation, studies have been focused on the vertical motion at two hinge joints. The vertical elevations at joint 1 and joint 2 were computed by using WAMIT, and the PTO damping was calculated from the total 
power loss between unconstrained motion power and absorbed power.

Figure $4.3,4.4$, and 4.5 presented the comparison of power absorption between tank test and numerical simulation at the wave heights of 3,4 , and 5 meters in full scale. The curves are generated by numerical computation and the points are resulted from the mode test. It is shown that at a wave height of 2 meters, the numerical results match perfectly with the tank test results, while at 6 seconds and 12 seconds, the tank test results are slightly higher than the numerical results. When the wave height goes higher, the linear assumption might not be applicable. Therefore, in the real test, the device cannot response ideally to the wave, and the elevation amplitude will be lower than the ideal numerical solution which is computed based on the linear assumption. This explains why the numerical results become higher than the tank test when the wave height exceeds 4 meters. From the figures, it is apparent that both the curves and tank test points reach the maximum power absorption at 8.5 seconds of wave period. The trends are consistent with each other. The comparison validated the numerical approach that was applied in this thesis.

\subsubsection{Improvements}

During the model test, problems have been found and improvements were made for the design of the second generation model. The draft of the first generation model during the test was deeper than planned. Also, the tie-rod experienced deflection during certain wave conditions. Improvements were made on the hull geometry and connection structure for the second generation model. The new design of the flat hull geometry was aimed to simplify the manufacture process that reduce the manufacturing time and cost. Also the interconnecting structure was changed to truss which has more strength.

The numerical approach was validated by comparing to the experimental results of 


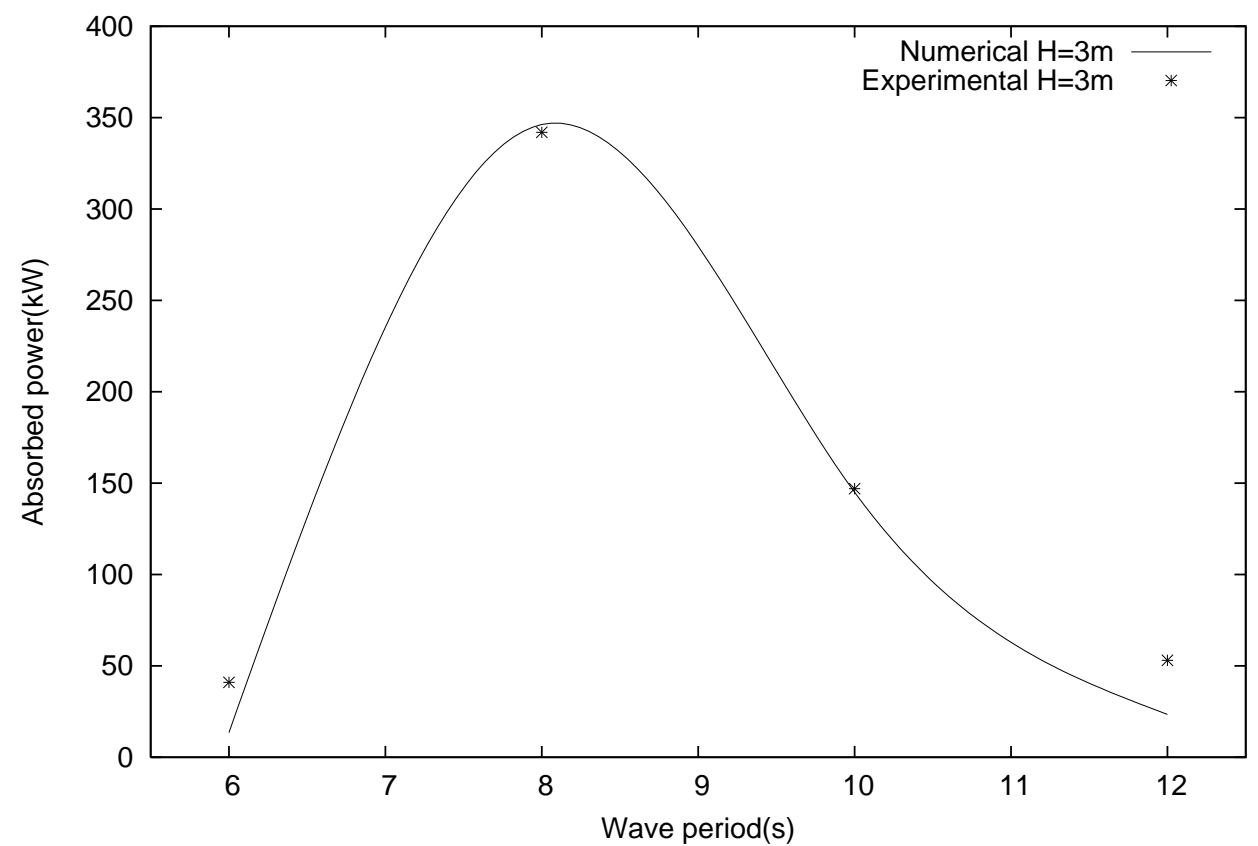

Figure 4.3: Comparison of the absorbed power between model test and numerical simulation for the first generation of SeaWEED model at a wave height of 3 meters.

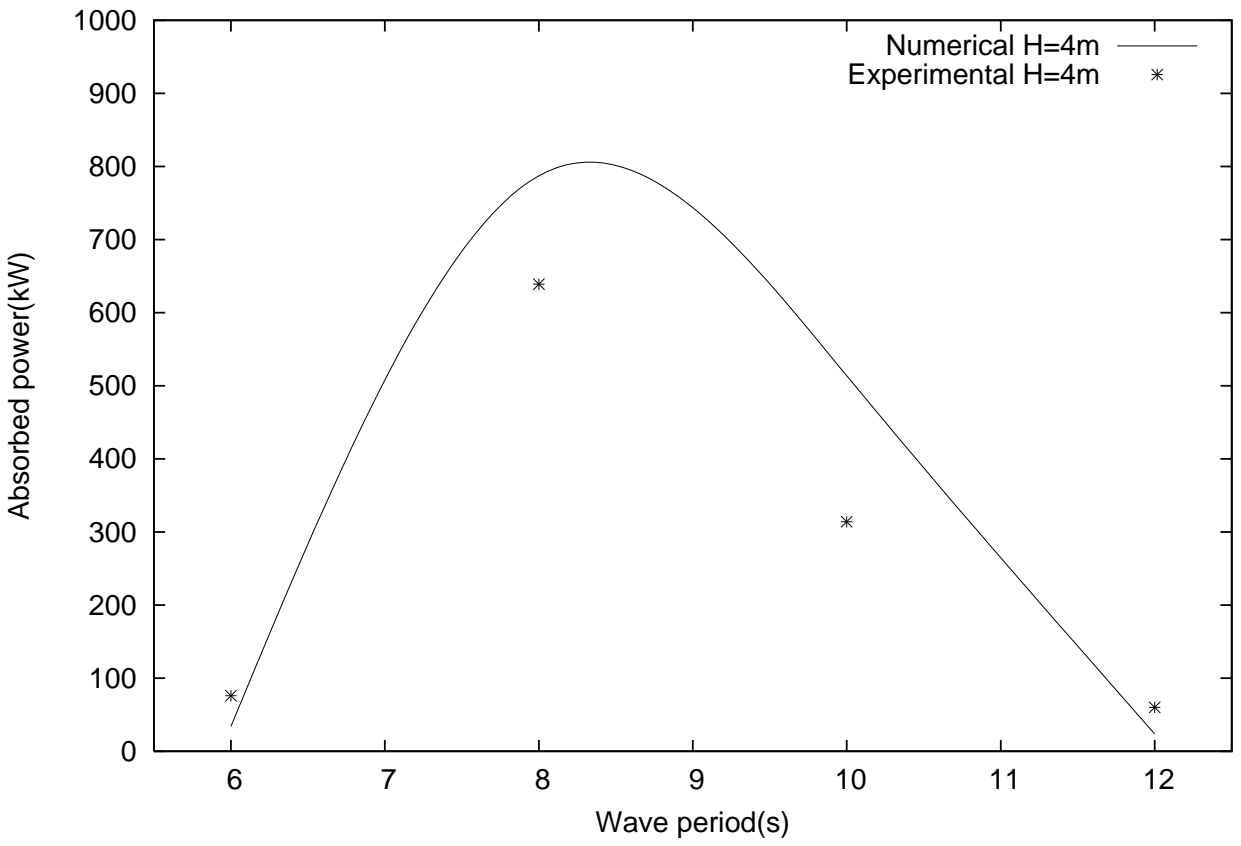

Figure 4.4: Comparison of the absorbed power between model test and numerical simulation for the first generation of SeaWEED model at a wave height of 4 meters. 


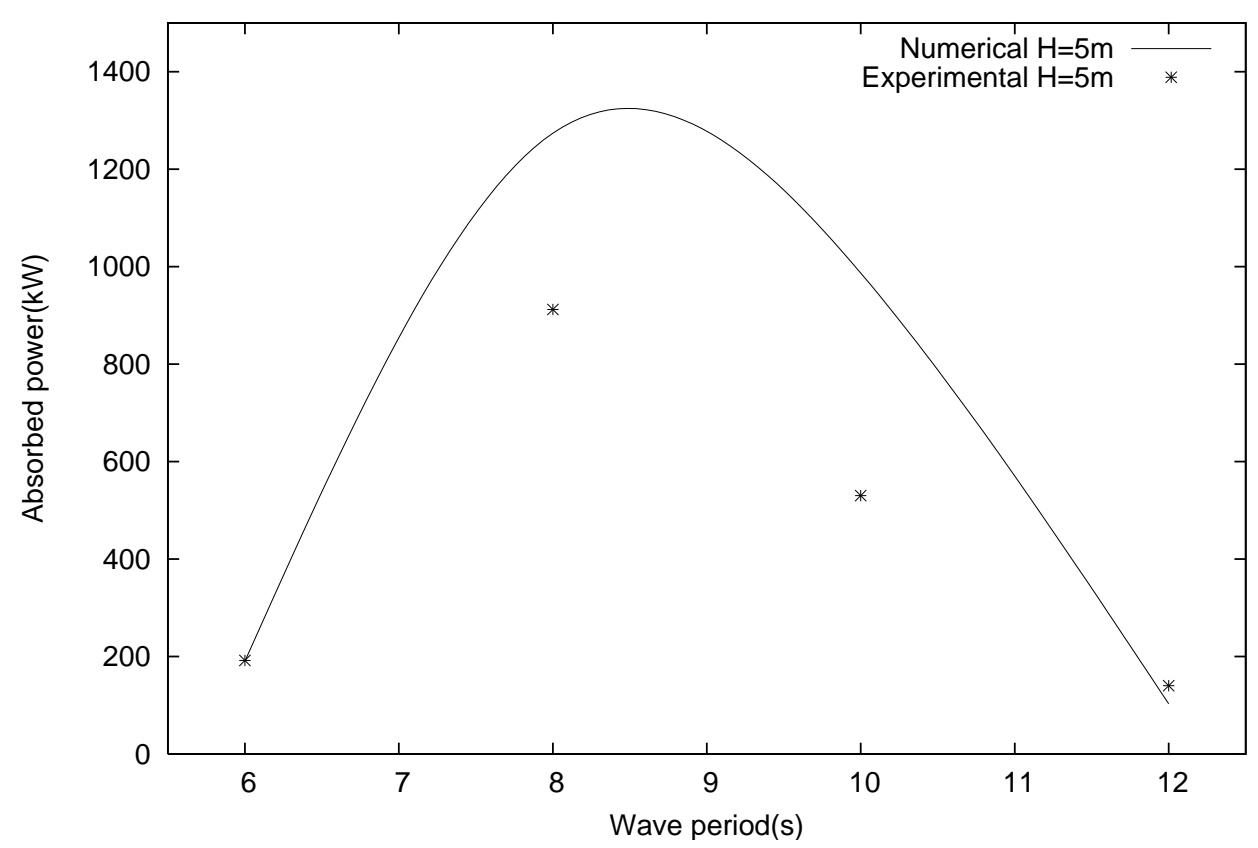

Figure 4.5: Comparison of the absorbed power between model test and numerical simulation for the first generation of SeaWEED model at a wave height of 5 meters.

the first generation model. Before it was applied to analyze and optimize the second generation of SeaWEED model, a verification study was performed to verify the preprocessing mass matrix code and a convergence study was performed to determine the appropriate panel number for the numerical simulation.

\subsection{Frequency Domain Simulation on the Second Generation of SeaWEED}

As shown in Figure 4.6, the SeaWEED wave energy converter consists of four hulls (a nose, the first producing unit, the second producing unit and a tail) and three connecting trusses. The initial designed principal parameters of a SeaWEED device 
Table 4.2: Initially designed principal parameters of SeaWEED in full scale.

\begin{tabular}{cc}
\hline Parameters & The second generation SeaWEED 1:1 Model \\
\hline Total length $(\mathrm{m})$ & 140.0 \\
Beam $(\mathrm{m})$ & 8.0 \\
Height $(\mathrm{m})$ & 5.0 \\
Design draft $(\mathrm{m})$ & 1.5 \\
Length of truss $(\mathrm{m})$ & 30.0 \\
Length of producing units $(\mathrm{m})$ & 16.0 \\
Length of nose and tail units $(\mathrm{m})$ & 9.0 \\
Number of PTO joints & Two \\
Initial PTO damping $(\mathrm{N} \cdot \mathrm{s} / \mathrm{m})$ & $7 \times 10^{5}$ \\
\hline
\end{tabular}

are given in Table 4.2, where the connecting truss length is 30 meters.

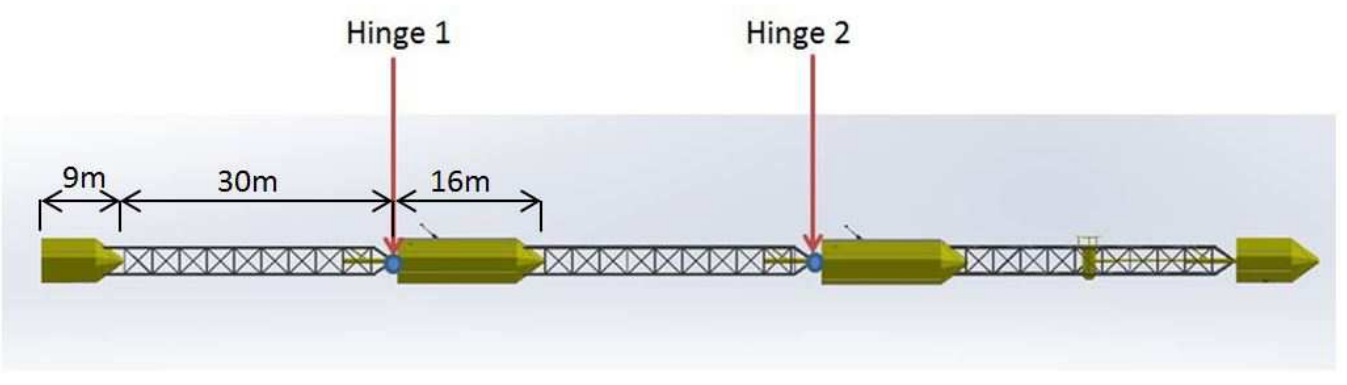

Figure 4.6: Dimension of Initial Designed SeaWEED wave energy converter.

In numerical simulation, the SeaWEED is considered as three rigid segments connected by two hinged joints that allow their relative motions. The three segments are in different lengths, as given in Table 4.3. 
Table 4.3: Lengths of three segments for the initially designed SeaWEED, from stern to bow.

\begin{tabular}{ccc}
\hline Segment No. & Elements included & Length $(\mathrm{m})$ \\
\hline 1 & Tail unit and connecting truss & 39 \\
2 & The first producing unit and connecting truss & 46 \\
3 & The second producing unit, connecting truss and nose unit & 55 \\
\hline
\end{tabular}

\subsubsection{Convergence Study}

The motion of SeaWEED was computed by WAMIT. WAMIT is a radiation/diffraction program based on a three-dimensional panel method and potential theory (Lee and Newman, 2013). For motions of hinged vessels, WAMIT permits the analysis of 'generalized modes' for hinge motions in addition to the six rigid conventional motions. N-body hinged array will have six conventional modes and $N-1$ hinge modes (Newman, 1997) and the indices of hinge modes are defined from index 7 to $N+5$. In WAMIT, the multiple bodies of a hinge array will be considered as one body, i.e. NBODY $=1$. The body is sub-divided into separate hinged segments, to permit the analysis of a hinged structure using generalized modes. The mass matrix of SeaWEED was calculated by pre-processing code and was input into WAMIT.

The numerical model of SeaWEED was built by 3-D panels. A convergence study was performed to determine the best panel numbers of computing. Figure 4.7 shows the relation between panel numbers and the corresponding residuals, by setting the results from a panel number of $10^{5}$ as the reference. The RAOs of free hinged SeaWEED at six wave periods were computed and the mean squared error (MSE) was used to evaluate the accuracy of the results. Table 4.4 shows the number of panels and the corresponding mean squared errors. Due to the capacity of the computer, a panel number of 4096 was chosen for the following computations. The same panel number was used in the numerical simulation of the first generation SeaWEED model. 
Table 4.4: Convergence study on the panel numbers.

\begin{tabular}{ccc}
\hline Case No. & Panel Numbers & Mean Square Error \\
\hline 1 & 488 & 0.00546 \\
2 & 1104 & 0.00121 \\
3 & 3760 & 0.00035 \\
4 & 4200 & 0.00003 \\
5 & 7328 & 0.00001 \\
6 & 10000 & 0.00000 \\
\hline
\end{tabular}

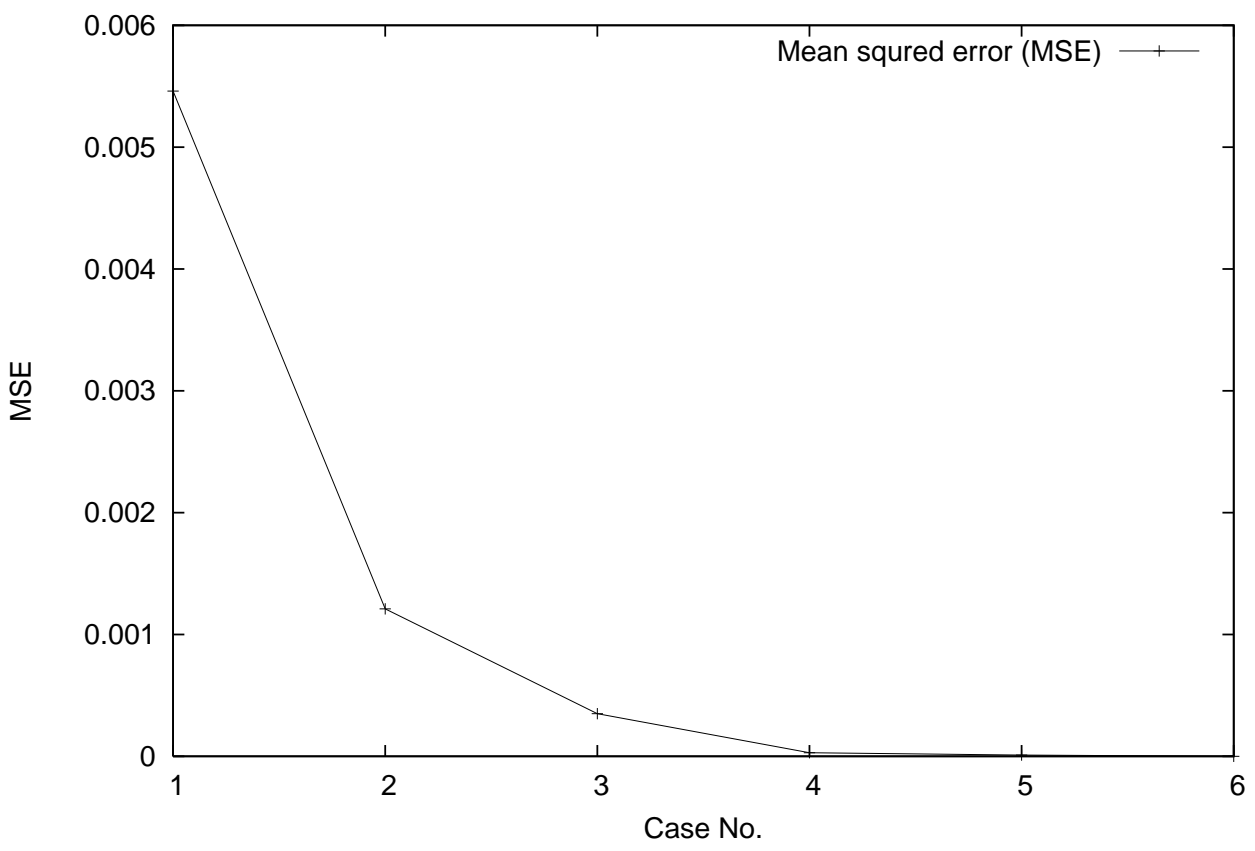

Figure 4.7: Convergence study on the panel numbers to model SeaWEED in WAMIT. 


\subsubsection{Motion of the Second Generation of SeaWEED in Waves}

In this section, hinge motions of SeaWEED were computed with and without considering the PTO constraints to the joints. The simulated wave condition is:

- Wave depth: 50 meters

- Wave heading: 180 degree head-sea

- Wave period: 1.0 to 18.5 seconds

Unrestrained free hinge motions with no PTO effect were presented in figure 4.8. The RAO curves present the vertical elevations at hinge joints that caused by relative pitch motions between two adjacent bodies. It is shown that from 1.0 to 18.5 seconds, both curves have three peaks while the main peaks both occur at the period of 9 seconds. At the periods of 4.5 seconds and 6.5 seconds, there are two side peaks of elevation at two hinge joints. The side peaks of hinge joint 2 are more significant than of hinge joint 1 . It is caused by the interaction between the three hinged segments. The lengths and mass distributions of the segments are different so that each segment has a different natural frequencies. After 7.5 seconds, both curves go smoothly; the vertical elevations of the hinge joints reach the maximum RAOs of 2.12 and 2.00 respectively at 9 seconds.

An initial value of external damping at $7 \times 10^{5} \mathrm{~N} \cdot \mathrm{s} / \mathrm{m}$ was added to the hinge joints to simulate the PTO effect. Figure 4.9 presents the damped hinge motion at two PTO joints. It is shown that the restrained RAO curves are smoother and reach the maximum response amplitudes of 0.95 and 0.8 respectively at the period of 9.5 seconds.

Considering the wave condition of offshore areas, the wave periods of from 5.5 seconds to 12 seconds were the range of interest. Figure 4.10 presents the restrained 


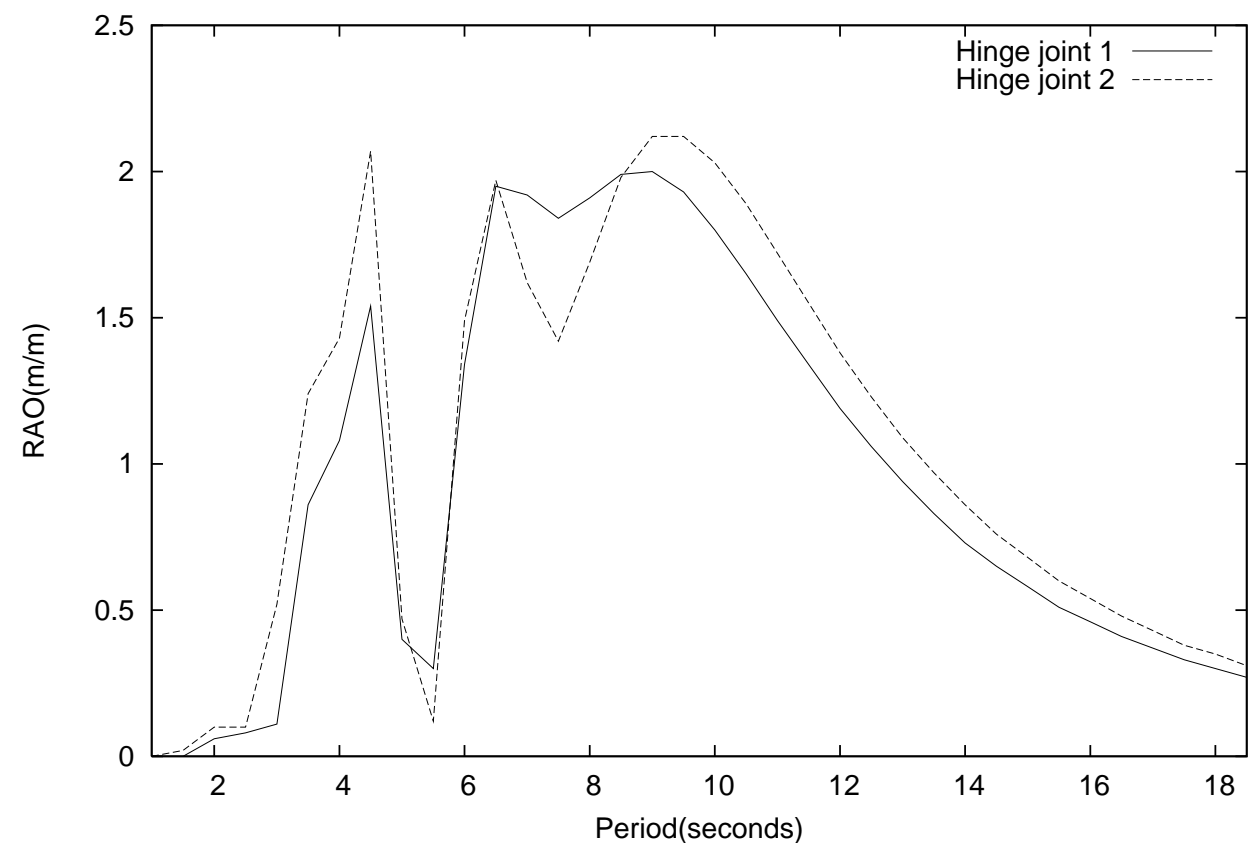

Figure 4.8: Free hinge motion of SeaWEED.

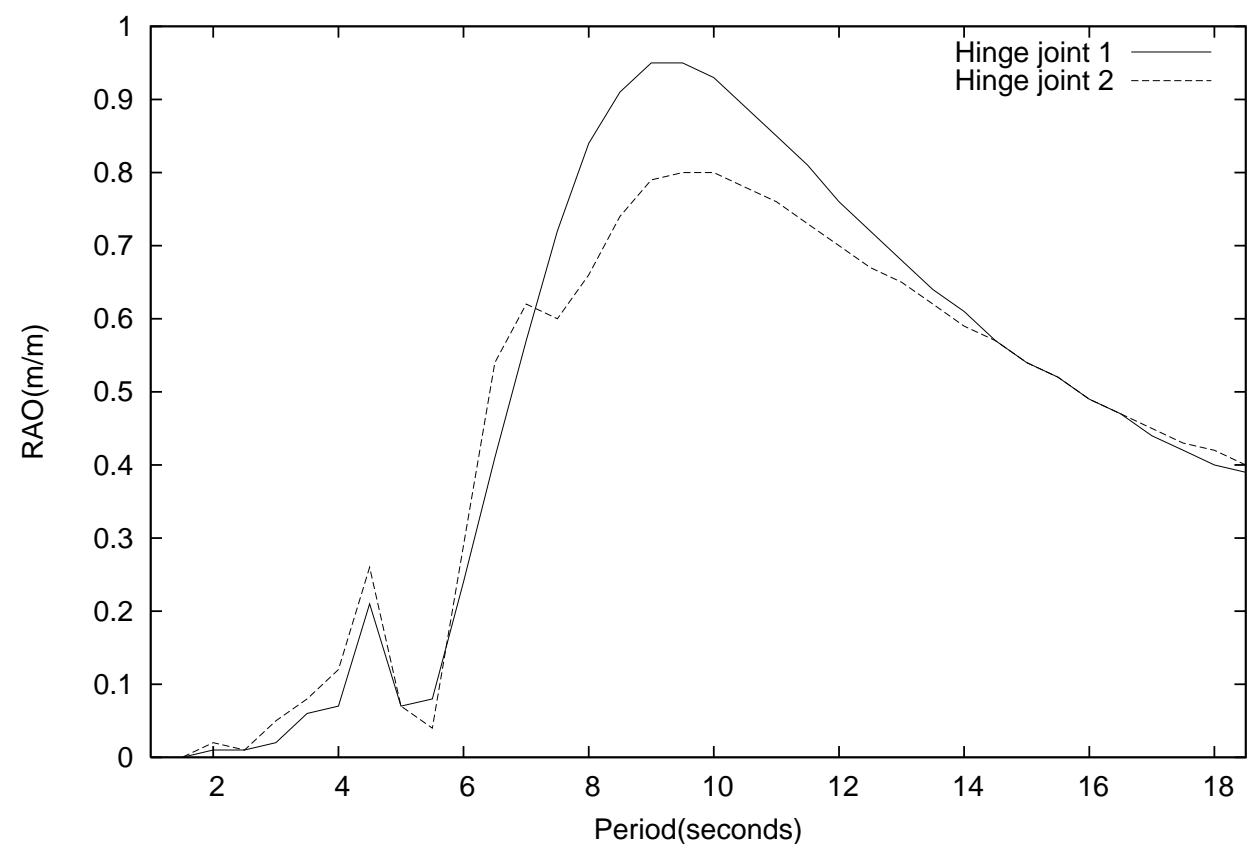

Figure 4.9: Restrained hinge motion of SeaWEED with a $7 \times 10^{5} \mathrm{~N} \cdot \mathrm{s} / \mathrm{m}$ PTO damping. 
hinge motion with a $7 \times 10^{5} \mathrm{~N} \cdot \mathrm{s} / \mathrm{m}$ PTO damping within 5.5 to 12 seconds; Figure 4.11 presents the corresponding power extraction width. The curve of power capture width reaches the maximum at 8.5 seconds. Therefore, it is shown that the primarily designed SeaWEED will have its best power absorption performance in a wave period of 8.5 seconds.

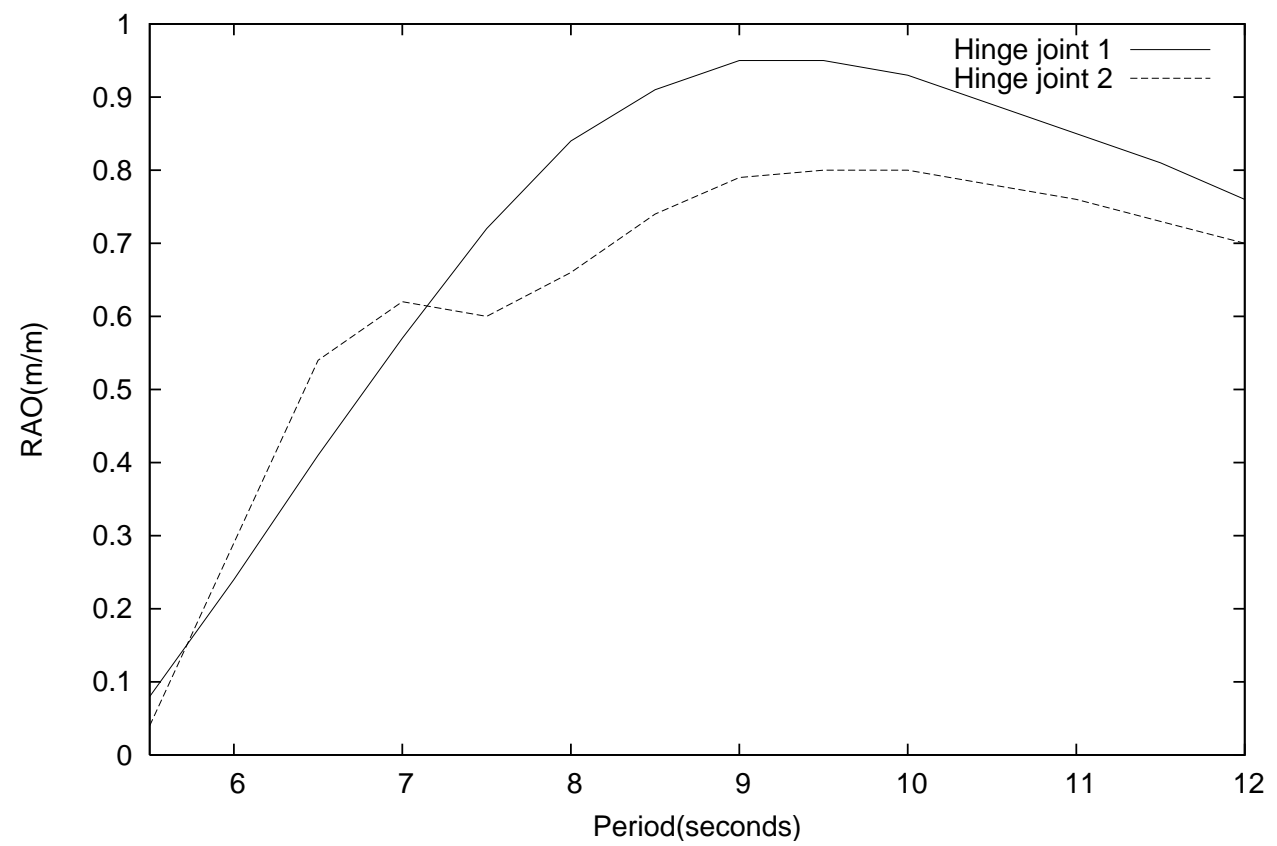

Figure 4.10: Restrained hinge motion of SeaWEED with a $7 \times 10^{5} \mathrm{~N} \cdot \mathrm{s} / \mathrm{m}$ PTO damping within 5.5 to 12 seconds.

In the next chapter, the damping value will be optimized to achieve a better power extraction width curve. Meanwhile, the connecting truss length and the draft will also be tuned in regular waves and at an intended operation site. 


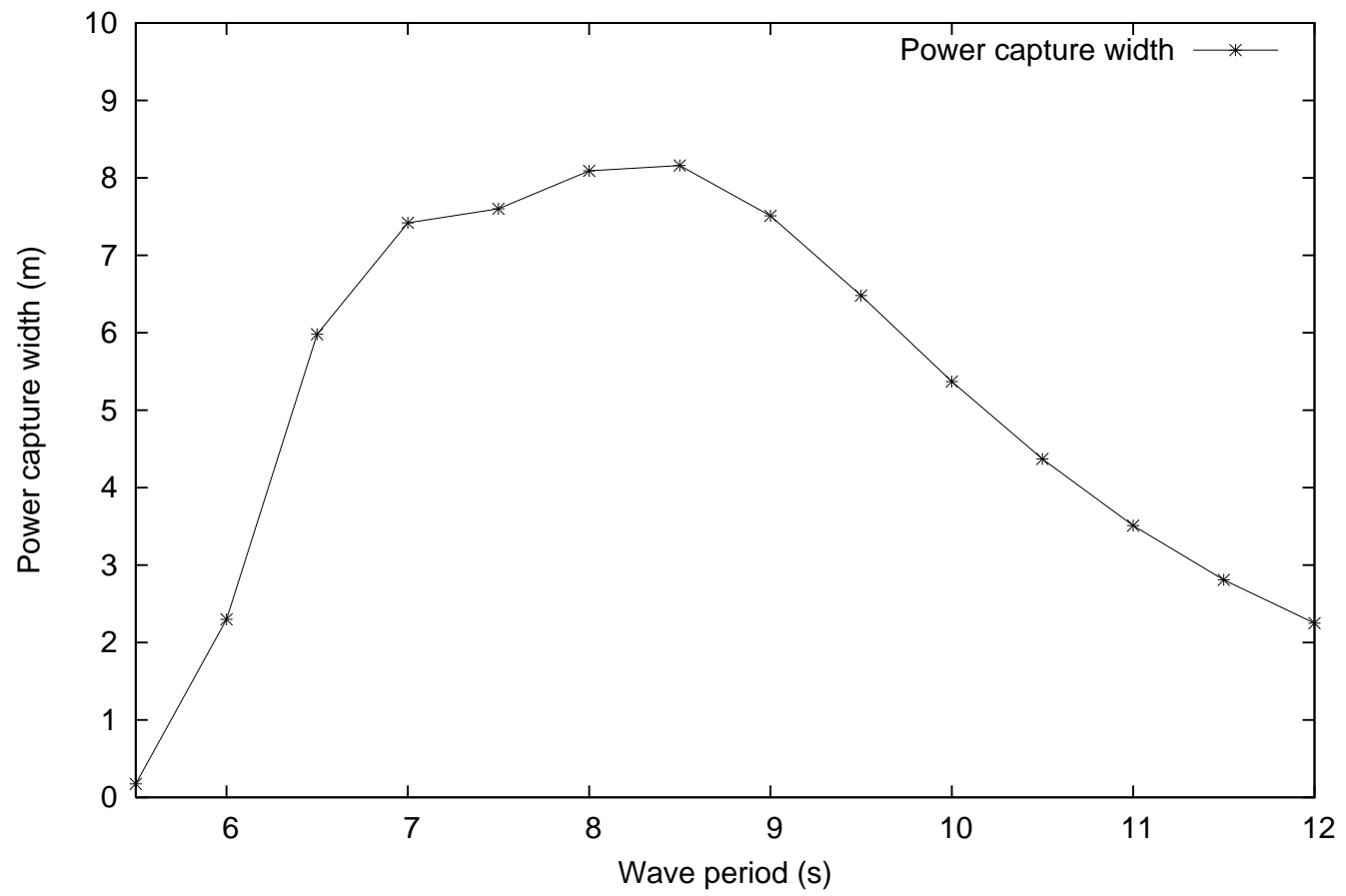

Figure 4.11: Power capture width of the primarily designed second generation of SeaWEED with a $7 \times 10^{5} \mathrm{~N} \cdot \mathrm{s} / \mathrm{m}$ PTO damping within 5.5 to 12 seconds. 


\section{Chapter 5}

\section{Numerical Optimization of the}

\section{Second Generation SeaWEED}

\section{Model}

\subsection{Design of the Optimization Experiments}

For different wave periods or different intended locations, the optimal length, draft and PTO parameter of SeaWEED might be varied. In this chapter, the optimization process involved geometrical parameters optimization and PTO optimization. The geometrical optimization variables are the truss length $L$, and the draft $T$. The PTO optimization variable is the damping $d$. In the SeaWEED PTO system, there is no restoring spring force in upper and lower hydraulic rams thus no external stiffness value was added. For the optimization in regular waves, the response is the power

extraction width integration $\int_{T_{-}}^{T_{+}} C_{\text {width }} d T$, where $T_{-}$and $T_{+}$are the lower and upper bounds of wave periods. No constraints were set for the optimization in regular waves. For the optimization at an intended site, the response is the annual power absorption 
and the results were calculated based on the response amplitudes at different wave heights; the constraints are:

- The angular movements of the PTO joints cannot exceed 30 degrees about its equilibrium position.

- The absorbed power cannot exceed $750 \mathrm{~kW}$ due to the capacity of the generator.

Response surface method was employed to optimize the power absorption performance by finding the best PTO damping and the best combination of truss length and draft of SeaWEED. Details on the response surface method can be found in the work of Myers and Montgomery (Myers et al., 2009). Software of Design-Expert with statistical and visual tools for design of experiments (DOE) was adopted to analyze the simulation results and to build the mathematical model which predicts the interaction between variables and responses and further provide the estimated mathematical optimization solutions.

The optimization process, assisted by using Design-Expert, is summarized as follows. Firstly, a group of rough simulation experiments were performed in order to determine the range of variables. After that, samplings were planned by full factorial design (Ryan and Morgan, 2007) or uniform design (Fang et al., 2000) within the determined space. Simulations were conducted in WAMIT and the data were post-processed by Fortran codes. Then the results were imported into Design-Expert and a best fitting model was chosen to fit the relation between $L, T, d$ variables and the response by polynomial regression. The optimal solutions were predicted according to the polynomial model. 


\subsection{Numerical Optimization in Regular Waves}

\subsubsection{Optimization of PTO Parameters}

The PTO damping was optimized by using Design-Expert. Based on the initial study, the damping values at two joints were regarded as equal and were set to be from $1 \times 10^{5} \mathrm{~N} \cdot \mathrm{s} / \mathrm{m}$ to $1 \times 10^{6} \mathrm{~N} \cdot \mathrm{s} / \mathrm{m}$ for the optimization. A sampling plan of 13 runs was designed. The objective was to maximize the power capture width within the wave period of 5.5 seconds to 12 seconds. The integration area of the power capture width

curve wave periods was computed by $\int_{T_{-}}^{T_{+}} C_{\text {width }} d T$, where $T_{-}$and $T_{+}$are the lower and upper bounds of wave periods, in order to evaluate the power absorption performance of the device in regular waves. The relation curve between the PTO damping and the integration of power capture width was fitted by polynomial regression method in Design-Expert, as shown in Figure 5.1.

A sixth-order nonlinear model was suggested with $99.95 \%$ accuracy by the analysis of Design-Expert to fit the relation between the variable and the response. It can be observed that for initial designed model, a PTO damping of $322500 \mathrm{~N} \cdot \mathrm{s} / \mathrm{m}$ is the best for achieving the optimal power absorption performance among 5.5 to 12 seconds of wave period. The predicted power capture width integration is $44.86 \mathrm{~m} \cdot \mathrm{s}$ according to the numerical model of the curve.

A verification study was performed by setting the damping value at $322500 \mathrm{~N} \cdot \mathrm{s} / \mathrm{m}$; the computed power capture width integration was $44.82 \mathrm{~m} \cdot \mathrm{s}$, which verified the polynomial model and optimal solution by the Design-Expert. The optimized power capture width at a damping of $322500 \mathrm{~N} \cdot \mathrm{s} / \mathrm{m}$ was compared with the primary design at a $700000 \mathrm{~N} \cdot \mathrm{s} / \mathrm{m}$ damping, as shown in Figure 5.2. It is shown that the optimal power capture width curve is higher than the original width curve among 5.5 to 12 seconds. The optimal curve has a small peak at 7 seconds of wave period, which is 


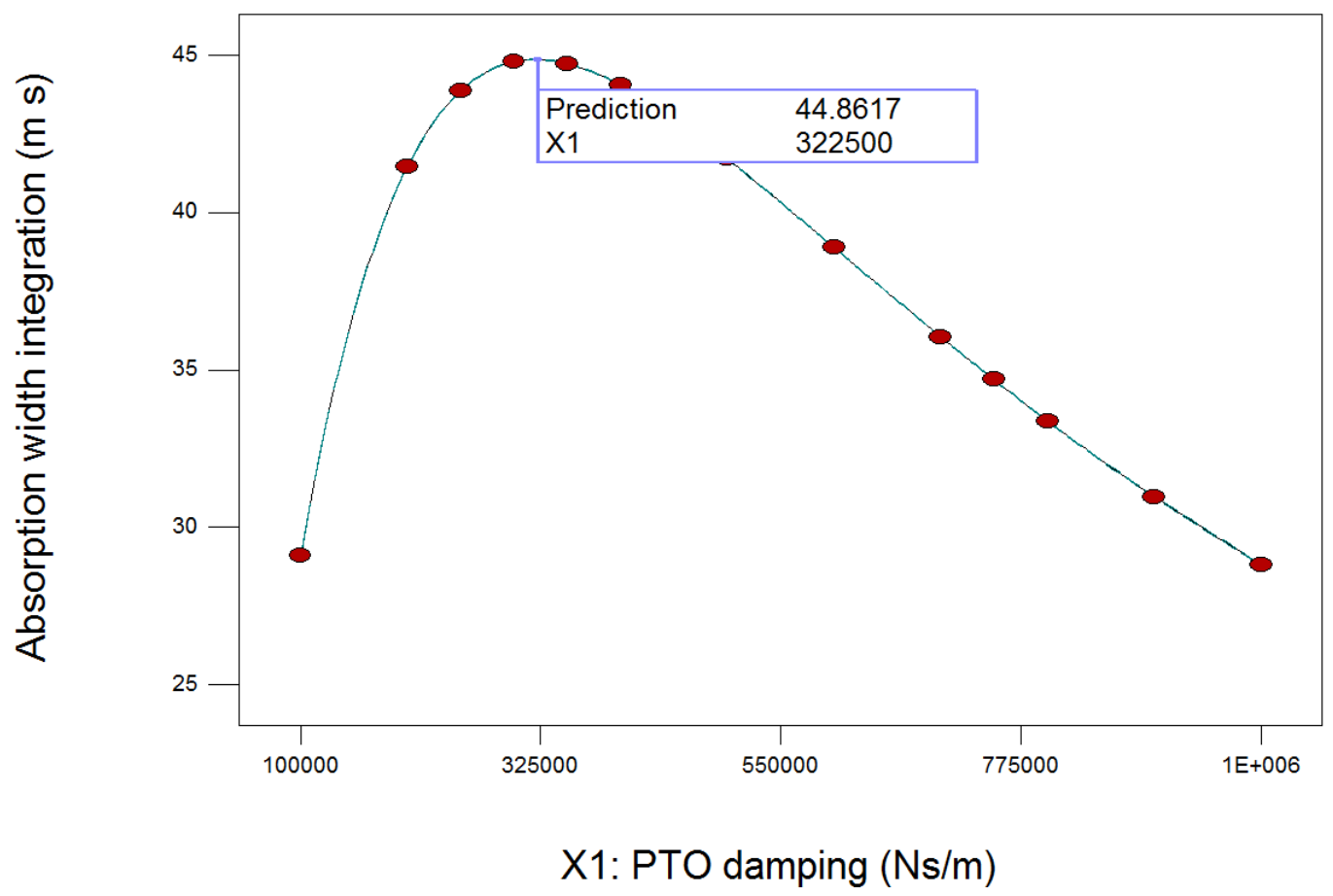

Figure 5.1: Response curve of the power capture(absorption) width integration with different damping values of the SeaWEED model with a 30-meter length of intermediate truss and a 1.5-meter draft. 
because that the hinge elevation at joint 2 is influenced by the coupling effect from the first joint, as shown in Figure 5.3.

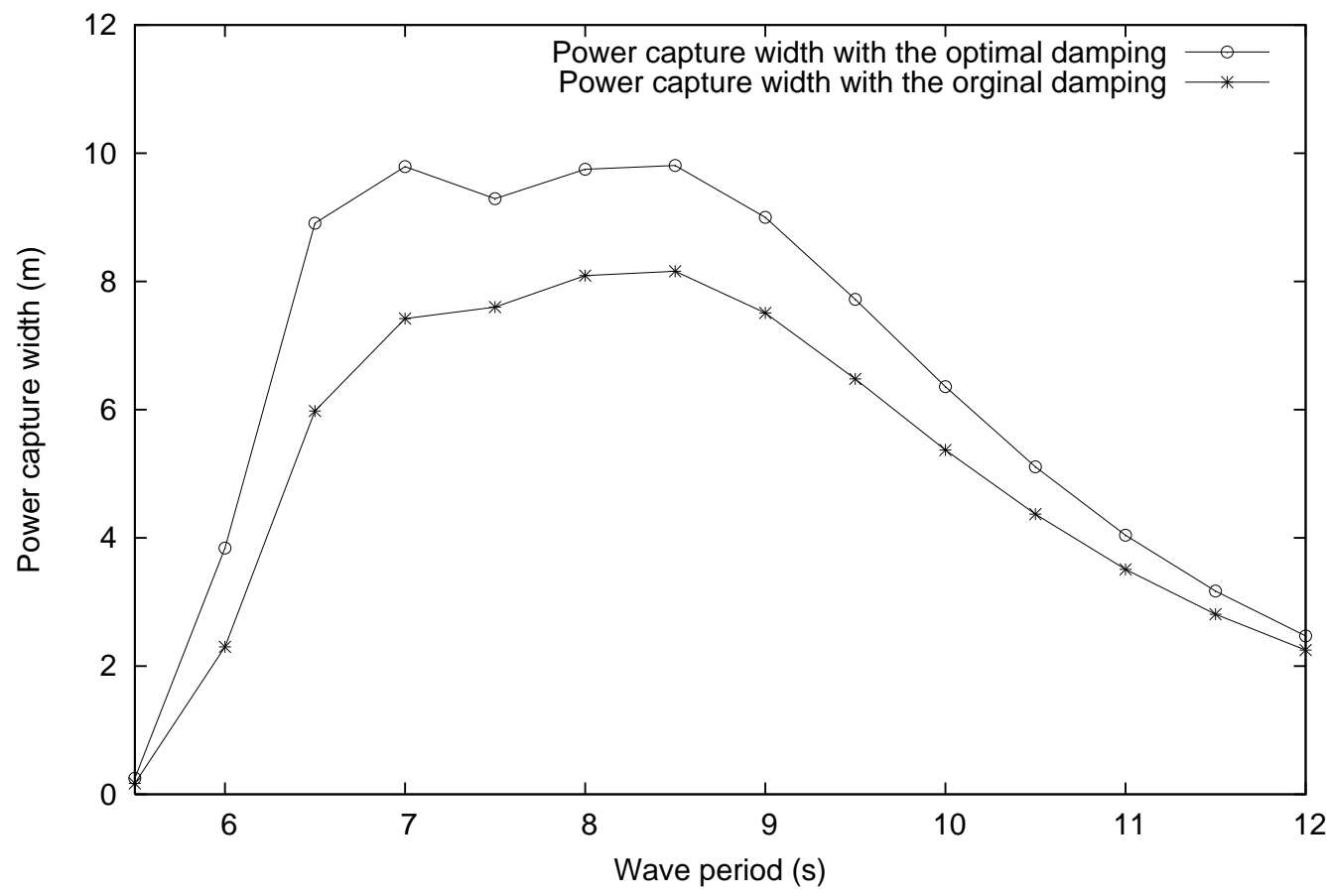

Figure 5.2: Power capture width comparison between original designed damping and optimal damping.

\subsubsection{Optimization of the Geometrical Parameters}

For different wave conditions, the optimal truss length and draft of the device can be varied. A full factorial sampling plan for a series of truss lengths and drafts was designed. Since the optimal value of PTO damping was influenced by the wetted surface and underwater geometry, it was not regarded as independent to the geometrical parameters. Thus a two-step optimization was performed. Each combination 


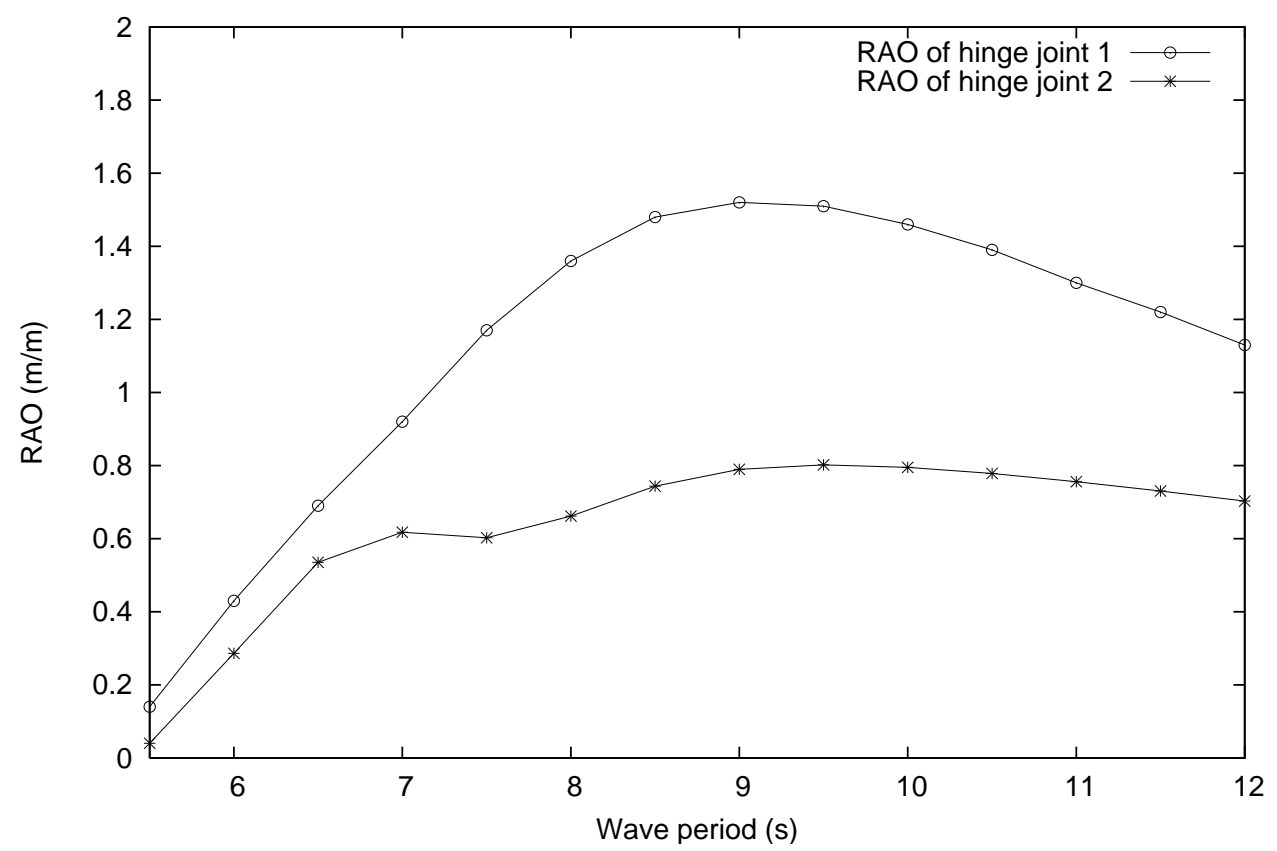

Figure 5.3: RAO curves at two hinge joints of the initial model with an optimal PTO damping.

of truss length and draft defined a model. For each model, the damping was tuned to the optimal value by repeating the PTO optimization process. Table 5.1 gives a description of the geometrical optimization design.

Table 5.1: Overview of the optimization design in regular waves.

\begin{tabular}{cccc}
\hline Variables & $\mathrm{L}(\mathrm{m})$ & $\mathrm{T}(\mathrm{m})$ & $\mathrm{d}(\mathrm{N} \cdot \mathrm{s} / \mathrm{m})$ \\
\hline Lower value & 10 & 1.5 & $10^{5}$ \\
Upper value & 100 & 4.0 & $10^{6}$ \\
Levels & 10 & 5 & 5 \\
Response & Capture width integration $(\mathrm{m} \cdot \mathrm{s})$ \\
\hline
\end{tabular}

For simulation based optimization, one of the advantages is the efficiency that the sampling space can be larger than in the physical experiment. By full factorial de- 
sign, a total number of 250 simulation cases were computed and the data were post processed by own codes. Response surfaces were generated by Design-Expert to find the optimal geometrical parameters at each wave period.

Figure 5.4 to 5.10 present the response surfaces of capture width integration with different truss length, drafts, and PTO damping at each wave period within 6 to 12 seconds. Each surface was generated at an optimal damping value shown on the left of the figure. Quartic polynomial models were suggested by Design-Expert to fit the relation between the variables and the response. The contours represent the nondimensional response from 0 to 1 . The flag with ' 1 ' indicates the location of optimal truss length and draft to achieve a maximum power capture integration. For example in Figure 5.10, when the wave period is 12 seconds, the predicted optimal truss length is 62.3 meters and the draft is 2.2 meters. For a quartic model, sometimes there is more than one optimal solution. Considering the cost and structure strength, the solution with minimum truss length and draft was chosen.
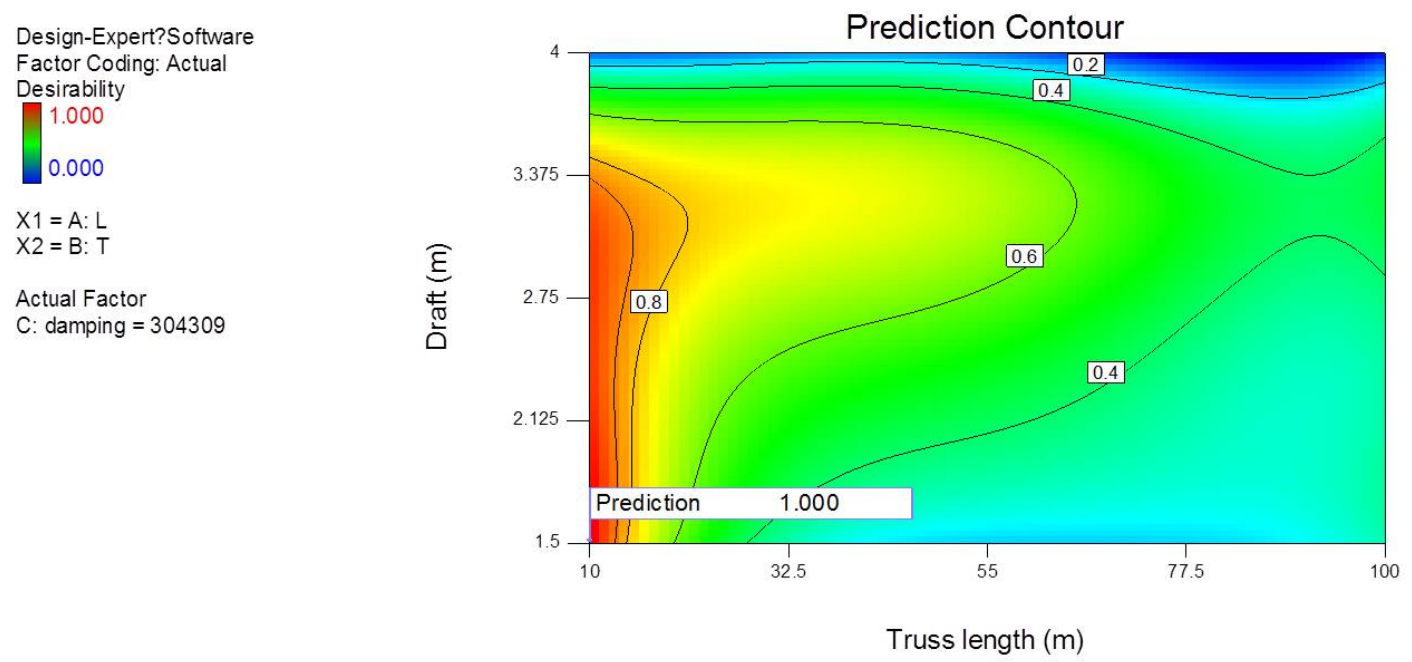

Figure 5.4: Response surface of the power capture width integration in regular waves with a wave period at 6 seconds.

A summary of optimization solutions for each wave period is shown in Table 5.2. The 
Design-Expert?Software

Factor Coding: Actual

Desirability

1.000

0.000

$\mathrm{X} 1=\mathrm{A}: \mathrm{L}$

$X 2=B: T$

Actual Factor

C: damping $=304764$

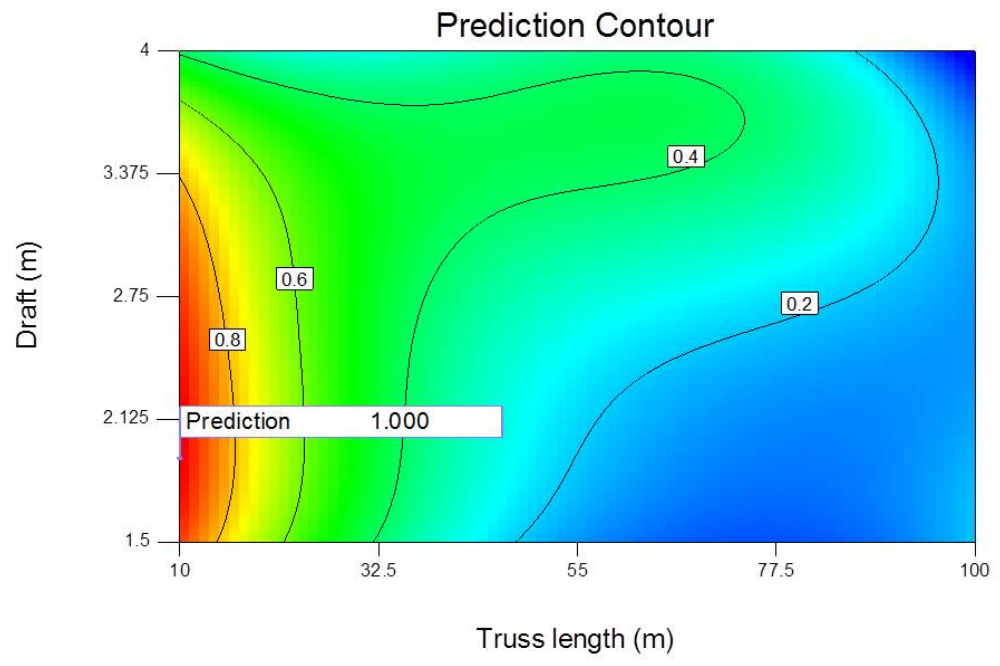

Figure 5.5: Response surface of the power capture width integration in regular waves with a wave period at 7 seconds.

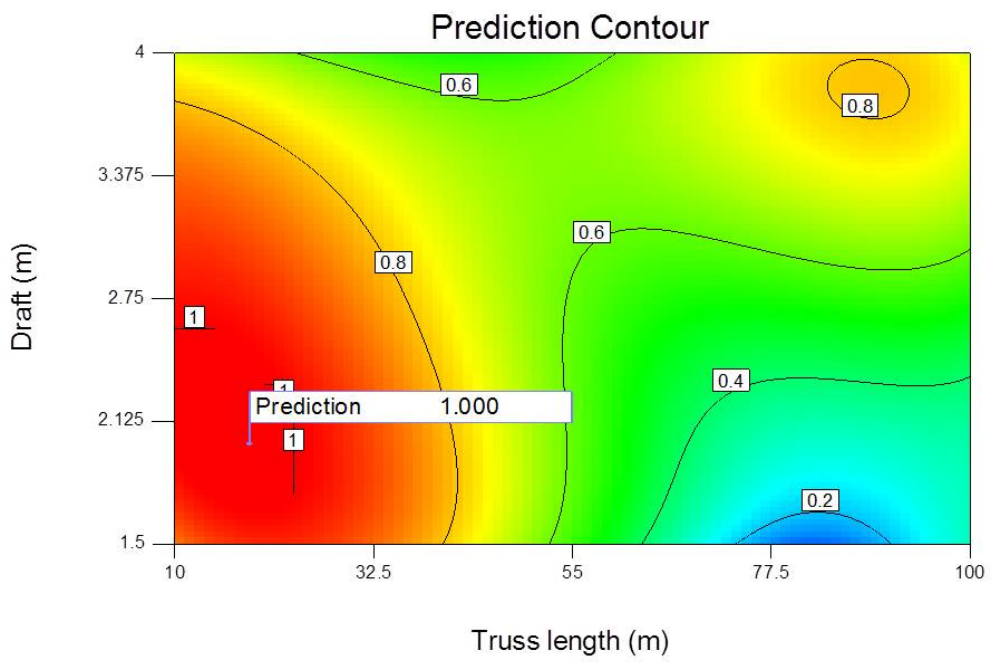

Figure 5.6: Response surface of the power capture width integration in regular waves with a wave period at 8 seconds. 
Design-Expert?Software

Factor Coding: Actual

Desirability

1.000

0.000

$X 1=A: L$

$\mathrm{X} 2=\mathrm{B}: \mathrm{T}$

Actual Factor

C: damping $=506000$

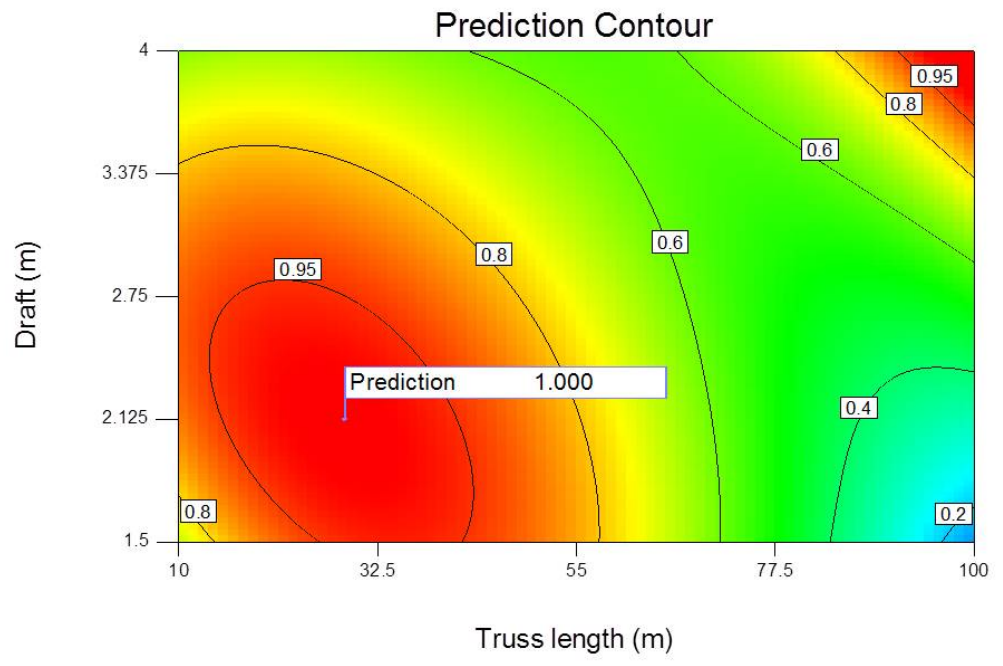

Figure 5.7: Response surface of the power capture width integration in regular waves with a wave period at 9 seconds.

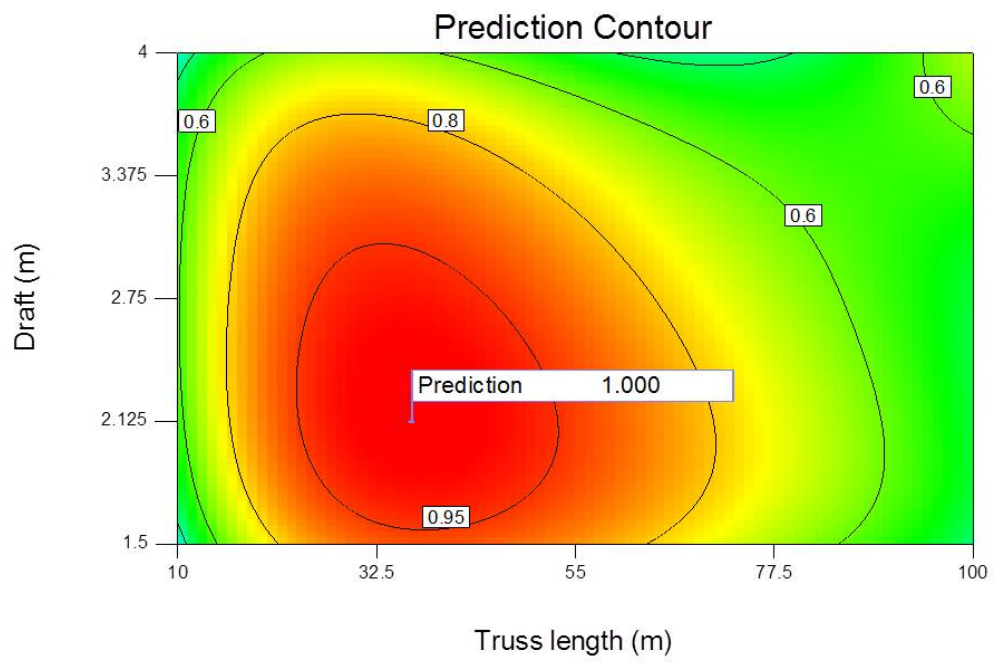

Figure 5.8: Response surface of the power capture width integration in regular waves with a wave period at 10 seconds. 
Design-Expert?Software

Factor Coding: Actual

Desirability

1.000

0.000

$X 1=A: L$

$\mathrm{X} 2=\mathrm{B}: \mathrm{T}$

Actual Factor

C: damping $=914000$

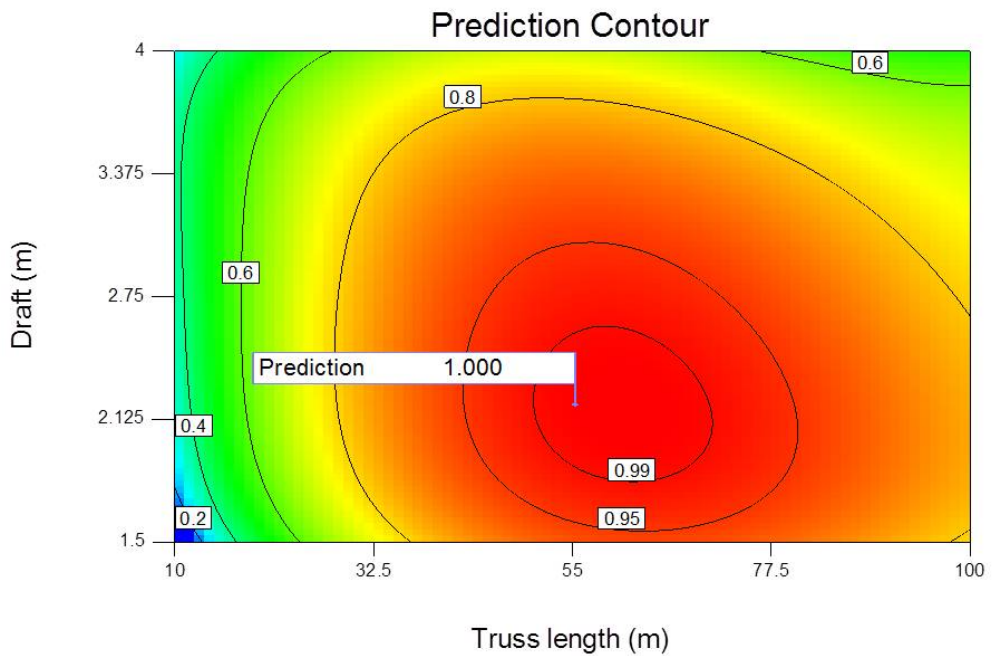

Figure 5.9: Response surface of the power capture width integration in regular waves with a wave period at 11 seconds.

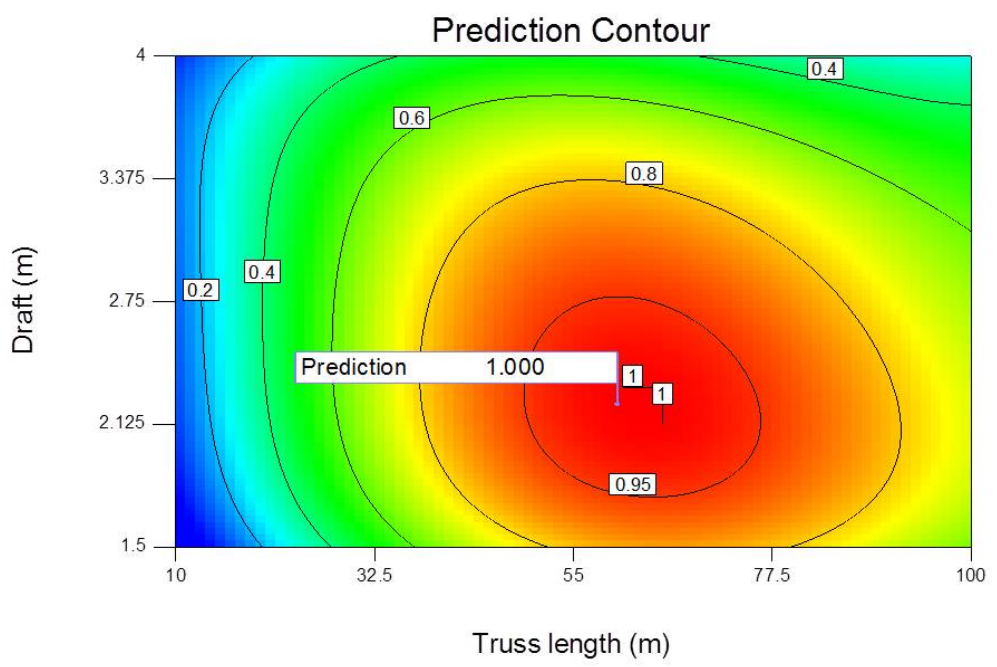

Figure 5.10: Response surface of the power capture width integration in regular waves with a wave period at 12 seconds. 
optimal combinations of truss length $L$, Draft $T$ and PTO damping $d$ were presented at each wave period.

Table 5.2: Optimization solutions at each wave period.

\begin{tabular}{cccc}
\hline Wave period $(\mathrm{s})$ & $\mathrm{L}(\mathrm{m})$ & $\mathrm{T}(\mathrm{m})$ & $\mathrm{d}(\mathrm{N} \mathrm{s} / \mathrm{m})$ \\
\hline 6 & 10 & 1.57 & $3.04 \times 10^{5}$ \\
7 & 10.2 & 1.99 & $3.05 \times 10^{5}$ \\
8 & 15.5 & 2.1 & $4.13 \times 10^{5}$ \\
9 & 28.5 & 2.13 & $5.06 \times 10^{5}$ \\
10 & 36.2 & 2.17 & $7.41 \times 10^{5}$ \\
11 & 51.4 & 2.18 & $9.15 \times 10^{5}$ \\
12 & 62.3 & 2.2 & $1.05 \times 10^{6}$ \\
\hline
\end{tabular}

It can be observed that, from 6 seconds to 12 seconds of wave period, the optimal truss length increases from 10 meters to 62 meters, and the optimal draft and damping are also increased. For a certain wave period, the best length, draft and PTO damping of SeaWEED are able to be predicted by interpolation. Figure 5.11, 5.12 and 5.13 show the optimized truss length, draft and damping values at each wave period. It is noticeable that the optimal draft of the device is not as sensitive as truss length to wave period. When the wave period is increased, the optimal draft value reaches stable at around 2.0 meters. Optimal PTO damping value rises significantly when the wave period is increased.

\subsection{Numerical Optimization at an Intended Site}

In a chosen operation site, the device was optimized according to the wave climate and the total average power is calculated according to equation 3.54. 


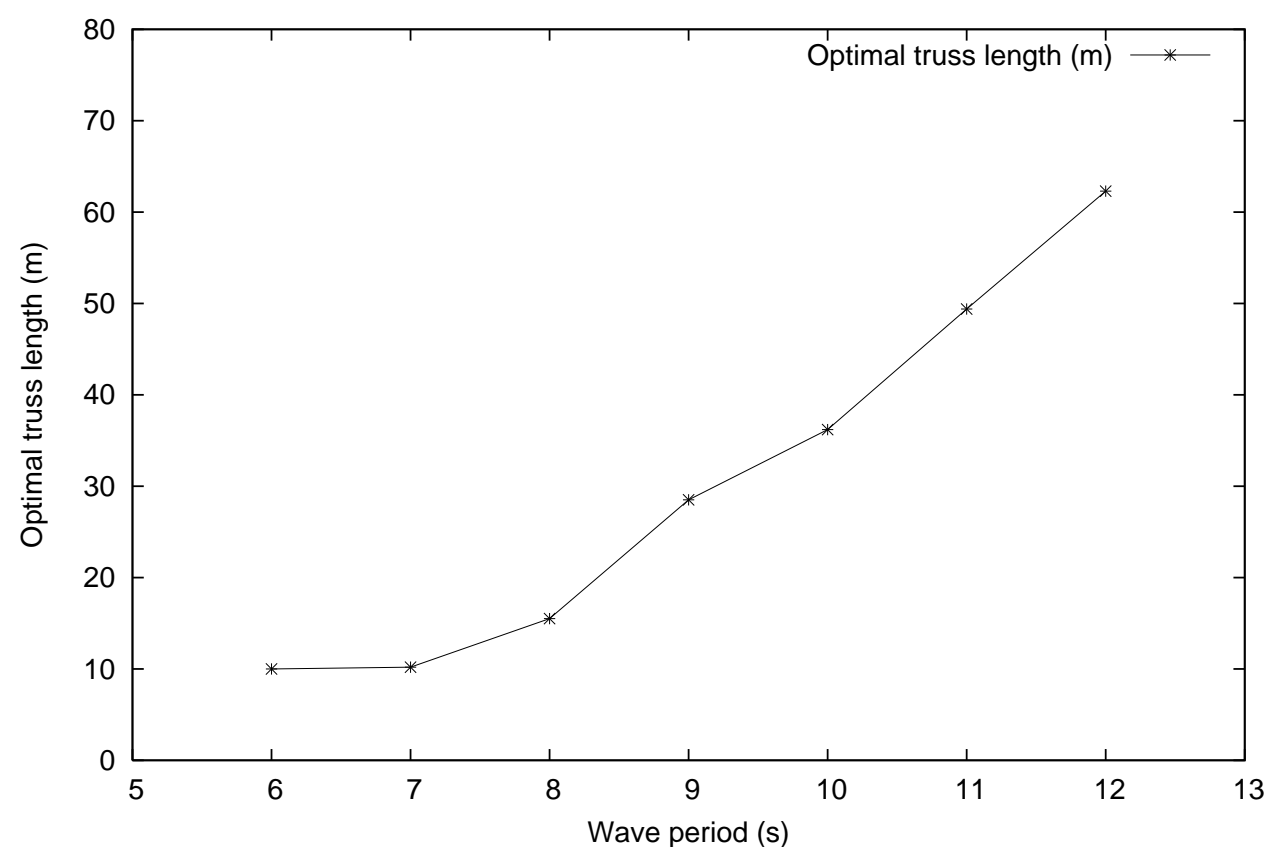

Figure 5.11: Optimal truss length at each wave period.

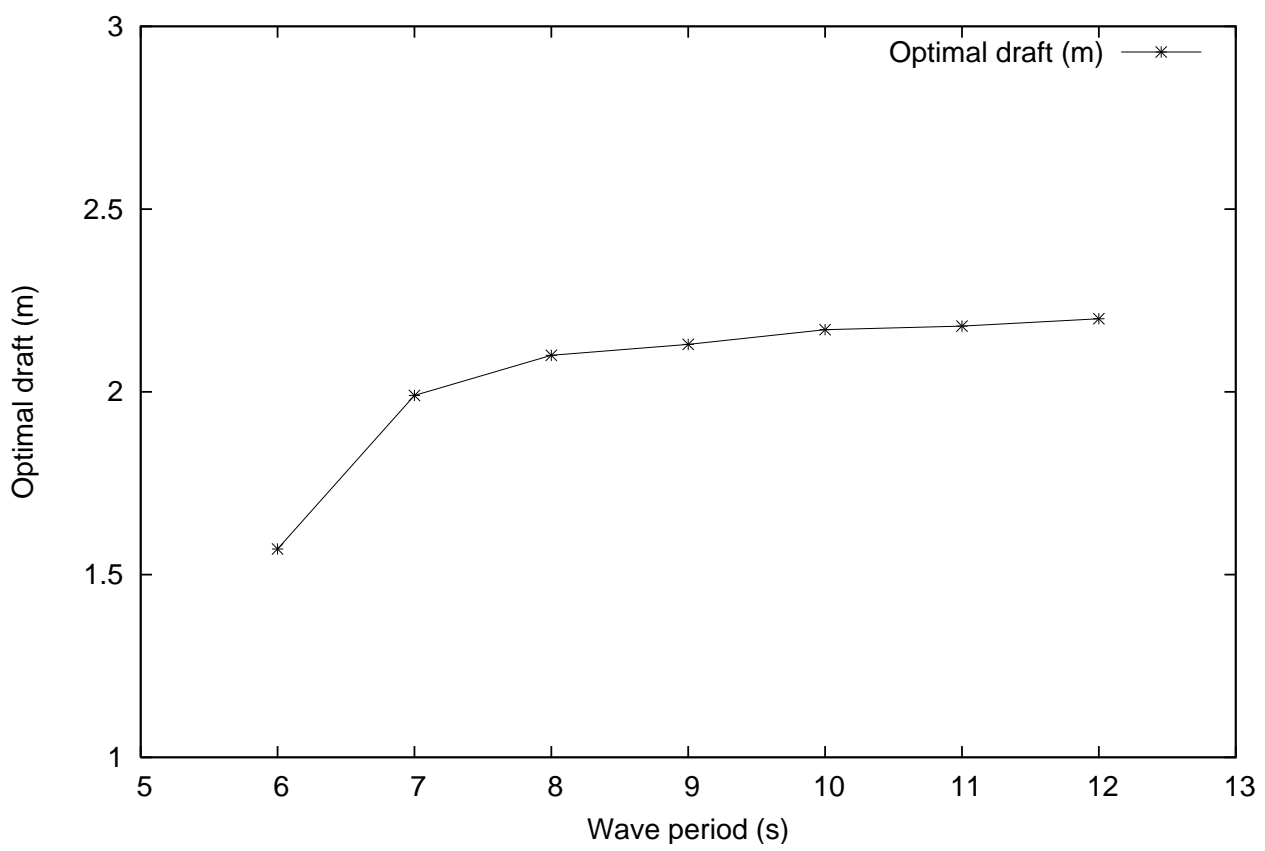

Figure 5.12: Optimal draft at each wave period. 


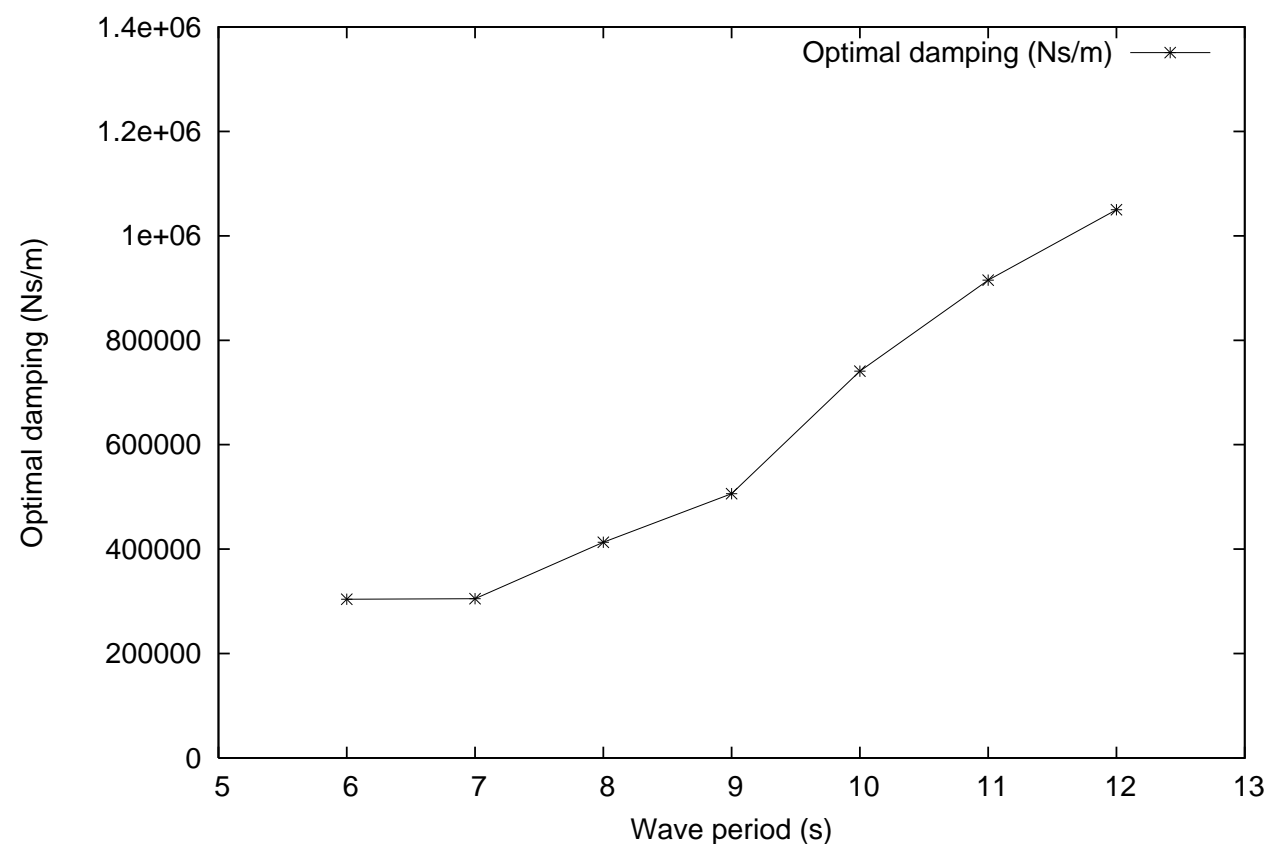

Figure 5.13: Optimal PTO damping at each wave period.

The wave climate of an intended operation site off Cork Harbour in Ireland is presented in Figure 5.14. The probability of a sea state was calculated from the percentage of the occurrence hours in a simulated year. The irregular sea states are represented by a significant wave height (Hs) and a peak period (Tp). As shown in the wave climate table that the sea states with highest probability of occurrence are around 8.5 seconds of peak periods; and mostly occurred significant wave heights are within 1.25 to 3.75 meters. The estimated annual power absorption over the year was calculated according to the given wave climate. Table 5.3 gives an overview of the optimization at an intended site. The response is the power absorption in $\mathrm{kW}$. At each wave height, the response amplitude $\xi_{i}$ was constrained by the the hinge angle $\xi_{i} / r \leq \sin 30^{\circ}$, where $r$ is the rotating arm which is the length of the corresponding hinge segment. The absorbed power for each sea state component was constrained to be less than $750 \mathrm{~kW}$ considering the capacity of the generator. 
Table 5.3: Optimization overview for an intended site.

\begin{tabular}{cccc}
\hline Variables & $\mathrm{L}(\mathrm{m})$ & $\mathrm{T}(\mathrm{m})$ & $\mathrm{d}(\mathrm{N} \cdot \mathrm{s} / \mathrm{m})$ \\
\hline Lower value & 10 & 1.5 & $10^{5}$ \\
Upper value & 100 & 4.0 & $10^{6}$ \\
Levels & 10 & 5 & 5 \\
Response & & Power absorption $\mathrm{kW}$ \\
Constraints & Joint deflection angle $\leq 30$ Degrees \\
Constraints & \multicolumn{3}{c}{ Power absorption $\leq 750 \mathrm{~kW}$} \\
\hline
\end{tabular}

\begin{tabular}{|c|c|c|c|c|c|c|c|c|c|c|c|c|c|c|}
\hline & \multicolumn{13}{|c|}{ Peak Period Tp (s) } \\
\hline & & 4.5 & 5.5 & 6.5 & 7.5 & 8.5 & 9.5 & 10.5 & 11.5 & 12.5 & 13.5 & 14.5 & 15.5 & 16.5 \\
\hline & 0.25 & $0.03 \%$ & $0.01 \%$ & $0.00 \%$ & $0.01 \%$ & $0.00 \%$ & $0.00 \%$ & $0.00 \%$ & $0.00 \%$ & $0.00 \%$ & $0.00 \%$ & $0.00 \%$ & $0.00 \%$ & $0.00 \%$ \\
\hline & 0.75 & $34 \%$ & $1.26 \%$ & $1.05 \%$ & $0.72 \%$ & $0.57 \%$ & $0.70 \%$ & $0.49 \%$ & $0.47 \%$ & $0.30 \%$ & $0.16 \%$ & $0.01 \%$ & $0.02 \%$ & $0.00 \%$ \\
\hline & 1.25 & $0.13 \%$ & $2.17 \%$ & $5.05 \%$ & $5.49 \%$ & $3.45 \%$ & $2.37 \%$ & $1.77 \%$ & $1.60 \%$ & $1.52 \%$ & $0.92 \%$ & $0.39 \%$ & $0.08 \%$ & $0.05 \%$ \\
\hline & 1.75 & $0.02 \%$ & $0.38 \%$ & $2.00 \%$ & $4.61 \%$ & $5.93 \%$ & $3.86 \%$ & $2.17 \%$ & $1.89 \%$ & $1.18 \%$ & $0.82 \%$ & $0.36 \%$ & $0.18 \%$ & $0.07 \%$ \\
\hline & 2.25 & $0.00 \%$ & $0.03 \%$ & $0.60 \%$ & $1.58 \%$ & $4.40 \%$ & $4.62 \%$ & $2.39 \%$ & $1.29 \%$ & $0.63 \%$ & $0.38 \%$ & $0.26 \%$ & $0.05 \%$ & $0.02 \%$ \\
\hline & 2.75 & $0.00 \%$ & $0.00 \%$ & $0.17 \%$ & $0.61 \%$ & $2.02 \%$ & $3.98 \%$ & $2.92 \%$ & $1.14 \%$ & $0.59 \%$ & $0.22 \%$ & $0.06 \%$ & $0.03 \%$ & $0.02 \%$ \\
\hline & 3.25 & $0.00 \%$ & $0.00 \%$ & $0.03 \%$ & $0.32 \%$ & $0.75 \%$ & $1.90 \%$ & $2.56 \%$ & $1.16 \%$ & $0.31 \%$ & $0.18 \%$ & $0.09 \%$ & $0.03 \%$ & $0.00 \%$ \\
\hline & 3.75 & $0.00 \%$ & $0.00 \%$ & $0.00 \%$ & $0.15 \%$ & $0.46 \%$ & $1.05 \%$ & $1.56 \%$ & $0.99 \%$ & $0.39 \%$ & $0.13 \%$ & $0.07 \%$ & $0.01 \%$ & $0.00 \%$ \\
\hline & 4.25 & $0.00 \%$ & $0.00 \%$ & $0.00 \%$ & $0.03 \%$ & $0.23 \%$ & $0.34 \%$ & $0.78 \%$ & $0.69 \%$ & $0.47 \%$ & $0.17 \%$ & $0.05 \%$ & $0.00 \%$ & $0.01 \%$ \\
\hline & 4.75 & $0.00 \%$ & $0.00 \%$ & $0.00 \%$ & $0.01 \%$ & $0.06 \%$ & $0.16 \%$ & $0.28 \%$ & $0.33 \%$ & $0.25 \%$ & $0.08 \%$ & $0.02 \%$ & $0.02 \%$ & $0.00 \%$ \\
\hline & 5.25 & $0.00 \%$ & $0.00 \%$ & $0.00 \%$ & $0.00 \%$ & $0.02 \%$ & $0.09 \%$ & $0.15 \%$ & $0.09 \%$ & $0.16 \%$ & $0.06 \%$ & $0.01 \%$ & $0.00 \%$ & $0.00 \%$ \\
\hline & 5.75 & $0.00 \%$ & $0.00 \%$ & $0.00 \%$ & $0.00 \%$ & $0.01 \%$ & $0.02 \%$ & $0.06 \%$ & $0.03 \%$ & $0.14 \%$ & $0.09 \%$ & $0.01 \%$ & $0.00 \%$ & $0.00 \%$ \\
\hline & 6.25 & $0.00 \%$ & $0.00 \%$ & $0.00 \%$ & $0.00 \%$ & $0.00 \%$ & $0.00 \%$ & $0.02 \%$ & $0.00 \%$ & $0.06 \%$ & $0.06 \%$ & $0.01 \%$ & $0.00 \%$ & $0.00 \%$ \\
\hline & 6.75 & $0.00 \%$ & $0.00 \%$ & $0.00 \%$ & $0.00 \%$ & $0.00 \%$ & $0.00 \%$ & $0.01 \%$ & $0.00 \%$ & $0.03 \%$ & $0.01 \%$ & $0.00 \%$ & $0.00 \%$ & $0.00 \%$ \\
\hline & 7.25 & $0.00 \%$ & $0.00 \%$ & $0.00 \%$ & $0.00 \%$ & $0.00 \%$ & $0.00 \%$ & $0.00 \%$ & $0.00 \%$ & $0.01 \%$ & $0.01 \%$ & $0.00 \%$ & $0.00 \%$ & $0.00 \%$ \\
\hline
\end{tabular}

Figure 5.14: Wave climate table for a sea location off Cork Harbour in Ireland; the datasheet comprises wave data collected from the SmartBay buoy moored in Cork Harbour (SmartBay, 2015). 
Same as the geometrical optimization process in regular waves, three factors were considered in terms of truss length, draft and the PTO damping. Two steps of optimization were conducted again. The sampling plan was designed by using the uniform design (UD) methodology, as it ensures the accuracy at a low number of runs. Eighteen combinations of truss length and draft defined 18 models. As it is known from the previous PTO optimization process that a fifth order model should be used for fitting the curve of damping effect, here for each model, only five levels of damping were needed. The response surface with the variables of the truss length and draft was fitted by a quartic model.

As shown in Figure 5.15, a circle in the middle of the response surface indicates the prediction of the annual power that exceeds the amount of $150 \mathrm{~kW}$ for a simulated year. The predicted highest annual power was $150.523 \mathrm{~kW}$ with a 32 -meter truss length and a 2.2 meter draft.

Verification tests were performed to study the accuracy of the polynomial model. The results are shown in Table 5.4. It is shown that the average error ratio for the five tests is $0.32 \%$, which means the polynomial model is reliable and valid for fitting the relationship between variables and the response.

To verify the optimal solution, the annual power of the model with a 32 -meter truss, a 2.2-meter draft, and a $4 \times 10^{5} \mathrm{~N} \cdot \mathrm{s} / \mathrm{m}$ PTO damping was computed. The calculated annual power absorption was $149.799 \mathrm{~kW}$ and the error ratio for the prediction is 0.35\%, as shown in Table 5.5.

In conclusion, the polynomial model was reliable with a low error ratio. For the intended operation site in Cork Harbour, Ireland, The predicted optimal truss length is 32 meters, the draft is 2.2 meters and the PTO damping $4 \times 10^{5} \mathrm{~N} \cdot \mathrm{s} / \mathrm{m}$. The predicted annual power absorption is around $150 \mathrm{~kW}$ in the simulated annual wave climate. 


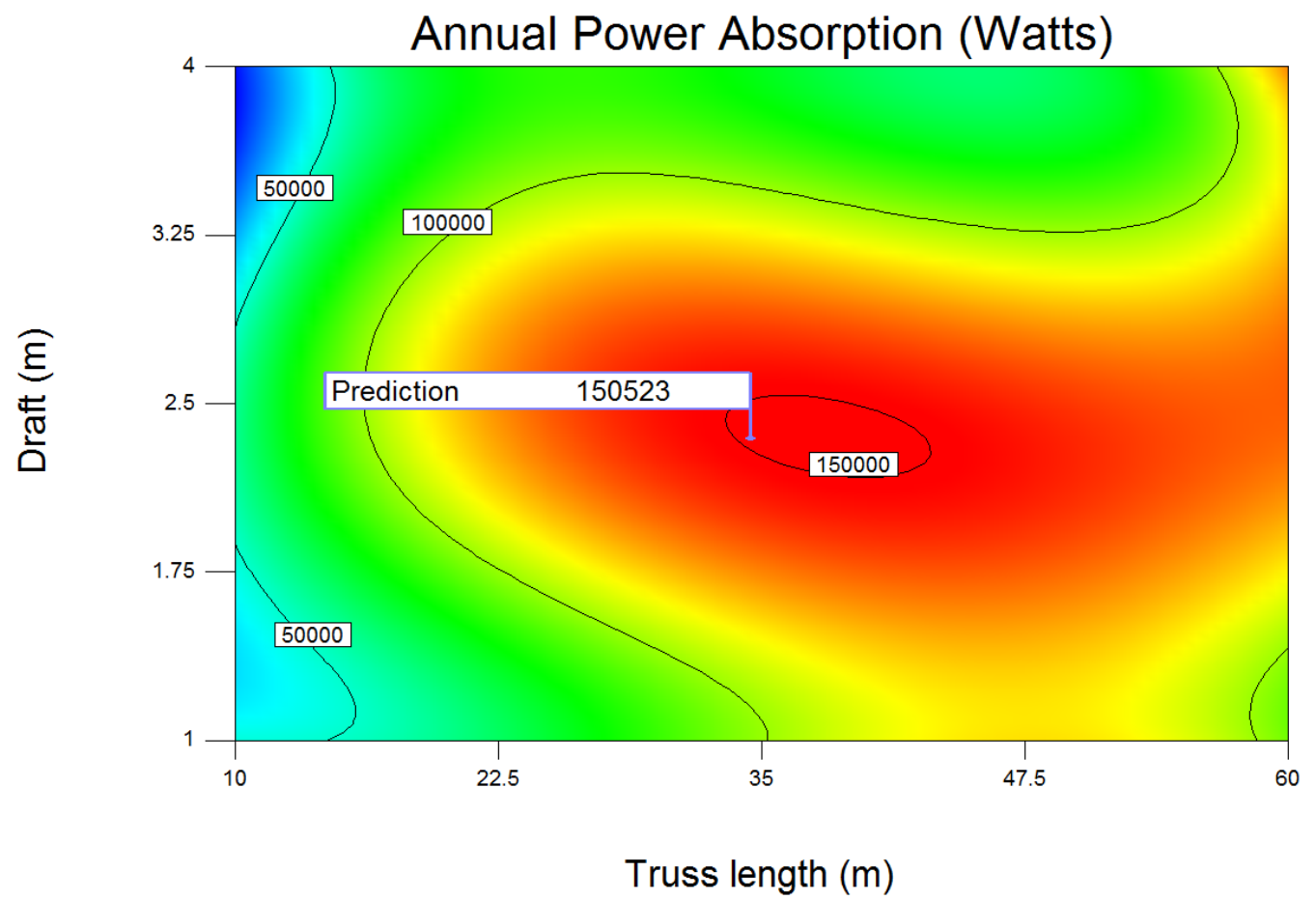

Figure 5.15: Response surface of the annual power absorption at an intended location.

Table 5.4: Verification studies on the polynomial model.

\begin{tabular}{cccccc}
\hline Variable/Response & No. 1 & No. 2 & No. 3 & No. 4 & No. 5 \\
\hline Truss length L (m) & 15 & 25 & 36 & 42 & 50 \\
Draft T (m) & 2 & 2.5 & 3.2 & 1.5 & 2.5 \\
PTO damping d (Ns/m) & 450000 & 250000 & 850000 & 950000 & 550000 \\
Predicted power (Watts) & 94983.00 & 117267.00 & 127478.00 & 99132.00 & 136075.00 \\
Calculated power (Watts) & 94703.37 & 117168.52 & 126944.00 & 98791.20 & 135474.83 \\
Error ratio & $0.30 \%$ & $0.08 \%$ & $0.42 \%$ & $0.34 \%$ & $0.44 \%$ \\
\hline Average error ratio & & & $0.32 \%$ & & \\
\hline
\end{tabular}


Table 5.5: Optimal solution, predicted annual power and computed annual power absorption.

\begin{tabular}{ccc}
\hline Variable/Response & Value & Unit \\
\hline Truss length L & 32.0 & $\mathrm{~m}$ \\
Draft T & 2.2 & $\mathrm{~m}$ \\
PTO damping d & $4 \times 10^{5}$ & $\mathrm{~N} \cdot \mathrm{s} / \mathrm{m}$ \\
Predicted annual power & 150.523 & $\mathrm{~kW}$ \\
Computed annual power & 149.992 & $\mathrm{~kW}$ \\
Error ratio & $0.35 \%$ & \\
\hline
\end{tabular}

\subsection{Comparison of the Power Absorption Perfor- mance}

\subsubsection{Comparison between the Original and Optimized Sea- WEED Models}

The truss length, draft and PTO damping of the second generation SeaWEED model were optimized due to the intended location. Comparison studies were performed on power capture width and annual power absorption between the original designed model and the optimized models. Table 5.6 presents the three models been compared in terms of the original designed model, PTO optimized model and overall optimized model. The PTO optimized model is based on the original designed parameters with only PTO damping been optimized. The overall optimized model indicates that its geometrical parameters and PTO damping were all optimized according to the intended sea site. Figure 5.16 shows the power capture width curves of the three models among 5.5 seconds to 12 seconds of wave period. The overall optimized model 
Table 5.6: Parameters of the models been compared.

\begin{tabular}{cccc}
\hline Models & Truss length $(\mathrm{m})$ & Draft $(\mathrm{m})$ & PTO damping $(\mathrm{N} \cdot \mathrm{s} / \mathrm{m})$ \\
\hline Orginal designed model & 30 & 1.5 & 700000 \\
PTO optimized model & 30 & 1.5 & 322500 \\
Overall optimized model & 32 & 2.2 & 400000 \\
\hline
\end{tabular}

has the highest capture width among 7.0 to 12 seconds. It is because that the given wave climate of the intended location has its most frequent peak period at around 8.5 seconds. Figure 5.17 shows the absorbed power at regular sea states when the wave

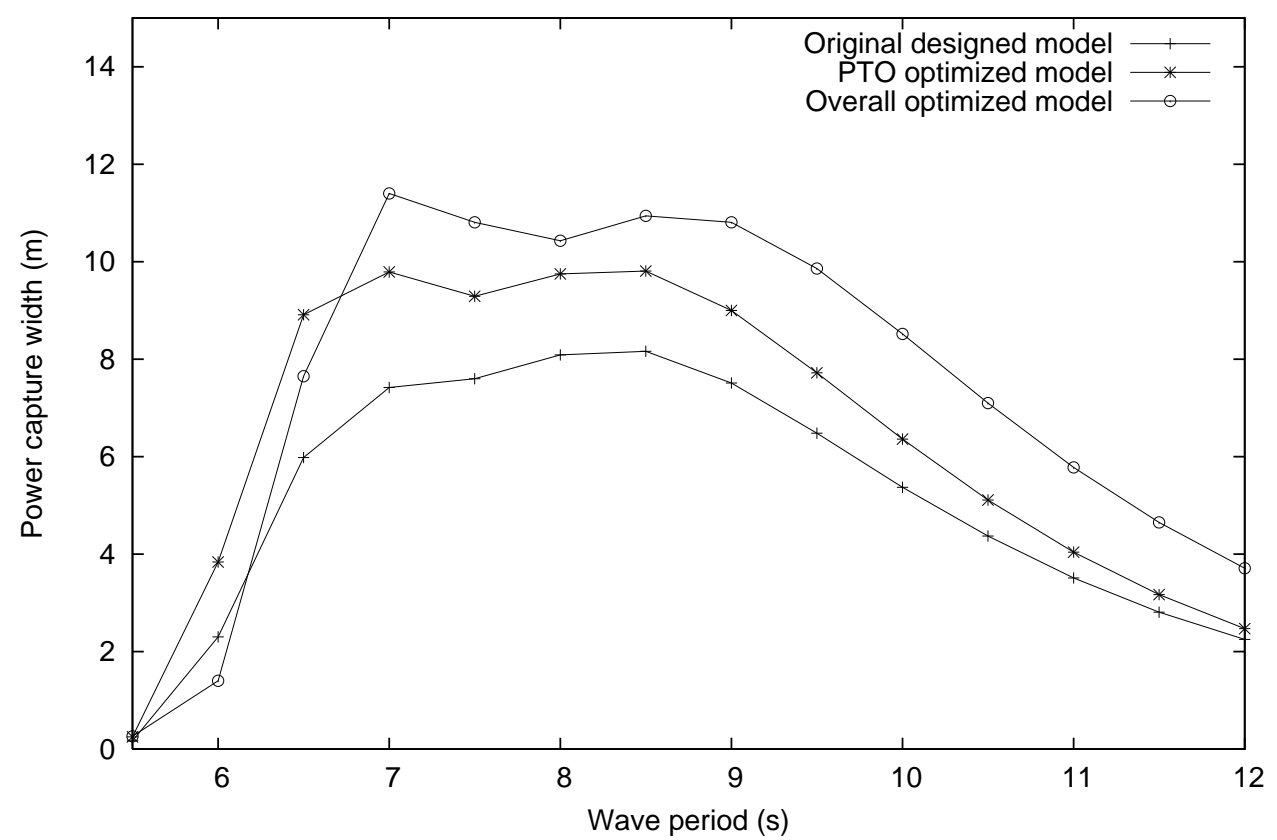

Figure 5.16: Comparison of the power capture widths between three of the second generation SeaWEED models.

height is 3 meters. The upper limit of the power absorption is $750 \mathrm{~kW}$ due to the capacity of the generator. It is shown that the overall optimized model achieve the best power absorption among the wave periods of interest. 


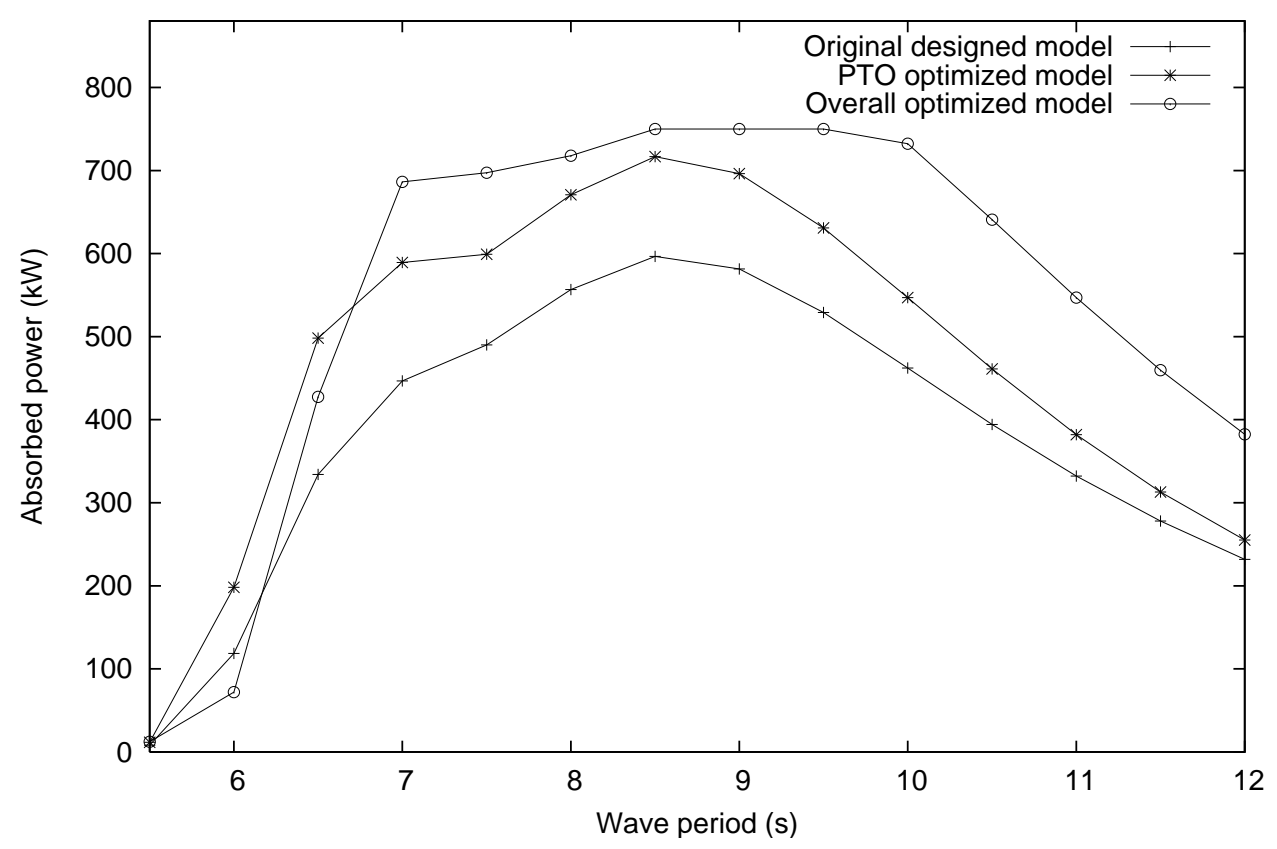

Figure 5.17: Comparison of the absorbed power between three of the second generation SeaWEED models at a 3-meter wave height.

\subsubsection{Comparison between the First and the Second Gener- ation of SeaWEED models}

The study on the first generation model in Chapter 4 shows that at the wave height of 3 meters, the results between numerical simulation and model tests matched well. A comparison between the first and the second generation of SeaWEED models is presented in Figure 5.18; both power absorption curves were computed at a 3-meter wave height. The second generation model is the overall optimized model, and the upper limit of the power absorption is $750 \mathrm{~kW}$. It is shown that among the wave periods of interest, the second generation model achieves over double power absorption than the first generation model. The optimization in geometrical parameters draft and truss length and PTO damping significantly improved the power absorption performance. It is noted that the enhancement of the interconnecting truss structure to prevent 
deformation is not able to be reflected in this numerical study.

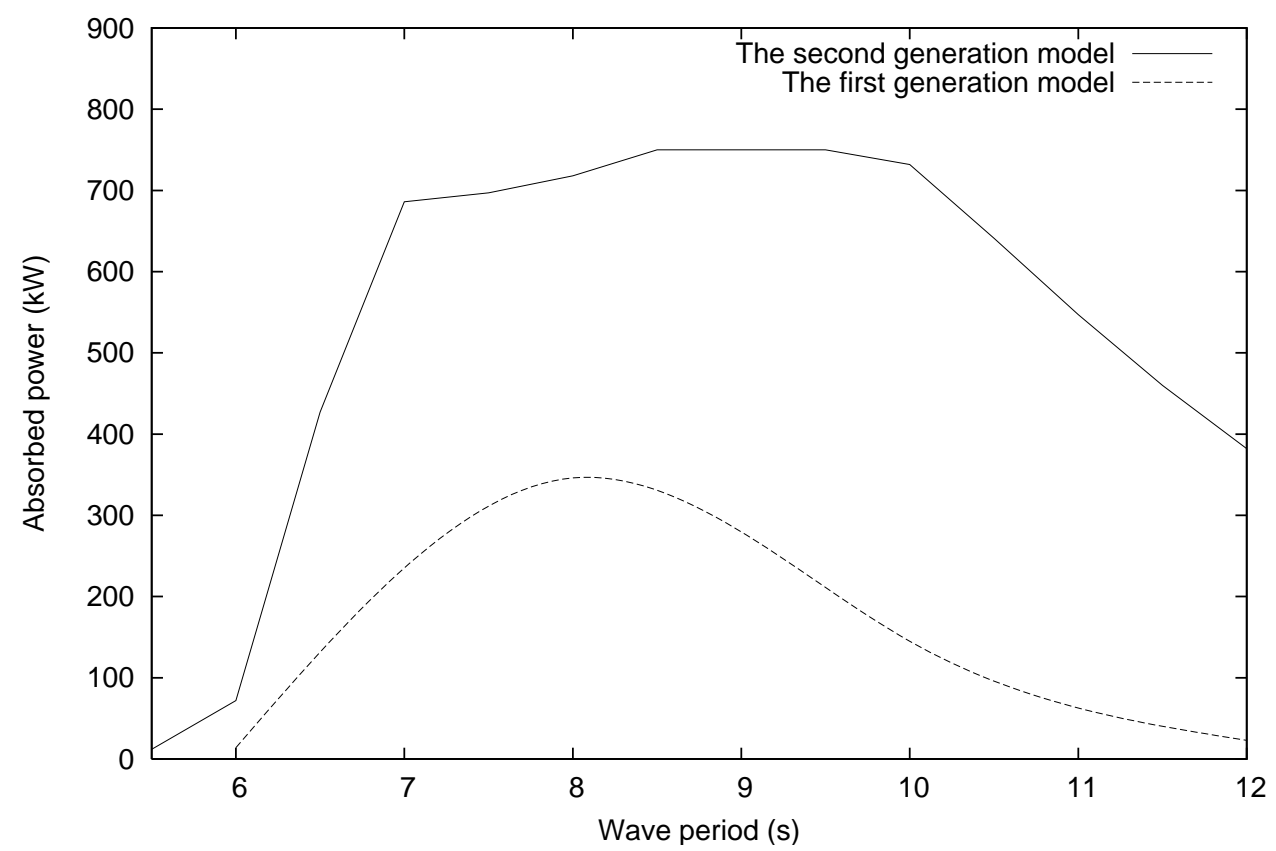

Figure 5.18: Comparison of the absorbed power between the first and the second generation SeaWEED models at a 3-meter wave height. 


\section{Chapter 6}

\section{Conclusions and Limitations}

\subsection{Conclusions}

The numerical simulation and optimization was performed for a hinged type wave energy converter called SeaWEED. SeaWEED belongs the oscillating body type of WEC. It has three members that are connected by hinged joints where the hydraulic PTO systems are located.

The system of SeaWEED was numerically simulated by a three-body hinged array with joint constraints. In hydrodynamic analysis, the PTO system was simulated as a linear-spring-damper system. Since the design of the hydraulic rams of SeaWEED does not have any spring force, the PTO stiffness was assumed as zero during the numerical simulation.

The work that have been done in this thesis is concluded as following:

- A detailed review on the wave energy converter and surrogate model optimization methodology was performed. The theories regarding a multi-body hinged array and power absorption mechanism were discussed and the formulations were derived. 
- The numerical approach was validated by comparing numerical results of the first generation SeaWEED model to experimental results of the 1:16 scale model test conducted by Grey Island Energy Incorporation. Problems found in the model test led to improvements in the hull geometry, a lower draft and a different connection structure and ultimately the second generation of SeaWEED.

- Hydrodynamic analysis and optimization was focused on the numerical model of the second generation SeaWEED. Hydrodynamic computations were performed based on the potential-flow theory by using WAMIT and combining with own pre- and post-processing codes. Convergence study was performed to determine the appropriate number of panels that is used to model the geometry of SeaWEED.

- For the optimization, the PTO damping and the geometrical parameters in terms of truss length and draft were tuned to optimize the power absorption performance in regular waves and at an intended operation site. Considering the optimal PTO damping was not an independent factor of the geometrical parameters, a two-step optimization was conducted. Firstly, the PTO damping was optimized for each model sample. Each model sample was defined by the geometrical variables of truss length and draft. Then, the optimal truss length and draft were determined under a certain wave condition.

- During the sampling plan of the simulation-based experiments, a full factorial design was used in the optimization in regular waves, which contained more runs and provided a more accurate model. For the damping effect, a sixth-order polynomial curve was generated by the software of Design-Expert for fitting the relation between damping and power absorption performance. For geometrical optimization, a quartic polynomial surface was predicted by using polynomial 
regression method to fit the relation between the geometrical variables and power capture width integration in regular waves. After the orders of the polynomial models were determined, the optimization in irregular sea states was sampled by the uniform design with fewer runs and better efficiency. The polynomial regression model that fits the response surface was verified by extra simulation experiments. The optimal solutions were predicted based on the polynomial model, and the optimal truss length, draft and PTO damping were determined for different regular wave conditions and an intended site.

\subsection{Limitations}

The numerical analysis and optimization of the second generation of SeaWEED has its limitations. Firstly, the stiffness effect of the hydraulic system was assumed to be negligible. However, in actual mechanical operation, a small effect of stiffness may exist in the bolted joints and the rams, which may lead to a smaller hinge motion and less power absorption.

Secondly, in the frequency domain simulation, potential flow theory and a linear assumption of the boundary conditions was applied to solve the body motions. However, for such slender body in high waves or rough sea states, when the boundaries are nonlinear, the linear assumption is not applicable. Therefore, CFD methods could be more accurate for solving such problems.

In conclusion, the numerical approach in this thesis was verified and validated. Further

model tests are being conducted to validate the numerical solutions for the second generation of SeaWEED model. 


\section{Bibliography}

Babarit, A., Clément, A. H., and Gilloteaux, J.-C. (2005). Optimization and timedomain simulation of the searev wave energy converter. In ASME 2005 24th International Conference on Offshore Mechanics and Arctic Engineering, pages 703-712. American Society of Mechanical Engineers.

Bergström, H. (2007). Wind resource mapping of sweden using the miuu-model. Wind Energy Report WE200\%, 1.

Bjerrum, A. (2008). The wave star energy concept. Proc. ICOE.

Box, G. E. and Wilson, K. (1951). On the experimental attainment of optimum conditions. Journal of the Royal Statistical Society. Series B (Methodological), 13(1):145.

Box, M. (1965). A new method of constrained optimization and a comparison with other methods. The Computer Journal, 8(1):42-52.

Budar, K. and Falnes, J. (1975). A resonant point absorber of ocean-wave power. Nature, 256(5517):478-479.

Campana, E. F., Liuzzi, G., Lucidi, S., Peri, D., Piccialli, V., and Pinto, A. (2009). New global optimization methods for ship design problems. Optimization and Engineering, 10(4):533-555. 
Chen, V., Tsui, K., Barton, R., and Allen, J. (2002). A review of design and modeling in computer experiments,[in:] handbook of statistics, ed. r. khattree, cr rao, vol. 22.

Clément, A., McCullen, P., Falcão, A., Fiorentino, A., Gardner, F., Hammarlund, K., Lemonis, G., Lewis, T., Nielsen, K., Petroncini, S., et al. (2002). Wave energy in europe: current status and perspectives. Renewable and sustainable energy reviews, 6(5):405-431.

Cruz, J. (2007). Ocean wave energy: current status and future prespectives. Springer Science \& Business Media.

Day, A., Babarit, A., Fontaine, A., He, Y.-P., Kraskowski, M., Murai, M., Penesis, I., Salvatore, F., and Shin, H.-K. (2015). Hydrodynamic modelling of marine renewable energy devices: A state of the art review. Ocean Engineering, 108:46-69.

Dyn, N., Levin, D., and Rippa, S. (1986). Numerical procedures for surface fitting of scattered data by radial functions. SIAM Journal on Scientific and Statistical Computing, 7(2):639-659.

Eberhart, R. and Kennedy, J. (1995). A new optimizer using particle swarm theory, proceedings of the sixth international symposium on micro machine and human science: Oct 1995.

Evans, D. (1976). A theory for wave-power absorption by oscillating bodies. Journal of Fluid Mechanics, 77(01):1-25.

Falcão, A. d. O. (2000). The shoreline owc wave power plant at the azores. In Fourth European Wave Energy Conference, Aalborg, Denmark, Dec, pages 4-6. 
Falcão, A. d. O. (2010). Wave energy utilization: A review of the technologies. Renewable and sustainable energy reviews, 14(3):899-918.

Falnes, J. (2002). Ocean waves and oscillating systems: linear interactions including wave-energy extraction. Cambridge university press.

Falnes, J. et al. (2002). Optimum control of oscillation of wave-energy converters. International Journal of Offshore and Polar Engineering, 12(02).

Fang, K.-T. (1980). The uniform design: application of number-theoretic methods in experimental design. Acta Math. Appl. Sinica, 3(4):363-372.

Fang, K.-T. and Lin, D. K. (2003). Ch. 4. uniform experimental designs and their applications in industry. Handbook of statistics, 22:131-170.

Fang, K.-T., Lin, D. K., Winker, P., and Zhang, Y. (2000). Uniform design: theory and application. Technometrics, 42(3):237-248.

Forrester, A., Sobester, A., and Keane, A. (2008). Engineering design via surrogate modelling: a practical guide. John Wiley \& Sons.

French, M., Bracewell, R., et al. (1996). The systematic design of economic wave energy converters. In The Sixth International Offshore and Polar Engineering Conference. International Society of Offshore and Polar Engineers.

Friedman, J. H. (1991). Multivariate adaptive regression splines. The annals of statistics, pages $1-67$.

Golberg, D. E. (1989). Genetic algorithms in search, optimization, and machine learning. Addion wesley, 1989.

Hansens, P. F., Haldfi, T., Knapp, W., Keller, J., Holméns, E., Holmesg, B., Thomas, G., and Rasmussen11, P. (2000). The wave dragon-now ready for test in real sea. 
Hasselmann, K., Barnett, T., Bouws, E., Carlson, H., Cartwright, D., Enke, K., Ewing, J., Gienapp, H., Hasselmann, D., Kruseman, P., et al. (1973). Measurements of wind-wave growth and swell decay during the joint north sea wave project (jonswap). Technical report, Deutches Hydrographisches Institut.

Hestenes, M. R. and Stiefel, E. (1952). Methods of conjugate gradients for solving linear systems.

Holmes, B. (2009). Tank testing of wave energy conversion systems: marine renewable energy guides. European Marine Energy Centre.

Hooke, R. and Jeeves, T. A. (1961). 'direct search' solution of numerical and statistical problems. Journal of the ACM (JACM), 8(2):212-229.

Islam, M. F. and Lye, L. (2009). Combined use of dimensional analysis and modern experimental design methodologies in hydrodynamics experiments. Ocean Engineering, 36(3):237-247.

Johnson, R. T., Montgomery, D. C., and Jones, B. (2011). An empirical study of the prediction performance of space-filling designs. International Journal of Experimental Design and Process Optimisation, 2(1):1-18.

Johnson, R. T., Montgomery, D. C., Jones, B., and Fowler, J. W. (2008). Comparing designs for computer simulation experiments. In Proceedings of the 40 th Conference on Winter Simulation, pages 463-470. Winter Simulation Conference.

Jones, B. and Johnson, R. T. (2009). Design and analysis for the gaussian process model.

Joseph, V. R., Hung, Y., and Sudjianto, A. (2008). Blind kriging: A new method for developing metamodels. Journal of mechanical design, 130(3):031102. 
Kirkpatrick, S., Gelatt, C. D., Vecchi, M. P., et al. (1983). Optimization by simulated annealing. science, 220(4598):671-680.

Kurniawan, A. and Moan, T. (2013). Optimal geometries for wave absorbers oscillating about a fixed axis. Oceanic Engineering, IEEE Journal of, 38(1):117-130.

Kushner, H. J. and Clark, D. S. (2012). Stochastic approximation methods for constrained and unconstrained systems, volume 26. Springer Science \& Business Media.

Langley, R. (1984). Random dynamic analysis of multi-body offshore structures. Ocean engineering, 11(4):381-401.

Le Mehaute, B. (1976). An introduction to hydrodynamics and water waves. Springer Verlag.

Lee, C. and Newman, J. (2013). Wamit user manual, version 7.0. WAMIT, Inc., Chestnut Hill, MA.

Lee, C.-H. and Newman, J. (2000). An assessment of hydroelasticity for very large hinged vessels. Journal of fluids and structures, 14(7):957-970.

Lekivetz, R. and Jones, B. (2014). Fast flexible space-filling designs for nonrectangular regions. Quality and Reliability Engineering International.

Margheritini, L., Vicinanza, D., and Frigaard, P. (2009). Ssg wave energy converter: Design, reliability and hydraulic performance of an innovative overtopping device. Renewable Energy, 34(5):1371-1380.

Matheron, G. (1963). Principles of geostatistics. Economic geology, 58(8):1246-1266.

McKay, M. D., Beckman, R. J., and Conover, W. J. (1979). Comparison of three methods for selecting values of input variables in the analysis of output from a computer code. Technometrics, 21(2):239-245. 
Mei, C. C. (1989). The applied dynamics of ocean surface waves, volume 1. World scientific.

Myers, R. H., Montgomery, D. C., and Anderson-Cook, C. M. (2009). Response surface methodology: process and product optimization using designed experiments, volume 705. John Wiley \& Sons.

Newman, J. (1994). Wave effects on deformable bodies. Applied Ocean Research, 16(1):47-59.

Newman, J. (1997). Wave effects on hinged bodies. part i-body motions. Technical report, technical report/http://www. wamit. com/publications. htm S.

Newman, J. N. (1977). Marine hydrodynamics. MIT press.

Noren, S. A. (1981). Plant for utilizing kinetic energy. US Patent 4,277,690.

Pizer, D., Retzler, C., and Yemm, R. (2000). The opd pelamis: experimental and numerical results from the hydrodynamic work program. In Proceedings of Fourth European wave energy conference, Aalborg, pages 227-33.

Pronzato, L. and Müller, W. G. (2012). Design of computer experiments: space filling and beyond. Statistics and Computing, 22(3):681-701.

Ross (1995). Power from Sea Waves,1st ed. United Kindom: Oxford University Press.

Rubinstein, R. Y. (1997). Optimization of computer simulation models with rare events. European Journal of Operational Research, 99(1):89-112.

Ryan, T. P. and Morgan, J. (2007). Modern experimental design. Journal of Statistical Theory and Practice, 1(3-4):501-506. 
Sacks, J., Welch, W. J., Mitchell, T. J., and Wynn, H. P. (1989). Design and analysis of computer experiments. Statistical science, pages 409-423.

Salter, S. H. (1974). Wave power. Nature, 249(5459):720-724.

Santner, T. J., Williams, B. J., and Notz, W. I. (2013). The design and analysis of computer experiments. Springer Science \& Business Media.

Simpson, T. W., Poplinski, J., Koch, P. N., and Allen, J. K. (2001). Metamodels for computer-based engineering design: survey and recommendations. Engineering with computers, 17(2):129-150.

SmartBay (2015). Smartbay ireland latest data online. https://data.smartbay.ie/.

Sun, L., Taylor, R. E., and Choo, Y. S. (2011). Responses of interconnected floating bodies. The IES Journal Part A: Civil \& Structural Engineering, 4(3):143-156.

Taghipour, R., Moan, T., et al. (2008). Efficient frequency-domain analysis of dynamic response for the multi-body wave energy converter in multi-directional wave. In The Eighteenth International Offshore and Polar Engineering Conference. International Society of Offshore and Polar Engineers.

Tahara, Y., Peri, D., Campana, E. F., and Stern, F. (2011). Single-and multiobjective design optimization of a fast multihull ship: numerical and experimental results. Journal of marine science and technology, 16(4):412-433.

Tekin, E. and Sabuncuoglu, I. (2004). Simulation optimization: A comprehensive review on theory and applications. IIE Transactions, 36(11):1067-1081.

Thorpe, T. W. (1999). A brief review of wave energy. Harwell Laboratory, Energy Technology Support Unit London, UK. 
Wang, G. G. and Shan, S. (2007). Review of metamodeling techniques in support of engineering design optimization. Journal of Mechanical Design, 129(4):370-380.

Waters, R. (2008). Energy from ocean waves: full scale experimental verification of a wave energy converter.

Waters, R., Engström, J., Isberg, J., and Leijon, M. (2009). Wave climate off the swedish west coast. Renewable Energy, 34(6):1600-1606.

Weber, J., Mouwen, F., Parish, A., and Robertson, D. (2009). Wavebob-research \& development network and tools in the context of systems engineering. In Proc. Eighth European Wave and Tidal Energy Conference, Uppsala, Sweden.

Wedderburn, R. W. (1974). Quasi-likelihood functions, generalized linear models, and the gauss - newton method. Biometrika, 61(3):439-447.

Wehausen, J. V. (1971). The motion of floating bodies. Annual review of fluid mechanics, 3(1):237-268.

Wehausen, J. V. and Laitone, E. V. (1960). Surface waves. Springer.

Yemm, R. (2008). Pelamis. Ocean wave energy. Berlin: Springer, pages 304-21.

Yemm, R., Pizer, D., Retzler, C., and Henderson, R. (2012). Pelamis: experience from concept to connection. Philosophical Transactions of the Royal Society of London A: Mathematical, Physical and Engineering Sciences, 370(1959):365-380. 